\title{
PUBLICATION ONLY
}

\section{AB01}

Unstimulated Natural Killer Cells Differentiated in Vitro from Umbilical Cord Blood CD34+ Cells Kill Patient AML Blasts in Vivo

A. Domogala ${ }^{1,2, *}$, M. Blundell ${ }^{3}$, R. Laza-Briviesca ${ }^{1}$, A. Thrasher $^{3}$, M. Lowdell ${ }^{4}$, A. Madrigal ${ }^{1,2}$, A. Saudemont ${ }^{1,2}$

${ }^{1}$ Anthony Nolan Research Institute, ${ }^{2}$ Cancer Institute, UCL, ${ }^{3}$ Institute of Child Health, UCL, ${ }^{4}$ The Royal Free Hospital, UCL, London, United Kingdom

Introduction: Natural Killer (NK) cells are cytotoxic lymphocytes of the innate immune system capable of targeting virally transformed and tumor cells without prior sensitization. They have shown to be effective at targeting malignant cells without being direct effectors of graft-versus-host disease in both a transplantation setting and as an NK cell therapy alone. There have been numerous clinical trials using NK cells as an adoptive immunotherapy in both hematological and solid malignancies. These have shown NK cell immunotherapy to be safe however efficacy has been inconsistent.

Material (or patients) and methods: In order to strive towards an effective and robust clinical therapy we hypothesize that proliferation, persistence and activation of NK cells in vivo is fundamental for the development of a clinically relevant cellular product that will be able to target tumor cells efficiently in patients. Our group has previously shown that frozen $\mathrm{CD}_{3} 4^{+}$cells isolated from umbilical cord blood (UCB) are the most favorable source of stem cells to differentiate functional NK cells in vitro. Producing large cell numbers capable of secreting high levels of IFN-g and killing leukemic cell lines in vitro. We therefore tested whether the differentiated NK cells are capable of proliferating and getting activated following further cytokine stimulation and if they are able to persist in vivo to target and eliminate leukemic cells. We also assessed if the cells could be frozen and thawed whilst still maintaining functionality to allow for the production of a readily available off-the-shelf cellular product.

Results: We found that the differentiated NK cells could respond to interleukin-2 and proliferate in vitro to the same degree as adult peripheral blood NK cells. This suggests that the cells can further respond to cytokines and proliferate once injected into the patient. The function of the differentiated cells was unaffected by cryopreservation allowing for multiple infusions at different time points of the same NK product. The differentiated NK cells could kill leukemic cells and more importantly could persist for longer and in higher numbers in vivo over other sources of NK cells, indicative of improved survival post infusion. Additionally we assessed pre-activation of the cells by priming the differentiated NK cells using CTV-1 lysate. In comparison to resting controls this consistently led to higher levels of killing of patient leukemic blasts in vitro, however this activation step was not required to observe killing of patient AML blasts in vivo. This indicates that the cells would not require prior activation before infusion resulting in a cellular therapy that was more economical and more easily translatable to the clinic.

Conclusion: In conclusion, we are able to generate and cryopreserve NK cells from UCB hematopoietic stem cells in high numbers allowing for multiple infusions that are highly cytotoxic towards tumor cells. These cells can further proliferate in response to additional cytokines and have a clear survival advantage in vivo over other sources of NK cells.
This provides the potential for the development of a robust NK cell immunotherapy which can not only prevent infection post transplantation but also target malignancies in their own right to prevent relapse.

Disclosure of Interest: None declared.

\section{AB02}

Long-Term Sustained Graft-versus-Lymphoma Effect of Donor Lymphocyte Infusion

P. Olioso ${ }^{1, *}$, S. Santarone ${ }^{1}$, A. Natale ${ }^{1}$, G. Papalinetti ${ }^{1}$, R. Giancola ${ }^{\prime}$, T. Bonfini ${ }^{1}$, P. Accorsi ${ }^{1}$, P. Di Bartolomeo ${ }^{\prime}$

${ }^{1}$ Hematology, BMT Center, O SPEDALE C IVILE, Pescara, Italy

Introduction: Allogeneic hematopoietic stem cell transplantation (SCT) is a potentially curative option for patients with lymphoproliferative disorders such as Hodgkin lymphoma $(\mathrm{HL})$ and Non-Hodgkin lymphoma (NHL). For patients who relapse or have refractory disease after SCT, the use of donor lymphocyte infusion (DLI) represent an important part of the strategy to enhance graft-versus-lymphoma effect (GvL). In this study we evaluate how DLI is able to control the disease in patients with lymphoma who relapse or have persistent disease after SCT.

Material (or patients) and methods: Between February 2001 and July 2012, 7 patients (3 males) with advanced lymphoma (high grade $\mathrm{NHL} n=5, \mathrm{HL} n=2$ ) previously treated with SCT received DLI as treatment for progressive or relapsed disease. The median age was 35 (21-52) years at time of SCT. Three of them underwent previous autologous SCT. All patients were transplanted with PBSC, 6 from HLA identical donor and 1 from an unrelated one. Conditioning was myeloablative in 1 and reduced intensity in 6. GvHD prophylaxis included Cyclosporine and short course methotrexate in all patients.

Results: No patient developed DLI-related aplasia or acute GvHD after DLI. Limited chronic GvHD was observed in only 2 patients. Three patients had no response to DLI and died for progression of their lymphoma. Four patients had sustained complete response with persistent and continued disappearance of the lymphoma. The cumulative response rates after DLI to treat relapse or persistent disease after SCT were $3 / 4$ (75\%) and $1 / 3(33 \%)$, respectively. No correlation was observed between $\mathrm{CD} 3+$ cell dose infused and disease response. Both patients who developed DLI-related chronic GvHD had complete response. All four patients who achieved complete response after DLI are still alive and disease-free after a median follow-up of 11 (9-12) years.

Conclusion: These data support the existence of a clinically significant GvL effect in high-grade $\mathrm{NHL}$ and aggressive $\mathrm{HL}$ suggesting that DLI is an effective treatment able to induce long-term remission without severe GvHD in patients with very poor prognosis. A DLI program should always be considered for patients with lymphoma who show persistent disease or relapse after SCT.

Disclosure of Interest: None declared. 
AB03

Human platelet lysate pathogen reduced through additive-free short-wave UV light irradiation retains its optimal efficacy for the expansion of human bone marrow mesenchymal stem cells

S. Viau', L. Chabrand', S. Eap ${ }^{1}$, F. Goudaliez', C. Sumian², B. Delorme ${ }^{1, *}$

${ }^{1}$ Biotherapy Division, Macopharma, Mouvaux, ${ }^{2}$ Transfusion Division, Macopharma, Tourcoing, France

Introduction: We recently developed and characterized a standardized and clinical grade human Platelet Lysate (hPL) that constitutes an advantageous substitute for fetal bovine serum for human mesenchymal stem cell (hMSC) expansion required in cell therapy procedures, avoiding xenogenic risks (virological and immunological) and ethical issue. Because of the progressive use of pathogen reduced (PR) labile blood components, we evaluated the impact of the novel procedure THERAFLEX UV-Platelets for pathogen reduction on hPL quality (growth factors content) and efficacy (as a medium supplement for hMSC expansion). This technology is based on short-wave ultraviolet light (UV-C) and has the main advantage not to need the addition of any photosensitizing additive compounds (that might secondary interfere with hMSCs).

Material (or patients) and methods: We applied THERAFLEX UV-Platelets procedure on fresh platelet concentrates (PCS) suspended in platelet additive solution and prepared $\mathrm{hPL}$ from these treated PCs. We compared the quality and efficacy of PR$\mathrm{hPL}$ with the corresponding non-PR ones.

Results: We showed no impact on the content in 5 cytokines tested (EGF, bFGF, PDGF-AB, VEGF and IGF) and a significant decrease in TGF-b1 $(-21 \%, n=16, P<0.01)$. We performed large scale culture of hMSCs during 3 passages and showed that $\mathrm{hPL}$ or $\mathrm{PR}-\mathrm{hPL}$ at $8 \%$ triggered comparable hMSC proliferation than FBS at $10 \%$ plus bFGF $(n=3)$. Moreover, after proliferation of hMSCs in hPL or PR-hPL containing medium, their profile of membrane marker expression, their clonogenic potential and immunosuppressive properties were maintained, in comparison with hMSCs cultured in FBS conditions. The potential to differentiate in adipogenic and osteogenic lineages of hMSCs cultured in parallel in the 3 conditions remained also identical.

Conclusion: In conclusion, we demonstrated the feasibility to use UV-C treatment to subsequently obtain pathogen reduced $\mathrm{hPL}$, while preserving its optimal quality and efficacy for hMSC expansion for cell therapy applications.

Disclosure of Interest: S. Viau Employee of: Macopharma, L. Chabrand Employee of: Macopharma, S. Eap Employee of: Macopharma, F. Goudaliez Employee of: Macopharma, C. Sumian Employee of: Macopharma, B. Delorme Employee of: Macopharma.

АB04

Effect of refreezing and long term storage of hematopoietic stem cells regarding cell count, viability and apoptosis

B. Omazic ${ }^{1, *}$, M. Larsson ${ }^{1}$, J. Makivic ${ }^{1}$

${ }^{1}$ Clincal Immunology, Karolinska University hospital Huddinge, Stockholm, Sweden

Introduction: In patients with myeloma, it might be necessary to perform more than one autologous hematopoietic stem cell transplantations (ASCT). At our centre, we have been have asked to refreeze parts of autologous hematopoietic stem cells (HSC) units with high cell numbers, that already have been stored for several years. The idea is to use the refrozen part of the cells if there is a need for re-transplantation. This raises the question of the viability of the graft.

Material (or patients) and methods: The aim of the study was to analyze the effect of refreezing on HSC regarding the amount of CD34+ cells, cell viability, apoptosis and regeneration ability. HSC products from ten deceased patients, stored for more than 8 years, were analyzed regarding the amount of CD34+ cells, cell viability measured with 7-AAD and apoptosis measured with Annexin V. We used flowcytometry for the analysis. The regeneration ability of HSC was analyzed with colony forming units.

Results: Refreezing of HSC product, did not affect the amount of CD34+ cells significantly. However a significant decrease in cell viability, with a mean of $20 \%$, was seen. The number of apoptotic cells was significantly increased with $26 \%$. The regenerative ability, measured as colony forming units, decreased significantly with a mean of $31 \%$

Conclusion: It can be concluded, that refreezing of HSC product decreases the number of viable CD34+ cells with approximately $30 \%$.

Disclosure of Interest: None declared.

\section{AB06}

Differences in Umbilical Cord Blood Units collected during Cesarean section or after Vaginal deliveries: A Single Center Analysis of the Leuven Cord Blood Bank

F. Sinap ${ }^{1, *}$, T. Blevi ${ }^{1}$, Y. Vanbriel ${ }^{1}$, D. Reggers ${ }^{1}$, B. Janssens ${ }^{1}$, J. Klykens ${ }^{1}$, M. Boogaerts ${ }^{1}, T$. Devos ${ }^{1}, H$. Schoemans ${ }^{1}$

${ }^{1}$ Leuven Cord Blood Bank, UZ Leuven Campus Gasthuisberg, Leuven, Belgium

Introduction: Umbilical cord blood (UCB) is largely employed as an alternative source of hematopoietic stem cells in the treatment of hematological diseases. One of the limitations to use UCB is the relative low content of hematopoietic stem cells. The main parameters used in umbilical cord blood banks include total nucleated cell (TNC) count, percentage of CD34+ cells and collection volume. The Leuven Cord Blood Bank (LNBB) collects units from a network of maternity wards since 1997 using the 'in utero' collection method both for vaginal deliveries and cesarean sections. Since 2015 the LNBB has updated the UCB databank to register mode of delivery of every UCB collection.

Material (or patients) and methods: This analysis compares UCB obtained from 2855 vaginal deliveries and 146 caesarean sections $(01 / 2015-11 / 2015)$. The volume of the collected cord blood was compared as well as quality parameters such as cell numbers (TNC) before processing and CD34+ cell content post-processing, by an independent Samples T-test. The level of significance was set for a $p$-value $<0.05$

Results: Mean volume of UCB collected after cesarean section was significantly higher compared to UCB volumes collected after vaginal deliveries $(P<0.0001)$ (table 1$)$. No statistically

Table 1. UCB data from Cesarean and Vaginal deliveries

\begin{tabular}{lccc}
\hline Parameter & Cesarean & Vaginal & P value \\
\hline Collected & $87.34 \pm 2.9$, & $\begin{array}{c}70.29 \pm 0.6, \\
n=2855\end{array}$ & $<0.0001$ \\
volume UCB & $n=146$ & $n$ & \\
$(\mathrm{ml})$ & & & \\
TNC count & $1398 \pm 69.96$, & $1502 \pm 18.38$, & 0.135 \\
$\left(\mathrm{x} 10^{6}\right)$ & $n=70$ & $n=914$ & \\
CD34+ $\left(\times 10^{6}\right)$ & $\begin{array}{c}5.682 \pm 0.8263, \\
\end{array}$ & $\begin{array}{c}5.237 \pm 0.1929, \\
n=16\end{array}$ & 0.583 \\
& & $n=265$ & \\
\hline
\end{tabular}

Results are expressed as mean $\pm \mathrm{SD}$.

significant difference between quality markers such as cell count and CD34+ count (table 1) could be detected.

Conclusion: Cord blood collection after cesarean route of delivery showed a larger collection volume than vaginal delivery. A possible explanation could be that, as the baby is placed above the placenta after the cesarean section, clamping the cord above the placenta possibly causes a downward flow of blood into the umbilical cord, which may result in a greater volume of UCB collected (Nunes \& Zandavalli, 2015). Despite the difference in collected volume, UCB units after cesarean sections seem to contain similar progenitor content compared to vaginal deliveries as 
described in the literature (Askari et al, 2005). In utero UCB collections after cesarean sections are feasible, but further studies are needed to investigate the impact of the type of delivery on quality of the cord blood units.

References: Nunes R., Zandavalli F. Association between maternal and fetal factors and quality of cord blood as source of stem cells. Rev Bras Hematol Hemater. 2015;37(1):38-42.

Akari et al. Impact of donor-and collection-related variables on product quality in ex utero cord blood banking. Transfusion 2005; Volume 45:189-194.

Disclosure of Interest: None declared.

\section{AB07}

Ten years experience of autologous and allogeneic stem cell transplantation - a single centre Sofia, Bulgaria (CIC 859)

G. Arnaudovi, , P. Ganeva ${ }^{1}$, Y. Petrov' ${ }^{1}$, G. Mihaylov ${ }^{1}$ on behalf of Martin Donchev, Kalina Ignatova, Vera Stoeva, Svetla Ivanova, Margarita Guenova, Georgui Balatzenko, Lidia Gartcheva, Antoaneta Mihova

${ }^{1}$ National Hospital for Active Treatment of Hematological Diseases, Sofia, Bulgaria

Introduction: The hematopoietic stem cell transplantation (HSCT) has become a standart in the treatment of certain hematological malignencies, solid tumors, autoimmune diseases, immune disorders etc. Data from the world and European records show yearly increase in the number of transplanted patients, periodically updating new indications based on the analyzed results from the transplantation (autologous and allogeneic).

Material (or patients) and methods: The BMT unit (CIC 859) was established in 2004. We retrospectively analyzed data from 482 patients over 10 years (2004- DEC 2014): 419 autologous, 63 allogeneic (2010-2014). The median age of patients in the autologous group is 43 years (20-67), and 35,8 (20-59) in the allogeneic group. The number of transplanted patients in years was as follows: $2004(\mathrm{~N}=10)$ $2005(\mathrm{~N}=23) ; 2006(\mathrm{~N}=18) ; 2009(\mathrm{~N}=34) ; 2010(\mathrm{~N}=59), 2011$ $(\mathrm{N}=73), 2012(\mathrm{~N}=83) ; 2013(\mathrm{~N}=94) ; 2014(\mathrm{~N}=87)$. In the autotransplanted group the large proportion of patients took those with Multiple Myeloma $(N=171)$, followed by Hodgkin's Disease $(\mathrm{N}=114)$ and $\mathrm{NHL}(\mathrm{N}=108)$, solid tumors $(\mathrm{N}=4)$ and AML $(N=8)$. Allogeneic stem cell transplantation was performed in patients with Aplastic Anemia $(\mathrm{N}=5), \mathrm{AML}(\mathrm{N}=32)$, ALL $(N=17), C M L(N=1), N H L(N=1)$, Hodgkin's Disease $(\mathrm{N}=1)$, Multiple Myeloma $(\mathrm{N}=3)$, MDS $(\mathrm{N}=1), \mathrm{CL}(\mathrm{N}=1)$ plasma cell leukemia $(\mathrm{N}=1)$. Depending on availability of donor 35 patients were transplanted with Related Donor (RD), 28 patients with Match Unrelated Donor or Mismatch Unrelated Donor. The use of conditioning regimens was as follows - Myeloablative regimen $(\mathrm{N}=46)$, Reduce intensity conditioning $(\mathrm{N}=17)$.

Results: In this overview were discussed Overall survival (OS), Disease free survival (DFS) in different groups, the factors for successful control of the underlying disease, the risks and therapy related complications. In the allogeneic group are considered causes of early death: transplant related mortality $(\mathrm{N}=4)$, death from relapse $(\mathrm{N}=5)$, death from GvHD ( $N=14)$.

Conclusion: The results showed an increase number of both autologous and allogeneic transplants - a global trend. The low TRM proves that this high-tech procedure leads to increase OS in some patients and increase their chances for a long DFS.

Disclosure of Interest: None declared.

\section{AB08}

Pneumomediastinum Case Secondary to Pneumocystis Jirovecii Infection as Late Complication in an Allogeneic Hematopoietic Stem Cell Tranplant Patient

G. Akyol l,* E. Ermiş Turak ${ }^{1}$, E. Yıldızhan ${ }^{1}$, K. Demir ${ }^{1}$, M. Çetin ${ }^{1}$, L. Kaynar

${ }^{1}$ Hematology, Erciyes Univercity, Kayseri, Turkey

Introduction: Pneumocystis jirovecii pneumonia (PJP) is important cause of mortality and morbidity in immunocompromised patients with especially acquired immunodeficiency syndrome (AIDS),and others like hematopoietic stem cell tranplantion or hematologic malignancies. Even though pneumothorax is an expected complication during PJP, pneumomediastinum is quite rare. We report a case of spontaneous pneumomediastinum during treatment of late onset P.jirovecii pneumonia in an allogeneic hematopoietic stem cell tranplant recipient.

Material (or patients) and methods: Case Report

23-year-old male patient who underwent allogeneic hematopoietic stem cell tranplantation sixteen months ago, admitted to bone marrow transplantation clinic for shortness of breath. There was widespread rhonchi in bilateral lungs. Patient had no fever with arterial blood gase oxygen level measured as $\mathrm{SaO} 2$ 89\%. Computed tomography showed significant consolidation lung areas of in both upper lobe (Figure 1). Pneumocystis jirovecii pneumonia diagnosis was considered so that high TMP-SMX doses intravenously with $1 \mathrm{mg} / \mathrm{kg}$ methylprednisolone was started. Piperacillin-tazobactam added to therapy as bacterial pneumonia can not be ruled out. In the fifth day of treatment, arterial blood gas $\mathrm{SaO} 2$ was $95 \%$. On control chest CT obtained for crepitus in the bilateral supraclavicular region and pain on anterior chest wall revealed gas density surrounding both hilar and mediastinal structures with the extension of the right supraclavicular region. This view was regarded as pneumomediastinum ten days after hospitalization (Figure 2). There was no reason as a bronchoscopic procedure or trauma that can cause images in history. Piperacillin-tazobactam therapy was discontinued. TMP-SMX continued intravenously. Control CT taken 1 month later, pnönomediastinum appearance resolved completely (Figure 3).

Results: Discussion

Conclusion: Most frequent causes of pneumomediastinum are barotrauma, asthma which led to increased intrathoracic pressure increase, cavitation forming pneumonia or invasive

[AB08]

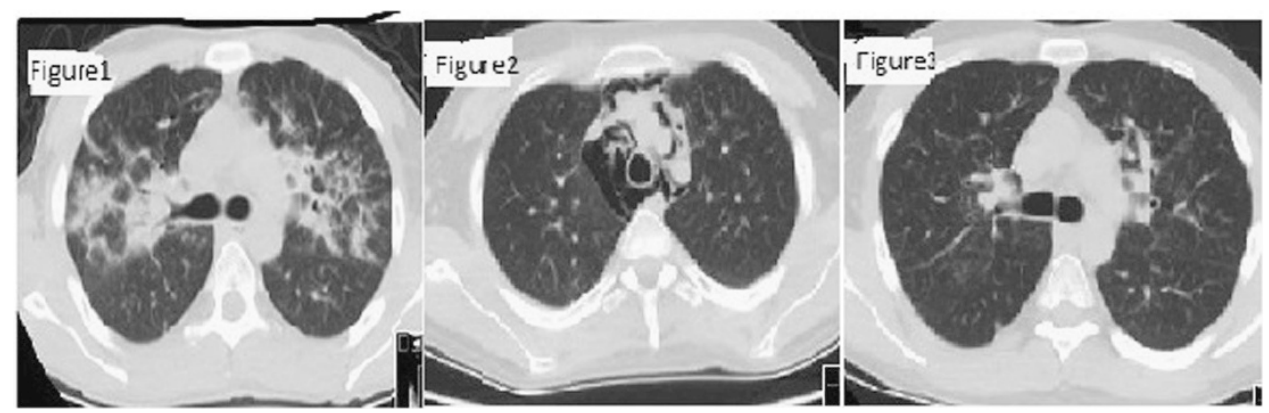


procedures etc. Spontaneous pneumothorax is often reported to be $35 \%$ during the PJP, however pneumomediastinum is often mentioned in the literature as rare cases. And these cases usually resolve within 1-2 weeks. If infection is considered, antibiotherapy must be started immediately and the protection of patients barotrauma are crucial.

Disclosure of Interest: None declared.

AB09

Anti-proliferative effect of natural killer subsets on hematopoietic stem and progenitor cells

J. Riewaldt ${ }^{1,2, *}$, S. Sayed ${ }^{1,3}$, T. Tonn ${ }^{1,2,3}$

${ }^{1}$ Experimental Transfusion Medicine, German Red Cross Blood Donor Service North East, ${ }^{2}$ Transfusion Medicine, Medical Faculty, Technical University Dresden, ${ }^{3}$ Center for Regenerative Therapies Dresden, Dresden, Germany

Introduction: Human hematopoietic stem and progenitor cells (HSPCs) reside in the bone marrow niche within a complex cellular network including mesenchymal stromal cells and various immune cells such as osteoblasts, T lymphocytes and megakaryocytes (Boulais \& Frenette, 2015). In addition, the innate immune system - with natural killer (NK) cells as one of its major components - is present in the bone marrow niche, although it is currently not clear whether and how it may contribute to HSPC homeostasis. Here, we will present data that show negative regulation of hematopoiesis by NK cell subsets in vitro.

Material (or patients) and methods: Human mononuclear cells (MNCs) were isolated from either peripheral blood (for NK cell isolation), G-CSF-mobilized peripheral blood or bone marrow (for HSPC isolation) by density gradient centrifugation after obtaining informed consent of the donors. The study was approved by the local institutional review board. CD34 ${ }^{+}$HSPCs were enriched from MNCs using a magnetic activated cell sorting (MACS) approach, while different subsets of NK cells were obtained by either MACS technology or fluorescenceactivated cell sorting (FACS). HSPCs and NK cell subsets were co-cultured in vitro in semi-solid medium to assess colony forming unit capacities of HSPCs.

Results: Co-cultivation of HSPCs with purified $\mathrm{CD} 56^{+}$NK cells resulted in drastically diminished colony formation after two weeks, as compared to cultivation of HSPCs alone. Although both myeloid and erythroid progenitor numbers were found to be reduced by the presence of NK cells, erythroid colonies appeared to be stronger negatively affected than myeloid colonies. Co-culturing of different NK subpopulations FACSpurified based on surface antigen expression appeared to have differential impact on HSPC differentiation/ colony formation capacity.

Conclusion: Based on our data we conclude that the innate immune system contributes to control of HSPC fate and behavior by suppressing HSPCs differentiation and growth in vitro through specific NK cell subsets. Further studies to dissect specific interaction mechanisms are required.

References: Boulais P.E. and P.S. Frenette: Making sense of hematopoietic stem cell niches. Blood, Blood. 2015 Apr 23;125 (17):2621-9.

Disclosure of Interest: None declared.

AB10

Impact of CD34+ stem cell dose on early mortality after allogeneic hematopoietic stem cell transplantation K. Neumann ${ }^{1, *}$, S. Lange ${ }^{1}$, B. Kragl ${ }^{1}$, Ä. Glass ${ }^{2}$, C. Grosse-Thie ${ }^{1}$, C. Wittke ${ }^{1}$, S. Freitag ${ }^{1}$, L. Henze ${ }^{1}$, H.-D. Kleine ${ }^{3}$, C. Junghanss ${ }^{7}$ ${ }^{1}$ Hematology/Oncology/Palliative Care, ${ }^{2}$ Institute for Biostatistics and Informatics in Medicine and Ageing Research, University of Rostock, ${ }^{3}$ Seracell Stammzelltechnologie GmbH, Rostock, Germany

Introduction: The outcome of allogeneic hematopoietic stem cell transplantation (HSCT) depends on a variety of patient-, transplant-, and donor-related factors. Our retrospective study aimed at the evaluation of the potential risk factor CD34+ stem cell dose on early mortality after allogeneic HSCT.

Material (or patients) and methods: Data sets of 436 consecutive patients who underwent allogeneic HSCT at a single center (University of Rostock) from 1998 to 10/2015 were analysed regarding their early mortality rates $(d+30$, $d+60$ ). Variables included in Cox regression analysis were viable CD34+ cell dose, recipient sex, age, body mass index (BMI), diagnoses, conditioning intensity, stem cell source, posttransplant immunosuppression and donor type.

Results: Median patient age at time of HSCT was 49 years (15-76 years) with the majority of patients (59\%) being male. Median transplanted CD34+ cell dose was $4.4 \times 10^{6} / \mathrm{kg}$ $\left(3.7 \times 10^{5}-2.2 \times 10^{7} / \mathrm{kg}\right)$. Sixty-one patients $(14 \%)$ received cell doses $<2.0 \times 10^{6}$ and 10 patients $(2.3 \%)<1.0 \times 10^{6}$ CD34+ cells $/ \mathrm{kg}$. For further analyses the cohort was divided in groups by cell dose (quartiles): $<2.71 \times 10^{6}\left(<25^{\text {th }}\right.$ percentile; group 1), $2.71-5.85 \times 10^{6}\left(25^{\text {th }}-75^{\text {th }}\right.$ percentile; group 2$) ;>5.85 \times 10^{6} / \mathrm{kg}$ ( $>75^{\text {th }}$ percentile; group 3 ).

Transplant indications were acute myeloid leukemia/myelodysplastic syndrome (45\%), acute lymphoid leukemia (8\%), chronic myeloid leukemia (10\%), non-hodgkin lymphoma $(15 \%)$, chronic lymphoid leukemia (4\%), hodgkin disease (1\%), multiple myeloma (8\%) and other diseases (9\%). Preparative regimen comprised standard intensity conditioning (28\%) and reduced intensity or nonmyeloablative conditioning (72\%). Most patients underwent HSCT from matched unrelated donors (54\%) or matched related donors (33\%). Grafts originated from bone marrow $(n=72)$ or peripheral blood $(n=364)$ contained a median CD34+ cell dose of $2.0 \times 10^{6} / \mathrm{kg}$ and $5.0 \times 10^{6} / \mathrm{kg}$, respectively $(P<0.001)$.

Mortality in cell dose groups 1, 2 and 3 amounted to 7, 5, 9\% $(d+30)$, and $8,11,14 \%(d+60)$, respectively. Multivariate analysis did not reveal a significant influence of quartilebased CD34+ cell dose on mortality. Even very low CD34+ cell doses $\left(<1.0 \times 10^{6} / \mathrm{kg}\right.$ and $\left.<2.0 \times 10^{6} / \mathrm{kg}\right)$ did not impact early mortality. Conditioning intensity proved to be a significant risk factor for 30 day mortality (HR 0.35, $P=0.017$ ) irrespective of CD34+ cell dose. Further influencing variables for early mortality were not identified.

Conclusion: Early posttransplant mortality seems not to be significantly altered by low viable CD34+ cell numbers of the graft. Our data indicate that a CD34+ cell dose other than $2.0 \times 10^{6} / \mathrm{kg}$ might be applicable to define the minimum threshold for allogeneic HSCT if only viable cells are considered.

Disclosure of Interest: None declared.

\section{AB11}

Pan hypopituitarism by Manganese Brain Intoxication during Long-Term Parenteral Nutrition

M. Tanaka ${ }^{1}$, A. Pereira ${ }^{1}$, M. T. Zanella ${ }^{2}$, A. Oliveira Seixas ${ }^{1}$ J. B Silva Barban ${ }^{1}$, S. Maria F Piovacari ${ }^{1}$, A. P. Noronha Barrere ${ }^{\prime}$, F. Lucio ${ }^{1}$, J. Moura Nabarrete ${ }^{1}$, N. Hamerschlak, ${ }^{1, *}$

${ }^{1}$ Hospital Israelita Albert Einstein, ${ }^{2}$ UNIFESP, São Paulo, Brazil

Introduction: Manganese $(\mathrm{Mn})$ is an essential trace element, a constituent of parenteral nutrition (PN). However, long-term PN administration can be toxic to the brain, and there are few studies about Mn intoxication. Most of them has shown that brain deposition and some neurologic symptoms, mainly parkinsonism, as a result of $\mathrm{Mn}$ administration in PN. This intoxication is diagnosed by Magnetic Resonance Imaging (MRI).

Material (or patients) and methods: Case report of an adult patient, male, 34 years old, receiving long-term parenteral nutrition with $0.4 \mathrm{mg} \mathrm{Mn/day,} \mathrm{from} \mathrm{August} 2014$ to March 2015. He had severe Crohn's disease, XIAP Mutation. Hematopoietic stem cell transplantation (HSCT) was considered as a treatment due to severity of his disease. In March 2015 , the patient had several episodes of hypoglycemia during the day. His serum level of cortisol, testosterone, thyroid 


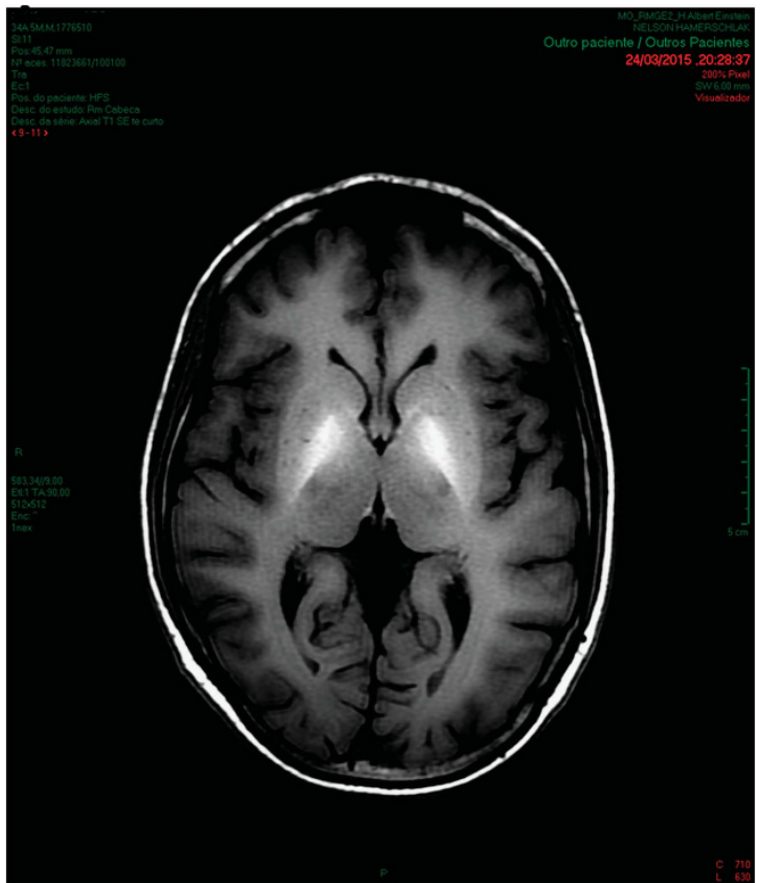

hormones were low. Brain MRI showed high spontaneous signal on $\mathrm{T} 1$ of the globus pallidus and substantia nigra more tenuous, midbrain tectum, higher brain pedundulcos, pontine integument, dentate nucleus and anterior pituitary, bilateral and symmetrical.

Results: The patient was diagnosed of Pan Hypopituitarism caused for Mn brain intoxication. The $\mathrm{Mn}$ was excluded of PN. He was undergoing HSCT in April 2015, in Bone Marrow Transplantation Division, S.Paulo, Brazil. In addition, he didn't have any HSCT complications.

Conclusion: Although $\mathrm{Mn}$ brain intoxication isn't an usual disorder in long-term parenteral nutrition, we must remember and monitor it in our patients.

Disclosure of Interest: None declared.

\section{AB12}

Use of privately banked autologous umbilical cord blood to treat brain injuries in paediatric patients

A. Smith ${ }^{1, *}$, J. Sun ${ }^{2}$, J. Allison ${ }^{2}$, M. Mimbrero-Ramirez ${ }^{1}$, K. Hussain ${ }^{1}$, J. Kurtzberg

${ }^{1}$ Smart Cells International, London, United Kingdom, ${ }^{2}$ Duke Cell and Translational Therapy Program, Durham NC, United States

Introduction: Umbilical cord blood (UCB) is an accepted hematopoietic stem cell source for patients with hematologic diseases requiring allogeneic hematopoietic cell transplantation. To date, the majority of units transplanted have been released from public cord blood banks. Recently however, there has been interest in the use of autologous UCB to treat acquired brain injuries in paediatric patients using qualified units from both public and private/family banks, particularly in the USA. The Robertson Clinical and Translational Cell Therapy Program at Duke University commenced preclinical and clinical investigations in this setting several years ago and Smart Cells International ( $\mathrm{SCl}$ ) a UK based private bank has to date supplied 6 UCB units to the Duke programme.

Material (or patients) and methods: CBUs were used to treat patients with or at risk for cerebral palsy due to hypoxic ischemic encephalopathy $(n=3)$, intraventricular hemorrhage $(n=1)$, in utero stroke $(n=1)$ and chronic encephalitis due to Herpes simplex virus and NMDA Receptor Antibodies $(n=1)$. All units were red blood cell and plasma depleted using automated Sepax ${ }^{\mathrm{TM}}$ technology prior to cryopreservation with $10 \%$ DMSO using controlled rate freezing. Total nucleated cell counts (TNCC), CD34 and viability (7-AAD) data and HLA typing results were supplied to the Duke team prior to shipment of the autologous units in monitored temperature controlled transit in vapour phase nitrogen. After premedication with acetaminophen, diphenhydramine and methylprednisolone, patients either received their total CBU as a single infusion or a portion of original product with sequential infusions according to relevant clinical trial protocol. Sequential infusions were achieved by refreezing and thawing aliquots of CBUs.

Results: At initial storage, pre-freeze mean TNCC was 555.3 $\times 10^{6}+/-329.9$ (46 to 875 ) and mean \% CD34 viability was $78.5 \%$ +/-16.4 (54 to 93.34). Following initial thawing and washing of whole CBUs by Duke, the mean recovery of TNCC was $74.5 \%$ (58 to 99.8 ), total cell viability by trypan blue was $94.8 \%$ (88 to 98 ), \% CD34 was $1.1 \%$ of CD45 cells (0.41 to 3.87), CD34 cell viability assessed with 7-AAD was $42.7 \%$ (26.39-62.95), and $5 / 6$ units grew CFUs. There were no infusion reactions or later adverse effects. Efficacy will be determined in companion placebo-controlled, randomized clinical trials.

Conclusion: This study has yielded encouraging data as the infusions were safe with no adverse effects. Provided family banks ensure that they meet standards of practice and regulatory compliance similar to public banks, the use of privately banked CBUs therapeutically, particularly in regenerative medicine is a viable prospect for the future.

Disclosure of Interest: None declared.

\section{AB13}

Comparison of two regimens of stem cell mobilization using cyclophosphamide and G-CSF in multiple myeloma M. Desole ${ }^{1, *}$, I. Hilgendorf ${ }^{1}$, C. Kunert ${ }^{1}$, L.-O. Mügge ${ }^{1}$, H. Sayer ${ }^{1}$, A. Hochhaus ${ }^{1}$, S. Scholl

${ }^{1}$ Hämatologie/ Internistische Onkologie, Universitätsklinikum Jena, Jena, Germany

Introduction: Mobilization and collection of CD34-positive stem cells for subsequent high-dose chemotherapy constitutes a standard approach in myeloma patients achieving a good remission after induction chemotherapy. There are several regimens for mobilization of stem cells including the application of different dose levels of cyclophosphamide and subsequent stimulation with G-CSF. So far, the dosage of cyclophosphamide varies between different protocols and affects both the risk of chemotherapy-associated complications and the probability of successful stem cell harvest.

Material (or patients) and methods: We retrospectively analyzed the impact of $2.5 \mathrm{~g} / \mathrm{m}^{2}$ (37 pts.) versus $4.0 \mathrm{~g} / \mathrm{m}^{2}$ (40 pts.) cyclophosphamide in 77 consecutively evaluable myeloma patients (median age 59 years, range 43-69 years) who underwent stem cell mobilization in first remission. Successful mobilization chemotherapy was defined as a stem cell harvest of at least 4 million $\mathrm{CD} 34^{+}$cells per $\mathrm{kg}$ bodyweight. In addition, important clinical factors such as infectious complications and toxicity were analyzed for both subgroups in this study.

Results: Successful stem cell mobilization was achieved in 30 out of $37(81 \%)$ and 32 out of 40 (80\%) patients, respectively. Median time to apheresis (11 vs. 12 days) and median $\mathrm{CD}_{3} 4^{+}$content of stem cell harvest (9.1 vs. 7.6 million CD34+ cells per $\mathrm{kg}$ bodyweight) did not differ significantly between both groups. Importantly, there was a significant difference of ANC nadir in favor of the cyclophosphamide regimen with $2.5 \mathrm{~g} / \mathrm{m}^{2}(0.8$ vs. $0.3 / \mathrm{nL}, P=0.01)$ while neutropenic fever was more often observed in patients who received $4.0 \mathrm{~g} / \mathrm{m}^{2}$ cyclophosphamide (16 vs. $33 \%, P=0.098$ ). Furthermore, after induction chemotherapy using bortezomib, cyclophosphamide, dexamethasone successful stem cell mobilization was achieved in 17 of 18 (94\%). One mobilization failure (4.8 million $\mathrm{CD}^{+} 4^{+/} \mathrm{kg}$ ) in this subgroup was observed in a patient who had previously received adjuvant anthracyclinecontaining chemotherapy for breast cancer. 
Conclusion: Cyclophosphamide at a dosage of $2.5 \mathrm{~g} / \mathrm{m}^{2}$ is safe and highly effective for stem cell mobilization in patients with newly diagnosed multiple myeloma in first remission after induction chemotherapy.

Disclosure of Interest: None declared.

AB14

Retrospective Analysis of the Outcome of patients receiving Grafts Immunodepleted with Alemtuzumab for Haematological Malignancies at Groote Schuur Hospital (2010-2014)

N. Novitzky, ${ }^{1, *}$, V. Thomas ${ }^{2}$

${ }^{1}$ Division of Haematology, University of Cape Town and Groote Schuur Hospital, 2Division of Haematology, Groote Schuur Hospital, Cape Town, South Africa

Introduction: Allogeneic stem cell transplantation remains an effective curative strategy in the management of haematological malignancies. Since 2006 alemtuzumab was introduced as a method for immundepletion of grafts at Groote Schuur Hospital and 137 allogeneic stem cell transplants for bone marrow failure syndromes or haematological malignancies were performed. This is a retrospective audit reviewing the outcome of transplants performed from 2010 with a minimum follow up of 12 months.

Material (or patients) and methods: Patients with haematological malignancies underwent transplantation in remission of the malignancy. The conditioning was with myeloablative dosed with busulfan $(12 \mathrm{~kg} / \mathrm{kg})$, melphalan $\left(140 \mathrm{mg} / \mathrm{m}^{2}\right)$ and cyclophosphamide $(120 \mathrm{mg} / \mathrm{kg})$. Since 2013, fludarabine $\left(150 \mathrm{mg} / \mathrm{m}^{2}\right)$ was introduced instead. TBI and cyclophosphamide was prescribed in 7 patients. Stem cells from HLA identical siblings were mobilised with filgrastim 300ug daily for 3 days and 600 ug on day 4 of the 5 day cycle. Apheresis were performed with Cobe Spectra and Optia instruments. Infection prophylaxis was with an oral quinolone, azole antibiotics and valocyclovir. GvHD prophylaxis was by immunodepletion ex vivo with alemtuzumab $1 \mathrm{mg}$ for each $10^{10}$ mononuclear cells of the graft. Patients received post-transplant cyclosporine for 90 days. Content of free alemtuzumab in the cell suspension was tested in 5 grafts by ELISA.

Results: Over the study period 54 patients were entered. The diagnoses were acute leukaemia: 32 (adverse cytogenetics in 19), advanced NHL: 13, MM: 4, MDS: 5 . Median age was 41 (range 6-60) years and 24 were female. The median CD34+ cell number was $4.5(2.6-17.8) \times 10^{6} / \mathrm{kg}$ and alemtuzumab dose "in the bag" was $5 \mathrm{mg}$ (2-10). Alemtuzumab was not detectable in the supernatant of the graft in any of the donor grafts. All patients engrafted. Twelve patients with consistently elevated ( $>3.5 \mathrm{log}$ value) CMV viral copies in the blood were treated with gancyclovir and none developed systemic disease. Acute GvHD (>grade 1) was seen in 7 patients and it contributed to the death in 2 . Twenty patients $(37 \%)$ died and at median follow up of 562 days $65 \%$ survive in remission of their malignancies. The cause of death was infection during the neutropenic period, mainly from Gram negative organisms $(n=5)$ or candidaemia $(n=2)$ and disease recurrence in 11.

Conclusion: For this younger population myeloablative conditioning followed by immunodepletion with alemtuzumab was well tolerated and associated with excellent engraftment, effective control of GvHD, low CMV reactivation and low recurrence rate of the malignancy.

Disclosure of Interest: None declared.
AB15

Clinical utility of the hematopoietic precursor cells (HPC) count compared to the $\mathrm{CD}+34$ cell count in peripheral blood for prediction of apheresis performance

C. Sossa ${ }^{1, *}$, A. Peña ${ }^{1}$, C. Perez ${ }^{2}$, M. Ochoa ${ }^{3}, H$. Melendez $^{3}$, L. Salazar ${ }^{\prime}$, S. Jimenez

${ }^{1}$ Hematologia, ${ }^{2}$ Medicina Interna, FOSCAL, ${ }^{3}$ Epidemiologia, UNAB, Floridablanca, Colombia

Introduction: The success of a bone marrow transplant depends partly on an adequate number of the infused CD34 +cells. The best time for apheresis is key. The CD34+ cell count in peripheral blood $\geq 10 \times 106 / \mathrm{I}$ is used as a criterion for performing apheresis. The hematopoietic precursor cells correlate with peripheral CD34+cells, is a fast alternative (90 seconds), no operator dependent, faster and less expensive (USD 9.8 ) vs CD $34+$ count (US \$ 110.34 ).

The objective of this work was to evaluate the clinical utility of hematopoietic precursor cells count using the $C D+34$ cell count in peripheral blood $\geq 10 \times 106 / \mathrm{I}$ as a reference.

Material (or patients) and methods: Evaluation of the diagnostic test in 109 determinations in 60 patients with bone marrow transplant indication in Clínica Carlos Ardila Lulle.

Results: The analysis showed that the cut point of $26 \mathrm{HPC} / \mathrm{ul}$ in peripheral blood has its maximum discriminatory ability to correlate with a value of CD 34+ in peripheral blood $\geq 10 \times 106$ / I, with sensitivity $78.5 \%$, specificity $85 \%$, positive predictive value $95 \%$, negative predictive value $95 \%$ and an area under the curve of 0.87 for correctly predicting the value of $C D$ $34+\geq 10 \times 106 / I$ in $79,8 \%$ of all the measurements.

Conclusion: The determination of hematopoietic precursor cells (HPC) in peripheral blood is a useful alternative given its discriminatory capacity, fast access and reduced costs.

Disclosure of Interest: None declared.

\section{AB16}

Narrative medicine in cell collection, processing and therapy

A. Van De Velde ${ }^{1, *}$, A. Devos ${ }^{1}$, G. Nijs ${ }^{2}$, C. Quisquater', F. Brugmans ${ }^{3}$, H. Daenen ${ }^{3}$, R. Suply ${ }^{4}$, E. Smits ${ }^{5}$

${ }^{1}$ Haematology, ${ }^{2}$ Center for Cell Therapy and Regenerative Medicine (CCRG), University Hospital Antwerp, Edegem, ${ }^{3}$ DigitalStories, ${ }^{4}$ Suply Hope for Cancer Immunotherapy Foundation, ${ }^{5}$ Center for Oncological Research, University of Antwerp, Antwerp, Belgium

Introduction: Narrative medicine is a medical approach that recognises the value of people's narratives in clinical practice, research and education. Narrative medicine aims not only to validate the experience of the patient, but also to encourage creativity and self-reflection in the health care provider and researcher.

Material (or patients) and methods: In a documentary short film "Cellular immunotherapy: a Digital Story of HOPE", directed by Hedwige Daenens, we use narrative medicine to describe our procedures of cell processing and cell therapy in cancer types where relapse remains a major problem and has an important impact on survival. Rupert Suply tells his medical story and guides the audience through the different steps in making a cancer vaccine.

Results: Leukapheresis, dendritic cells (DC) generation, antigen loading of DC (electroporation of Wilms' tumor 1 (WT1)-encoding mRNA), cryopreservation of WT1 mRNAelectroporated DC, timing of first vaccination, thawing of the DC vaccine, immunisation schedule, route of administration and vaccine dose are described by using documentary methods in a university hospital and research setting. Procedures in mostly restricted areas are explained in detail by qualified members of staff understandable to a general audience. 
Conclusion: Narrative medicine is a valuable tool to describe cell therapy. It promotes understanding between researcher, clinician, nurse and patient and encourages additional therapeutic options and generating new hypotheses. The documentary will be presented at this year's EBMT meeting, Nurses Group Oral session 3, Tuesday 05 April 2016.

References: 1. Smits et al. Dendritic cell-based cancer gene therapy. Hum Gene Ther 2009;20(10):1106-18.

2. Van Driessche et al. Clinical-grade manufacturing of autologous mature mRNA-electroporated dendritic cells and safety testing in acute myeloid leukemia patients in a phase dose-escalation clinical trial. Cytotherapy 2009;11(5):653-68.

3. Van Tendeloo et al. Induction of complete and molecular remissions in acute myeloid leukemia by Wilms' tumor 1 antigen-targeted dendritic cell vaccination. Proc Natl Acad Sci USA 2010;107(31):13824-9.

4. Anguille et al. Clinical use of dendritic cells for cancer therapy. Lancet Oncol 2014;15(7):e257-67.

The authors like to acknowledge Zwi N. Berneman, medical director of the Center for Cell Therapy and Regenerative Medicine, the GMP facility of the University Hospital Antwerp for haematopoietic stem cell processing and cell therapy for his pioneering work in mRNA electroporation and its application to the fields of dendritic cells and stem cells.

This project was made possible by a contribution of Biological and Medical Art in Belgium (BIOMAB), the countries leading organisation to promote interdisciplinary research between scientists and those with a passion for the synergy between Art and Science, Technology and Philosophy.

Disclosure of Interest: None declared.

\section{AB17}

Correlation of peripheral blood stem cell collection with donor characteristics and patient clinical outcome

D. Sotiropoulos ${ }^{1, *}$, K. Panteliadou ${ }^{1}$, A. Bouinta ${ }^{1}$, I. Batsis ${ }^{1}$, D. Papadopoulou', V. Papadopoulos, V. Constantinou' D. Mallouri ${ }^{1}$, I. Sakellari ${ }^{1}$, A. Anagnostopoulos ${ }^{i}$

${ }^{1}$ Department of Hematology - BMT Unit, G. Papanicolaou Hospital, ${ }^{2}$ Hematology Department, Papageorgiou General Hospital, Thessaloniki, Greece

Introduction: The number of $\mathrm{CD} 34+$ cells infused to the patient is a critical parameter for the transplantation outcome. Donor mobilization is very important for both autologous and allogeneic transplantation.

Material (or patients) and methods: In this study we have analyzed data regarding donor and patient characteristics, the apheresis procedure, the graft and the engraftment for stem cell collections that were undertaken between January 2013 and October 2015, for autologous or allogeneic transplantation. Collections were performed in Cobe Spectra apheresis apparatus. Non-parametric tests and correlation analysis were performed to identify measurements that are correlated with the concentration of CD34+ cells in donors $\left(x 10^{6} / \mathrm{Lt}\right)$, the absolute number of CD34+ cells in the graft $\left(\times 10^{6}\right)$, and the engraftment day for neutrophils and platelets.

Results: During the study period, 176 autologous apheresis procedures were performed for 147 patients diagnosed with Hodgkin lymphoma [HL: 27\%], Non-Hodgkin lymphoma [NHL: 25\%] or multiple myeloma [MM: $27 \%$ ]. The median for the concentration of CD34+ cells/lt in patients' peripheral blood was: NHL: $83, \mathrm{HL}: 128, \mathrm{MM}: 33, P<0.001$, that is patients with MM had statistically significant less CD34+ cells than patients with HL, NHL. Patient age, sex and total white blood cell count were not correlated with CD34+ cells concentration. The absolute number of CD34+ cells in the graft was correlated with donor CD34+ cells (Pearson's correlation coefficient $r=0.85, P<0.001$ ), but not with total blood volume processed or total collection volume. Transplant was actually performed in $124(85 \%)$ of the patients. Neutrophil engraftment occurred at +10 day post-transplant in patients with lymphoma and +11 in myeloma patients, $P=0.004$. Platelet engraftment occurred at +11 and +14 day in patients with lymphoma or myeloma respectively, $P=0<001$. Cell dose $\left(\mathrm{CD} 34+\times 10^{6} / \mathrm{Kg}\right)$ was significantly correlated with engraftment day for both neutrophils (Spearman's rho $=-0.56, P<0.001$ ) and platelets (Spearman's rho $=-0.38, P<0.001$ ).

For a total of 55 patients eligible for allogeneic transplantation diagnosed with AML [53\%], ALL [16\%], NHL [11\%], MM [9\%], there were 94 apheresis procedures performed in family donors (siblings [82\%], haplo-identical [9\%]). CD34+ cell mobilization was unrelated to donor age or sex; the concentration of CD34+ cells in donor's peripheral blood was correlated to white blood cell counts (Pearson's $r=0.56$, $P<0.001)$. The absolute number of CD34+ cells in the graft was correlated with CD34+ cell concentration (Pearson's correlation coefficient $r=0.8, P<0.001$ ) and the collection volume (partial correlation coefficient $=0.29$ controlling for CD34+ cell concentration, $P=0.005)$. Engraftment of neutrophils takes longer for patients with acute leukemia (13.5 vs 10 days) than in other diseases, $P<0.001$, but is not related to CD34+ or CD3+ cell count nor type of donor in multivariate analysis. Engraftment of platelets is not correlated to the disease or CD34+ or CD3+ cell count in the graft.

Conclusion: In autologous transplantations myeloma patients seem to mobilize less stem cells and that is reflected in the delayed engraftment. Interestingly, in allogeneic transplantations, there is no difference in engraftment between haploidentical and sibling donors.

Disclosure of Interest: None declared.

\section{AB18}

The evaluation of peripheral blood stem cells post-thaw viability with flow cytometry dyes propidium iodide, Annexin-V and 7-AAD reagent

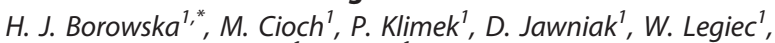
W. Knopinska-Posluszny ${ }^{1}$, M. Hus $^{7}$

${ }^{1}$ Department of Haematooncology and Bone Marrow Transplantation, Medical University Lublin Poland, Lublin, Poland

Introduction: Hematopoietic stem cell (HSC) transplantation has become a standard therapy of hematological malignancies such as Multiple Myeloma (MM), Hodgkin Lymphoma ( $\mathrm{HL})$, Non-Hodgkin's Lymphoma (NHL) and Acute Myeloid Leukemia (AML). HSC are induced to peripheral blood as a subsequence of the mobilization regimens with chemotherapy and G-CSF or plerixafor alone. The treatment permits the collection of stem cells during continuous flow apheresis. Peripheral blood stem cells (PBSC) are stored either in $-86^{\circ} \mathrm{C}$ or in liquid N2 until reinfusion. The aim of this study was to establish the viability of the $\mathrm{CD} 34^{+}$cells after cryopreservation with application of propidium iodide (PI), Annexin- $\mathrm{V}$ and 7-aminoactinomycin-D reagent (7-AAD). Moreover we evaluated if age, disease, mobilization scheme or cryopreservation period influence the hematopoietic recovery after autologous peripheral blood stem cell transplantation (APBSCT).

Material (or patients) and methods: We evaluated twenty six peripheral blood stem cell products collected from 26 patients (9 males, 17 females). The median age was 54 year (18-67). The analyzed group consists of $16 \mathrm{MM}$ patients, $5 \mathrm{HL}, 3 \mathrm{NHL}$ and 1 AML. Different mobilization regimens were applied: HDCTX, HDVEP, HDAraC or intensive chemotherapy schedules (EDAP, RDHAP, BEACOPP) with G-CSF or sometimes with plerixafor. Apheresis procedures were performed with continuous flow cell separator Com.tec (Fresenius, Germany). HSC were harvested when the pre-apheresis circulating $\mathrm{CD} 34^{+}$cell count exceeds 20 cells/ML. Apheresis products contained median $11,3 \times 10^{6} / \mathrm{kg} \mathrm{CD} 34^{+}$cells. The product was commonly divided into three EVA bags (Ethinyl Vinyl Acetate). The 10\% DMSO was applied as a cryoprotective agent. The programmed freezer was used before freezing in $-160^{\circ} \mathrm{C}$. Viability of PBSC was analyzed immediately after thawing for infusion. Viability was established according to cytometric assay protocol for propidium iodide, Annexin-V (abcam) and 


\begin{tabular}{|c|c|c|c|}
\hline PHENOTYPE & MEDIAN & MIN : MAX & DEFINITION \\
\hline $34^{+} / 7 \mathrm{AAD}^{+}$ & 1,5 & $(0,2-44,6)$ & apoptotic edls \\
\hline $34^{*} / 7 \mathrm{AAD}$ & 83,4 & (501 4-88, & Yabla cedls \\
\hline ANNEXIN ${ }^{+} / \mathrm{PI}^{+}$ & 1.7 & $(0,3-10,1)$ & necrebe calls \\
\hline ANNEXIN + PI & 3.5 & $(0,3-21)$ & early speptofic cals \\
\hline ANNEXIN'/PI & 2,1 & (85-86.6) & 4 able cells \\
\hline
\end{tabular}

7-AAD (BD stem cell enumeration kit) with BD FACSCalibur flow cytometer using BDFacsDiva software.

Results: PBSC product contained median $11,3 \times 10^{6} / \mathrm{kg} \mathrm{CD} 34^{+}$ cells $(5,3-30)$. The median time of storage was 8 weeks (5-104). With the application of flow cytometry and apoptotic dyes we determined different phenotypes: $34^{+} / 7 A^{\prime} D^{+}, 34^{+} / 7 A A D^{-}$, Annexin ${ }^{+} / \mathrm{PI}^{+}$, Annexin ${ }^{+} / \mathrm{PI}^{-}$, Annexin $/ \mathrm{PI}^{-}$. The results were expressed as a percentage of viable, early apoptotic, apoptotic cells and necrotic cells [table]. Statistical analysis shows no difference in apoptosis and necrosis in CD34+ cells harvested from patients with $M M, H L, N H L, A M L$. Also there was no correlation in cell viability and applied mobilization scheme. Slightly more viable $\mathrm{CD} 34^{+}$cells were present in PBSC cryopreserved for less than 12 weeks (Annexin ${ }^{-1} I^{-}$cells; $P=0.04)$.

Conclusion: Flow cytometry has become a method of choice of evaluation in variety of cell systems. We conclude that flow cytometric analysis of $\mathrm{PI}$, Annexin- $\mathrm{V}$ and 7-AAD binding in PBSC concentrated is a convenient markers for quality evaluation of viability status of PBSC post thaw material that undergo transplantation within APBSCT. This study shows that cryopreservation time (over 12 weeks) has a negative impact for a number of viable cells.

Disclosure of Interest: None declared.

\section{AB21}

Sterility testing of CB-derived platelet gel: validation of minimal volume of inoculum to detect the growth of both aerobic and anaerobic microrganisms

P. Bergamaschi ${ }^{1,2, *}$, B. Romano ${ }^{1}$, P. Cambieri ${ }^{3}$, D. De Vitis ${ }^{3}$, I. Sbarsi ${ }^{1}$, V. Genovese ${ }^{1}$, S. De Vitis ${ }^{1}$, A. Marchesi ${ }^{1}$, C. Perotti ${ }^{\prime}$, P. Marone

${ }^{1}$ Service of Immunohematology and Transfusion Medicine, Fondazione IRCCS Policlinico San Matteo, Pavia, ${ }^{2}$ Dept. of Biology and Biotechnology (DBB), University of Pavia, ${ }^{3}$ Microbiology Laboratory, Fondazione IRCCS Policlinico San Matteo, Pavia, Italy

Introduction: Cord blood (CB) is an alternative source for SCT in patients with haematological diseases. Criteria for $C B$ banking lead to a huge discard of units. The Italian CBB Network, following the original idea of P.Rebulla and coll., is promoting a multicenter study to produce $C B$ derivedplatelet gel (CB-PG) from units not eligible for unrelated transplantation (essentially due to low TNC count) for application in diabetic ulcers repair. In future this strategy will rescue unsuitable $C B$ to a new clinical use and reduce the discard rate favorably impacting on CBBs activity. For this reason units with TNCs $<1 \times 10^{9}$ and $\mathrm{PLT}>150 \times 10^{9} / /$ underwent a two step-procedure to prepare PLT concentrates (CB-PCs) further activated to PGs by recalcification. First, a soft spin (200gx15min) is used to obtain platelet-rich plasma (PRP), then PRP is centrifuged at $2000 \mathrm{~g} \times 15 \mathrm{~min}$ to pellet PLTs and adjust PCs concentration to $800-1200 \times 10^{9} \mathrm{PLTs} / \mathrm{l}$ for conservation at $-80^{\circ} \mathrm{C}$. As CB units may be contaminated by a variety of environmental contaminants at time of collection, sterility testing is crucial for the safety of CB-PG products. The simplest approach is to test the final product (CB-PC), volume range $5-15 \mathrm{~mL}$, despite very small specimens are available for inoculating culture vials. We previously demonstrated that PPP may represent an alternative specimen, even if a percentage of false negative results was noted especially for fungi.

Material (or patients) and methods: Here we evaluated the mixture of the two discarded fractions (PPP and pRBC) as alternative specimen for seeding BD BACTEC Plus culture media, namely Anaerobic/F for anaerobes and Peds /F for aerobes (recommended seeded volume $10-8$ and 3-1 ml, respectively). BD media were chosen because intended for testing aerobic and anaerobic bacteria and fungi and commonly available in standard laboratories. For this purpose, we inoculated fresh CBs with low bioburden $(1 \mathrm{CFU} / \mathrm{ml})$ of the following microbial isolates tested in triplicates: E. coli and S. agalactiae (aerobic bacteria), B. fragilis and $P$. acnes (anaerobic bacteria), C. albicans and $A$. niger (fungi). Then $C B s$ were processed according to CB-PG protocol. For anerobes 8,1 and $0.5 \mathrm{ml}$ of either PPP $+\mathrm{pRBC}$ or $\mathrm{PCs}$ were inoculated into Anaerobic/F vials, while for aerobes 1 and $0.5 \mathrm{ml}$ of either PPP+pRBC or PCs were inoculated into Peds/F vials; incubation time: 30 days.

Results: We evaluated the adequacy of sample, inoculum volume and $\mathrm{BD}$ media to detect the growth of aerobic/ anaerobic microrganisms in our setting. A minimal inoculum of $0.5 \mathrm{~mL}$ CPs was able to detect the growth of both aerobic and anaerobic bacteria except for Propionibacterium (slow grower) whose growth was detectable by inoculating PPP $+p R B C$ (limit volume: $1 \mathrm{~mL}$ ). For the other strains PPP+pRBC inoculations with limit volume of 1 for aerobes and $0.5 \mathrm{ml}$ for anaerobes demonstrated to be a suitable alternative sample. Conclusion: As CB-PC is a small volume product, the volume destined to quality controls is necessarily minimal. Here we demonstrated that in case of PPP $+\mathrm{pRBC}$ both sample and volume are adequate to detect major contaminants. A very tiny volume of PCs $(0.5 \mathrm{~mL})$ is also validated and is a critical step as a close system is still unavailable. Besides molecular methods able to minimize the seeding volume, our approach could be easily reproduced in contexts were such resources are not available.

Disclosure of Interest: None declared.

\section{AB22}

HLA Haploidentical Haematopoietic Stem Cell Transplantation (HSCT) for Haematological malignancies: The Greek experience in a paediatric population

A. Paisiou ${ }^{1,}$, C. M. Vadikolia ${ }^{1}$, I. Peristeri ${ }^{1}$, E. Gousetis ${ }^{1}$, E. N. loannidou ${ }^{1}$, K. Kaisari ${ }^{1}$, K. Zisaki ${ }^{1}$, G. Vessalas ${ }^{1}$, T. Maria ${ }^{\prime}$, P. Eutuhia ${ }^{1}$, V. Kitra ${ }^{1}$, S. Graphakos ${ }^{7}$

${ }^{1}$ ELPIDA Stem Cell Unit, St Sophia Hospital for children, ATHENS, Greece

Introduction: Haploidentical HSCT remains an alternative option for patients with relapsed or refractory haematological malignancies and in high risk acute leukaemias in particular, when HLA matched related or unrelated donor is not available. In literature, it has been complicated by poor engraftment, excessive regimen related toxicity and graft versus host disease (GVHD). The continuous improvement in understanding of its underlying immune mechanisms in addition to the advancements in the development of conditioning protocols, graft manipulation and GVHD prophylaxis have considerably reduced the risk of rejection and transplant related mortality (TRM).

Material (or patients) and methods: From Nov 2010 until Nov 2015, 17 patients (7 male) with median age of $12.6 \mathrm{yrs}$ (range 1,7-22 yrs)received graft from HLA haploidentical donors. Eight patients had AML (secondary from previous MDS in two out of eight), eight had ALL and one had Hodgkin Lymphoma. Disease status at the time of transplantation was Complete Remission (CR)1 in 6, CR2 in 5 and CR3/refractory disease in 6 patients. For 5 patients haploidentical was their second transplant ( $2 / 5$ due to disease relapse post allogeneic 
matched sibling donor, one with relapse post autologous SCT and 2 due to graft failure post matched unrelated transplantation). In 16/17 the donor was a parent, 1 had an haploidentical sibling. Conditioning regimen was TBI based in two, combination with Fludarabine in four, Busulfan based in 9 and Treosulfan based in the remainder 2. All but one had ATG. The origin of the graft was peripheral blood in 15 with graft manipulation (CD34+selection) and bone marrow in 2 with high-dose Cyclophosphamide post BMT. The median CD34 cells was $16 \times 10^{6} / \mathrm{kgr}$.

Results: Engraftment was achieved in all except for one, who had reinfusion of additional cells. Neutrophil and platelet engraftment occurred at a median of +15 (range 11-34d) and +30 days(range 12-62d) respectively. All but two had Donor Lymphocyte Infusions (DLI). 16 out of 17 had full donor chimerism in three months post BMT. Acute grade II-III GvHD occurred in 2 patients. Skin GvHD grade II-III appeared in 6 patients post DLI. Severe infection complications were present in 6 patients with EBV related PTLD in 4 and systemic aspergillosis in one patient. With a median follow up of 15.3 months, 9 patients are alive and well, 7 patients have died due to disease relapse and one of TRM.

Conclusion: The implementation of conditioning regimens that reduce the risk of rejection, as well as the refinement of histocompatibility testing have allowed the wider performing of haploidentical HSCT. In our limited cohort of patients, the majority achieved prompt engraftment and full donor chimerism. Severe grade II-III GvHD occurred mainly post DL and even though the incidence of moderate to severe infections was high, those were manageable in the majority of patients. Main cause of death was disease relapse or progression, as expected in this high risk group of multiply pretreated patients. Evaluation in larger cohorts, is necessary to further assess the long term benefits against the risks and to attain some insight into the immune mechanism that underlay the overcoming of HLA barrier.

Disclosure of Interest: None declared.

\section{AB23}

Clonal T cell large granular lymphocyte expansion following haploidentical haematopoietic stem cell transplantation in a patient with hodgkin lymphoma -a case report

E. Chapchap ${ }^{1, *}$, L. Kerbauy ${ }^{1}$, I. Esteves ${ }^{1}$, M. Rodrigues ${ }^{1}$, R. Helman ${ }^{1}$, F. Santos ${ }^{1}$, F. Kerbauy ${ }^{1}$, A. Ribeiro ${ }^{1}$, N. Hamerschlak ${ }^{1}$ ${ }^{1}$ Hematology and Stem Cell Transplantation, Hospital Israelita Albert Einstein, Sao Paulo, Brazil

Introduction: $\mathrm{T}$ cell large granular lymphocytes (T-LGL) expansions have been described following autologous and allogeneic haematopoietic stem cell transplantation with a low incidence, an indolent course and a distinct behavior when compared to de novo T-LGL leukaemia. There is some evidence that, even clonal expansion, may be triggered by an ethiopathogenic process involving persistent immunologic stimulation, as previously observed associations with Cytomegalovirus (CMV) infection and graft versus host disease (GVHD). To our knowledge clonal T-LGL associated with haploidentical bone marrow transplant (haplo-BMT) has not been described yet. Herein we present probably the first case of clonal T-LGL following haplo-BMT in a patient with Hodgkin lymphoma ( $\mathrm{HL})$.

Material (or patients) and methods: To describe and highlight the clinical features observed on a patient with clonal T-LGL expansion following haplo-BMT to relapsed HL. An eight colour flow cytometry technique was used to immunophenotype peripheral blood lymphocytes and the PCR for T cell receptor gama-delta (TCR- $\gamma \delta)$ gene rearrangement to detect clonality.

Results: A twenty five years old, male patient post autologous relapsed classical HL (nodular sclerosis) on a third partial remission following six cycles of brentuximab vedotin rescue has undergone an haplo-BMT and post-transplant Cyclophosphamide (Cy), non myeloablative (Flu/Cy/TBI 400 CGy), isogroup $(\mathrm{O}+)$ at July/2015. The GVHD prophylaxis consisted of tacrolimus (FK), mophetil mycophenolate and Cy. After a successful treatment of CMV reactivation at D+33 (ganciclovir) and systemic adenovirus at D+83 (cidofovir plus human immunoglobulin), this patient developed since $D+85$ until $D$ +115 (last follow-up) marked and persistent lymphocytosis (range 2.9-5.1 $\times 10^{9} / \mathrm{L}$ ) associated with mild to moderate neutropenia (range $0.6-1.4 \times 10^{9} / \mathrm{L}$ ) without any signs of GVHD or lymphoid organ involvement or autoimmunity. Patient was still using FK and donor chimerism was $100 \%$. The peripheral blood immunophenotype demonstrated an expansion of $2.7 \times 10^{9} / \mathrm{L}$ (77\% of total lymphocytes) CD3+/CD8 + T lymphocyte. Bone marrow lymphocyte immunophenotype revealed a similar pattern, corresponding to $65 \%$ of total lymphocytes. The CD4/CD8 ratio was 0.1 (peripheral and bone marrow) and Natural Killer cell percentage was $6.7 \%$ (peripheral) and $10 \%$ (bone marrow) of total lymphocytes. The circulating lymphocytes were positive to the TCR- $\gamma \delta$ gene rearrengement by PCR technique. Neither the patient nor donor demonstrated lymphocytes abnormalities before transplant.

Conclusion: Clonal T-LGL expansions can occur in patients after haplo-BMT with post-transplant $\mathrm{Cy}$ and may be associated with other viral infections, such as adenovirus. The pathogenesis, prognosis and impact on graft versus tumor effect of this condition remains poorly defined on the literature.

References: 1- Kim D, Al-Dawsari G, Chang H, Panzarella T et al. Large granular lymphocytosis and its impact on longterm clinical outcomes following allo-SCT. Bone Marrow Transplant. 2013 Aug;48(8):1104-11. doi: 10.1038/bmt.2013.5 2- Nann-Rütti S, Tzankov A, Cantoni N et al. Large granular lymphocyte expansion after allogeneic hematopoietic stem cell transplant is associated with a cytomegalovirus reactivation and shows an indolent outcome. Biol Blood Marrow Transplant. 2012 Nov;18(11):1765-70. doi: 10.1016/j. bbmt.2012.07.007.

Disclosure of Interest: None declared.

\section{AB24}

Haploidentical stem cell transplantation followed by highdose cyclophosphamide post transplant as GVHD prophylaxis in high-risk haematologic malignancies: a retrospective multicentric experience from the REP (Rete Ematologica Pugliese)

G. Pisapia ${ }^{1, *}$, D. Pastore ${ }^{2}$, A. M. Carella ${ }^{3}$, G. Palazzo ${ }^{1}$, C. Minoia ${ }^{1}$, C. Ingrosso , E. Merla ${ }^{4}$, M. Delia ${ }^{2}$, P. Carluccio ${ }^{2}$, N. Cascavilla ${ }^{4}$, G. Specchia ${ }^{2}$, P. Mazza

${ }^{1}$ Department of Hematology and Stem Cell Transplant Unit, "San Giuseppe Moscati" Hospitall, Taranto, ${ }^{2}$ Department of Emergency and Organ Transplantation (D.E.T.O.), Hematology Section, University of Bari, Bari, ${ }^{3}$ Department of Hematology and Stem Cell Transplant Unit, IRCCS "Casa Sollievo della Sofferenza" Hospital, ${ }^{4}$ Department of Hematology and Stem Cell Transplant Unit, IRCCS "Casa Sollievo della Sofferenza" Hospital, San Giovanni Rotondo, FG, Italy

Introduction: Allogeneic stem cell transplantation is the only curative option for high-risk haematologic malignancies. Haploidentical HSCT offers an opportunity of transplant in $95 \%$ of all these patients, in a simple, immediate manner. GVHD prophylaxis performed with post transplant high-dose cyclophosphamide (PT-CY), according to the Baltimore experience, has become a new standard in the Haplo-HSCT setting. To reduce the high rate of relapse reported in the Baltimore experience, in accordance with the Bacigalupo group we adopted a myeloablative conditioning regimen (MAC) in all patients.

Material (or patients) and methods: From April 2011 to February 2015, we retrospectively evaluated 43 patients (M 24, F 19), median age 37 years (range 14-69), with high-risk haematologic malignancies who underwent Haplo-HSCT followed by PT-CY, in 3 different Institutions. Diagnoses were 
$\operatorname{AML}(n=29), \operatorname{ALL}(n=9), \operatorname{CMML}(n=1), \mathrm{NHL}(n=1), \mathrm{CHL}(n=3)$; 12 patients had received a previous SCT: 10 autologous SCT, 2 URD. All patients received a MAC, 39 TBF (thiotepa, blusulfan, fludarabine), $4 \mathrm{TBI}$, fludarabine. At HSCT, 22 patients were in CR (11 in 1rst CR, 11 in 2nd or $>2$ nd CR) while 21 presented active disease. Unmanipulated bone marrow was the stem cell source for all patients. GVHD prophylaxis was performed in all patients with PT-CY $(d+3,+4$ in 35 patients; $d+3,+5$ in 8 patients), cyclosporin from $d 0$, mycophenolate from $d+1$.

Results: PMN leukocyte engraftment was reached at a median of 20 days (range 14-29). The cumulative incidence of grade II-III acute GVHD was $11.6 \%$ and moderate chronic GVHD 9\%. Cumulative TRM at $d+100$ was $23 \%$ and the relapse rate was $20.9 \%$. At $d+30,34$ patients (79\%) had full-donor chimerism. At $d+90$, a good immune reconstitution was present in $92 \%$ of evaluated patients. The actuarial 34 month DSF rate was $73 \%$ for patients in remission at HSCT and $18 \%$ for patients with active disease at HSCT. Causes of death were: relapse $(n=8)$, infections $(n=8)$, GVHD $(n=3)$, MOF $(n=2)$.

Conclusion: Haplo-HSCT with PT-CY offers a useful treatment tool in selected patients with high-risk haematologic malignancies. In this multicentric study, we confirmed that this procedure offers long-lasting remission, with limited toxicity, a low-grade aGVHD incidence and early immune reconstitution. New therapeutic strategies are needed to further reduce the relapse rate. A careful selection of alloreactive donors, HSCT performed in the early stage of disease, and an adequate adoptive immunotherapy are the future challenges.

Disclosure of Interest: None declared.

AB25

T-cell replete haploidentical donor transplantation using post - transplant Cy Preliminary report of the GATMO, Argentinian Group of Bone Marrow Transplantation

J. Martinez Rolon 1, ${ }^{*}$, J. Garcia ${ }^{2}$, J. Alberbide ${ }^{3}$, A. Basso ${ }^{4}$, J. Milone ${ }^{5}$, G. Kusminsky, G. Jarchum ${ }^{7}$, G. Remaggi ${ }^{7}$, V. Milovic ${ }^{8}$, C. Foncuberta ${ }^{9}$, E. Bullorsky ${ }^{10}$, M. Castro ${ }^{11}$, L. Feldman ${ }^{12}$, L. Riera ${ }^{13}$, G. Bentolila ${ }^{1}$, A. Basquiera ${ }^{2}$, G. Ferini ${ }^{3}$, M. Cattaneo $^{4}$, S. Yantorno ${ }^{5}$, M. M. Rivas ${ }^{6}$, S. Jarchum ${ }^{7}$, J. Real ${ }^{8}$, R. Burgos ${ }^{9}$, S. Palmer ${ }^{10}$, N. Fernandez Escobar ${ }^{12}$, P. Duarte ${ }^{13}$, G. Jaimovich ${ }^{11}$ on behalf of GATMO

${ }^{1}$ HCT Transplant, FUNDALEU, BUENOS AIRES, ${ }^{2} \mathrm{HCT}$ Transplant, Hospital privado de Cordoba, Cordoba, ${ }^{3} \mathrm{HCT}$ Transplant, Hospital Italiano de Buenos Aires, Buenos Aires, ${ }^{4}$ HCT Transplant, CETRAMOR, Santa Fe, ${ }^{5} \mathrm{HCT}$ Transplant, Hospital Italiano de la Plata, La Plata, ${ }^{6} \mathrm{HCT}$ Transplant, Hospital Austral, Buenos Aires, ${ }^{7}$ HCT Transplant, Sanatorio Alllende, Cordoba, ${ }^{8} \mathrm{HCT}$ Transplant, Hospital Aleman, ${ }^{9} \mathrm{HCT}$ Transplant, Instituto Alexander Fleming, ${ }^{10} \mathrm{HCT}$ Transplant, Hospital Britanico de Buenos Aires, ${ }^{11}$ HCT Transplant, Sanatorio Anchorena, ${ }^{12}$ HCT Transplant, Fundacion Favaloro, ${ }^{13}$ HCT Transplant, CEMIC, BUENOS AIRES, Argentina

Introduction: Hematopoietic cell transplantation (HCT) from an haploidentical related donor is an alternative option for patients (pts) without a suitable HLA-matched related or unrelated donor, or for patients at a high- risk of disease progression. In Argentina the median time from the beginning of the search of an unrelated donor to the HCT is 5.4 months. The haplo HCT platform provides an opportunity for nearly all patients to benefit from HCT.

Material (or patients) and methods: From January 2012 to June 2015, 97 pts with high- risk hematologic malignancies underwent T-cell replete haploidentical donor transplantation using post - transplant Cy (PT- Cy). Median follow- up: 6.6 months (Cl 95\% 4.5-8.8), Myeloablative (MAC)/reduce intensity conditioning regimen (RIC) $61 / 36$ pts, median age 35. 4 (5-70), female/male: 48/49. Patients underwent MAC HCT were older than RIC HCT: 30.9 vs 42.8 years-old $(P=0.001)$. $25.7 \%$ of the entire group received a previous transplant (MAC $18 \%$, RIC 39\%; $P=0.031), 37,1 \%$ were high- risk disease $(\geq 3 \mathrm{CR}$, PR,PD,Ref), MAC 29.5\%, RIC 50\%; and 62,9\% standard risk (CR1, CR2), MAC 43\%, RIC 18\% $(P=0.052)$. HCT-Cl: $0-2 / \geq 3: 87.5 \% /$
12.5\%. MAC were 39\% Bu-Flu-Cy, 20\% Flu-TBI, 16\% Bu-Flu, $10 \%$ Flu-Cy-TBI, $16 \%$ others) and RIC regimens (55\% Flu-Cy-TBI, $11 \% \mathrm{Bu}-$ Flu, $11 \% \mathrm{Bu}-\mathrm{Flu}-\mathrm{Cy}$, $5 \%$ Flu-Mel, $18 \%$ others. In MAC group $36 \%$ were $A L L, 32 \%$ AML, $6.5 \%$ CML in BC. In RIC group $42 \%$ were $\mathrm{AML}, 16.6 \% \mathrm{ALL}, 16.6 \% \mathrm{HD}, 16.6 \% \mathrm{NHL}, 5.5 \% \mathrm{MDS}$, $2.7 \%$ Multiple Myeloma. Graft source in MAC group was bone marrow (BM) 45\%, Peripheral blood (PB) 44.3\%, BM+PB 9.8\%; in RIC group: BM 27.7\% PB 72.3\%, $(P=0.089)$. GVHD prophylaxis: PT-Cy-FK-MMF MA/RIC $88.5 \% / 80.5 \%$ and $9.8 \%$ used Cs-A instead of FK 506 in both groups.

Results: Non relapse mortality (NRM) cumulative incidence (Cl) at 3 and 6 moths: $15.5 \%$ and $21.8 \%$. $66 \%$ of NRM were attributed to infections. Relapse $\mathrm{Cl}$ at 3 and 6 months: $6.5 \%$ and $14.7 \%$. NRM according to HCT-Cl: $0-2=53.6 \%$ vs $\geq 3=$ $17.2 \%(P=0.007)$. One-year overall survival (OS) was $42.9 \%$; according $\mathrm{HCT}-\mathrm{Cl}$ at 1 year: $0-2=47.1 \%$ vs $\geq 3=14.1 \%$ $(P=0.001)$. Results were not significantly different between MAC and RIC (Table). In the multivariate analysis, only $\mathrm{HCT}-\mathrm{Cl}$ was associated with survival (0-2 vs $\geq 3$ : HR $0.25 ; 95 \% \mathrm{Cl} 0.09-0.65$; $P=0.004)$.

\begin{tabular}{lccc}
\hline Variable & MAC & RIC & $p$ \\
\hline PMNcells $500 \times 10^{6} \times$ L, days after HCT & 17 & 18 & 1 \\
Platelets $25 \times 10^{9} \times \mathrm{L}$, days after HCT & 29 & 22,5 & 0.257 \\
aGVHD II-IV & $38 \%$ & $46 \%$ & 1 \\
aGVHD III-IV & $12 \%$ & $16.7 \%$ & 0.139 \\
Six-month NRM & $18.8 \%$ & $26.5 \%$ & 0.832 \\
Six-month Relapse & $12.7 \%$ & $18 \%$ & 0.219 \\
One-year OS & $46.1 \%$ & $39.1 \%$ & 0.485
\end{tabular}

Conclusion: This approach offers more and faster possibilities of finding a donor. Higher NRM was observed on RIC patients compared to the literature. This could be due to a high-risk population. We have also observed a higher incidence of a-GVHD. The influence of the HSC source used on the incidence of a-GVHD and NRM should be evaluated (PB MA/ RIC: $44.3 \% / 72 \%)$.

Disclosure of Interest: None declared.

\section{AB26}

Haploidentical Transplantation (Haplo HSCT): high survival, low incidence of graft-versus-host-disease and fast immune reconstitution using CD3 or TcRaß and CD19 depletion in the experience of Pediatric Hematology Oncology Department of Pisa

M. Menconi ${ }^{1, *}$, M. Spadea ${ }^{1}$, R. Giannelli ${ }^{2}$, S. Bernasconi ${ }^{1}$, M. Bulleri' ${ }^{2}$, G. Casazza , F. Scatena ${ }^{2}$, C. Favre

${ }^{1}$ Azienda Ospedaliero-Universitaria Pisana, U.O. Oncoematologia Pediatrica, ${ }^{2}$ Azienda Ospedaliero-Universitaria Pisana, U.O. Medicina Trasfusionale e Biologia dei Trapianti, Pisa, ${ }^{3}$ Azienda Ospedaliero Universitaria Meyer, U.O. Oncoematologia Pediatrica, Firenze, Italy

Introduction: Haploidentical transplantation is becoming a safe option for children needing an allograft. The development of new strategies in these past two decades improved GVHD control, outcomes and immune reconstitution. We implemented new strategies in graft engineering, CD3/CD19 and TCRaß/ CD19 depletion, in children with malignant and non-malignant disorders.

Material (or patients) and methods: Nineteen patients, median age $9(0,5-18)$ years, 12 male/7 female, 7 italian/12 other countries, 16 haematological malignancies (9 AML; 4 ALL; 1 MDS; 1 LNH; 1 NB), 2 Fanconi Anemia (FA), 1 Hemofagocitic Lympho Histiocytosis $(\mathrm{HLH})$, receveid haploidentical transplantation with CD3/CD19 depletion (12), TcRaß/CD19 depletion (7) between 03.05.2003 and 10.11.2015. Conditioning regimen mostly employed (in 60\% of cases) included Fludarabin, Thiotepa, Melphalan; remaining regimens included use of cyclophosphamide and Total Body Irradiation 
was used in patient with FA and MDS. ATG/OKT3 \pm Rituximab was used for GVHD prophylaxis; only 3 patients receveid cyclosporine and 2 other mycophenolate mofetil (MMF).

In all cases G-CSF-mobilized PBSC were used as graft source. CD3 or TCRa $\beta$ and CD19 depletion were performed with CliniMACS according to manufacturer's recommendations. Final graft contained a median of $7.13 \times 10^{6}$ (range $7,3 \times 10^{6}$ -

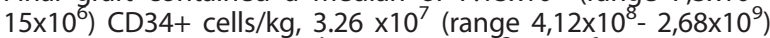
CD56+ cells $/ \mathrm{kg}, 2.08 \times 10^{4}$ (range $9.6 \times 10^{3}-1 \times 10^{6}$ ) CD3+ Tcells/ $\mathrm{kg}$ in CD3/CD19 depleted grafts; $12.3 \times 10^{6}$ (range $7.3 \times 10^{6}$. $17.2 \times 10^{6}$ ) CD34+ cells $/ \mathrm{kg} ; 3.66 \times 10^{3}$ (range $0.08 \times 10^{4}-1,23 \times 10^{5}$ ) TcRaß Tcells/kg; $1.5 \times 10^{7}$ (range $7 \times 10^{6}-33.6 \times 10^{6}$ ) TcR $\gamma \delta$ Tcells/kg in TcR aß/CD19 depleted grafts.

Several leukocyte subpopulations were analyzed in peripheral blood at days $+30 \pm 7,+90 \pm 7,+365 \pm 7$ after transplantation using multiparametric flow cytometry.

Results: Engraftment was registered in 17/19 patients, median 12 days (range 9-25) for neutrophil recovery, median 16 days (range 9-34) for platelet recovery and all patients had full donor chimerism at time of engraftment. Secondary graft failure developed in 2 patients (MDS and HLH).

GVHD was not registered in 70\% of cases. In fact, acute GVHD was registered only in 5 patients, grade 1 skin in 2 patients, and grade 1 in remaining cases.

Kaplan-Meier estimate of overall survival is $65 \%$ at median follow up of 2-years.

Immune reconstitution was fast: leukocyte subpopulations levels were normalized in about 365 days after transplantation. Mean values of $0.1 \times 10^{9}$ for CD3+ (+100 days), $0.2 \times 10^{9}$ for CD16+56+ (+30 days) and $0.1 \times 10^{9}$ for CD19+ (+100 days $)$ were early reached.

Conclusion: Graft manipulation by T-cell depletion is a highly effective method to prevent GVHD, improve outcome, and optimize immune reconstitution. Use of haploidentical transplantation can become a first line treatment for children who lack sibling donor, considering his safety and efficacy.

Disclosure of Interest: None declared.

\section{AB27}

Characterization and Assessment of CD26++ T Cells in the course of Haploidentical Stem Cell Transplantation

S. Grass ${ }^{1, *}$, J. Albrecht ${ }^{2}$, R. Klar ${ }^{1}$, A. Krackhardt ${ }^{1}$, C. Peschel ${ }^{1}$, M. Verbeek ${ }^{1}, M$. Hildebrandt ${ }^{2}$

${ }^{7}$ III. Medical Department, ${ }^{2}$ TUMCells Interdisciplinary Center for Cellular Therapies, TECHNICAL UNIVERSITY MUNICH, Munich, Germany

Introduction: CD26 is an ectoenzyme with dipeptidyl peptidase 4 (DPP4) activity expressed on a variety of cell types and is known to contribute to $\mathrm{T}$ cell activation with well-defined modulatory effects on immune response. Evidence is mounting that CD26/DPP4 expression and enzymatic activity contribute to the emergence of autoimmune disease, immune-mediated abortions and transplant rejection. We had shown previously that, in autologous stem cell transplantation (SCT), the amoun of $\mathrm{CD}_{2} 6^{++} \mathrm{T}$ cells contained in the autograft correlated with poor clinical outcome. In this study, CD26 $6^{++}$lymphocytes were investigated in patients who underwent first allogeneic haploidentical SCT.

Material (or patients) and methods: To characterize $\mathrm{CD} 26^{++}$ lymphocytes for future correlations with clinical outcome, especially chronic Graft versus Host Disease (cGvHD). Methods: Between January and October 2015, 10 eligible patients who underwent first allogeneic haploidentical SCT and their corresponding haploidentical donor were included in a prospective analysis. CD26 ${ }^{++}$lymphocytes were characterized by flow cytometry. Flow cytometric analyses were performed at defined timepoints prior to and during haploidentical transplantation. In addition, the serum concentrations and the enzymatic activity of SCD26/DPP4 were measured with commercially available enzyme-linked immunsorbent assay (ELISA) kits.
Results: $\mathrm{CD} 26^{++}$lymphocytes were identified to have a unique phenotype $\left(\mathrm{CD}^{+}, \mathrm{CD}^{+}, \mathrm{CD}_{161^{+}}, \mathrm{CD} 4 \mathrm{RO}^{+}, \mathrm{CCR} 7^{+}\right)$. The numbers of these cells showed substantial temporal heterogeneity after transplantation, and clinical data are currently collected to be matched with flow cytometric results and laboratory findings.

Conclusion: $\mathrm{CD}^{2} 6^{++}$lymphocytes define a unique set of $\mathrm{T}$ memory cells that have been postulated previously to possess chemoresistance and T-cell repopulating capacity. Given that Adenosine Deaminase (ADA) is anchored to T cells via CD26, the high density of CD26 is a critical prerequisite for chemoresistance, longevity and protection of a T cell population with obvious pathophysiological importance. Long-term follow up data are expected to allow the assessment of the clinical importance of this distinct subset in T-cell reconstitution, immune competence and chronic GvHD.

Disclosure of Interest: None declared.

\section{AB28}

Outcomes of high grade gastrointestinal gvhd post-hsct in children

A. Yesilipek ${ }^{1, *}$, V. Uygun ${ }^{1}$, H. Daloglu ${ }^{1}$, S. Özturkmen ${ }^{1}$, G. Karasu ${ }^{2}$ ${ }^{1}$ MedicalPark Antalya Hospital Pediatric BMT Unit, Bahcesehir University School of Medicine, Antalya, ${ }^{2}$ MedicalPark Göztepe Hospital Pediatric BMT Unit, Bahcesehir University School of Medicine, Istanbul, Turkey

Introduction: Our study intend to bring out the risk factors and clinical course of high grade gastrointestinal system (GIS) GVHD in children.

\begin{tabular}{|c|c|}
\hline Characteristic & Value \\
\hline \multirow[t]{6}{*}{ Diagnosis } & Acute Leukemia 7 (25\%) \\
\hline & MDS $7(25 \%)$ \\
\hline & Fanconi anemia 5 (18\%) \\
\hline & Immunodeficiency 4 (14\%) \\
\hline & Hemoglobinopathy 2 (7\%) \\
\hline & Other 3 (11\%) \\
\hline \multirow[t]{3}{*}{ Donor and HLA match } & Sibling $\mathrm{n}: 2$ \\
\hline & $\begin{array}{l}\text { Parent } \mathrm{n}: 7 \text { (four full-match, three } \\
\text { haploidentical) }\end{array}$ \\
\hline & $\begin{array}{l}\text { Unrelated n:19 (six 10/10 and } \\
\text { thirteen 9/10) }\end{array}$ \\
\hline \multirow[t]{2}{*}{ Conditioning Regimen } & Myeloablative n:23 \\
\hline & Reduced Intensity regimen n:5 \\
\hline \multirow[t]{3}{*}{ Cell Source } & Bone Marrow n:19 (68\%) \\
\hline & Peripheral Blood n:8 (29\%) \\
\hline & BM+PB n:1 (3\%) \\
\hline \multirow[t]{3}{*}{ GVHD prophylaxis } & CsA and MTX n:17 \\
\hline & CsA and MP n:5 \\
\hline & Other $\mathrm{n}: 6$ \\
\hline $\begin{array}{l}\text { Median (range) onset day } \\
\text { of GVHD }\end{array}$ & $14(5-217)$ \\
\hline $\begin{array}{l}\text { Median (range) onset day } \\
\text { of GIS GVHD }\end{array}$ & $21(5-224)$ \\
\hline First day of stage 3 & $32(7-230)$ \\
\hline \multicolumn{2}{|l|}{ Treatment modalities } \\
\hline Photopheresis & 20 \\
\hline Mesenchymal stem cells & 18 \\
\hline ATG & 6 \\
\hline MTX & 6 \\
\hline Sirolimus & 6 \\
\hline $\begin{array}{l}\text { Other (rituximab, } \\
\text { infliximab..) }\end{array}$ & 3 \\
\hline Survival & OAS \\
\hline Dead & 13 (46\%) 2,4 months $(1,4-6,4)$ \\
\hline Alive & 15 (54\%) 17,3 months $(3,0-56,3)$ \\
\hline
\end{tabular}


Material (or patients) and methods: This is a retrospective analysis of 28 pediatric patients presented with a clinical diagnosis of stage 3 and 4 acute GVHD of the GIS who were selected from allogeneic hematopoetic stem cell transplantation (HSCT) performed. The demographics, the regimen used for conditioning and GVHD prophylaxis, clinical characteristics of GVHD including follow-up, laboratory parameters during GVHD, treatment modalities used for GVHD, response assessment in every week, complications of GVHD, and survival data were recorded.

Results: Patient and transplant characteristics were summarized in Table 1.

Conclusion: Discussion Overall survival at 3 months after the onset of stage 3 or 4 gut GVHD was $54 \%$. Better outcome than the adult data might be related with more usage of bone marrow as a stem cell source or different characteristics of pediatric gastrointestinal system. In general, GVHD started 14 days post-HSCT, extended to GIS GVHD in a week and progressed to high grade GIS GVHD in 10 days. The initial day of GVHD, days to extending to GIS GVHD and progressing to high grade did not influence the mortality. Melphalan and ATG usage in conditioning regimen and the drugs used in GVHD prophylaxis were not associated with survival. Low albumin level at any time of the severe GIS GVHD was associated with high mortality possibly due to more inflammatory GIS state of lost patients. Because ATG usage in the treatment of GIS GVHD significantly increased the mortality in our study, it should not be used in a routine manner. Better but not significant outcome with non-immunosuppressants seems a better treatment approach. Today best approach to GIS GVHD is to prevent and recognize it early. Treatment without immunosuppressive therapy like Photopheresis and mesenchymal stem cells seems a better approach which deserves further research with more patients. Disclosure of Interest: None declared.

\section{AB29}

Transfusion associated graft versus host disease in a patient treated for myeloma multiplex - a case report

A. Zivanovic-Ivic ${ }^{1}$, D. Stamatovic ${ }^{1, *}$, B. Balint ${ }^{2}$, O. Tarabar $^{1}$, G. Ostojic ${ }^{2}$, O. Radic-Tasic ${ }^{3}$, M. Elez ${ }^{\prime}$, Z. Tatomirovic ${ }^{3}$, L. Tukic $^{\prime}$, S. Marjanovic ${ }^{i}$

${ }^{1}$ Military medical academy, Clinic of haematology, ${ }^{2}$ Military medical academy, Institut of transfuziology, ${ }^{3}$ Military medical academy, Institut of pathology, Belgrade, Serbia

Introduction: Transfusion-associated graft-vs-host-disease (TA-GVHD) is rare but usually fatal complication of transfused cellular blood components. The incidence of TA-GVHD is uncertain. Diagnosis of TA-GVHD may be difficult. This disease is characterized by fever, rasch, abdominal pain, diarrhoea, pancytopenia and liver disfunction 2-30 days after transfusion. Mortality rate is very high. Death typically occur about 51 days after the transfusion. We describe a fatal case of TA-GVHD that occurred after transfusion of blood in a patient who has been treated for multiple myeloma.

Material (or patients) and methods: Case report: In a 49 year old men patient $(\mathrm{Pt})$, diagnosis of Myeloma Multiplex was established in 2008. Pt received six cycles of chemotherapy (VAD) prior to autologus SCT. The maintenance therapy was applied after transplantation. One year after the transplantation relapse occured and secondary SCT had been done in pt (in decembar 2009.). Posttransplanted autologous hematopoietic reconstitution was done timely. Two weeks after transfusion in patients occur fever, nausea,vomiting and diarrhea with normal biochemistry. Infection were excluded. Twenty-three days after transfusion on physical examination noted skin rash and patechie all over the body. His respiratory, cardiovascular and central nervous system examination was normal. Laboratory data revealed pancytopenia and liver disfunction. Histology of bone marow was normal, while the histology of skin confirmed TA-GVHD.

Results: After the evidence TA-GVHD was treated with pulse steroids, then the triple immunosuppressive therapy
(Cyclosporin A, Mycophenolate mofetil, steroids). During treatment leads to the development of infection. Clinical application of treatment has led to a loss of fever, diarrhea and skin rash with recovery of renal function, of liver function and of blood count parameters. Six months after transfusion leads to deterioration of general condition and development of severe infectious syndromes (reactivation of cytomegalovirus, blood culture grew Pseudomonas aeriginosa), pancytopenia, heart and liver failure. The therapy is turned off CsA, continued implementation Mycophenolate mofetil and again implementation steroids. Eight months after transfusion leads to worsening TA-GVHD and death.

Conclusion: The diagnosis was based on the clinical condition of the patient, fever, nausea, vomiting, diarrhea, skin rash and petechiae all over the body, kidney and liver weakness, but the diagnosis of TA-GVHD was confirmed by biopsy of the skin. The patient was treated with triple immunosuppressive therapy (Cyclosporine A, Mycophenolate mofetil, steroids), with the development of severe infectious syndrome as a complication. Lethal outcome for TA-GVHD, but patient survival was four times longer than the average. Due to its rarity, TA-GVHD requires further investigation in order to define uniform approach and develop treatment and transfusion guidelines.

Disclosure of Interest: None declared.

\section{AB30}

Development of acute graft-versus-host disease post allogeneic stem cell transplantation results in significant episodes of recurrent and prolonged hospitalisations which are with associated a significant economic burden to stem cell transplant units

A. Innes ${ }^{1,2, *}$, J. Apperley ${ }^{1,2}$, Z. Allwood ${ }^{2}$, S. Altaf ${ }^{2}$, A. Chaidos ${ }^{1,2}$ E. Kanfer ${ }^{1,2}$, S. Kirschke, D. Milojkovic ${ }^{1,2}$, D. MacDonald ${ }^{1,2}$ J. Pavlu ${ }^{1,2}$, A. Rahemtulla ${ }^{1,2}$, D. Slade ${ }^{2}$, R. Szydlo ${ }^{1,2}$, E. Olavarria $^{1,2^{\prime}}$, I. Gabriel ${ }^{1,2}$

${ }^{i}$ Department of Haematology, Imperial College London, Hammersmith Hospital Campus, ${ }^{2}$ Centre for Haematology, ${ }^{3}$ Pharmacy, Imperial College NHS Trust, London, United Kingdom

Introduction: Acute graft versus host disease (aGvHD) remains a significant cause of morbidity and mortality following allogeneic haematopoietic stem-cell transplantation (allo-SCT). Treatment options for aGvHD are limited, frequently sub-optimal and often associated with considerable sideeffects. Corticosteroids (CCS) remain first-line therapy for aGvHD, however even in those who respond (SR-GvHD) $(<50 \%)$, therapy related morbidity including fungal infections or viral reactivation is high. Patients refractory to CCS (NRGvHD) have limited subsequent treatment options and there is no clear consensus for their use. Moreover, responses are often inadequate and associated with significant complications. While the novel treatment strategies for aGvHD are costly, the indirect costs of aGvHD and the complications of current treatment strategies are less well delineated.

Material (or patients) and methods: In the current economic climate with finite resources, we sought to objectively quantify the economic burden of aGvHD on our institution. We retrospectively analysed data for consecutive transplants over a 3-year period with respect to initial transplant bed stay and subsequent GvHD related re-admissions. We stratified groups by the presence or absence of $\mathrm{aGvHD}$, as well as further analysing differences between SR and NR-GvHD. The costs of these differences were calculated.

Results: Between January 2012 and December 201495 patients underwent allo-SCT within our institution from sibling $(n=43$, $45 \%)$ or unrelated $(n=52,55 \%)$ donors. The incidence of grade II-IV aGvHD was $47 \%(n=45) .25$ patients had SR-GvHD (27\%) and $20(21 \%)$ had NR-GvHD. In NR-GvHD patients the mean number of subsequent aGvHD treatments was 2 [range 1-4]. Development of aGvHD was associated with a significantly longer transplant admission, mean stay 36 days in patients without GvHD vs 46 days in those with SR-GvHD $(P=0.03)$ and 
50 days in those with NR-aGvHD $(P=0.003)$. Those with aGvHD patients had significantly more re-admissions in the first year post transplant (mean 1.1 without GvHD vs $2.3(P=0.006)$ and $2(P=0.05)$ in SR and NR-GvHD respectively) resulting in longer overall inpatient days (58 vs $91 \quad(P=0.003)$ and 90 days $(P=0.001))$. No differences were seen between SR and NRGvHD, which is likely to reflect the higher death rates seen with NR-GVHD as a competing risk for admission.

Utilising current hospital tariffs the additional hospital stay associated with aGvHD resulted in a mean excess cost of $€ 44,637$ and $€ 46,968$ for each SR-GvHD and NR-GvHD patient respectively culminating in an average annual institution cost of $€ 688,980$ from bed stay alone. Moreover, those with NR-GvHD had significantly more episodes of CMV reactivation $(0.95$ episodes) compared to no GvHD $(0.32)(P=0.01)$ or SR-GvHD (0.4) $(P=0.01)$. This is likely to further increase the economic burden of NR-GvHD and further cost analysis including antiviral, antifungal and diagnostic costs is underway.

Conclusion: The additional cost burden of aGvHD is significant for the transplant community. Strategies for steroid reduction to achieve reduced hospital inpatient stays are crucial for patient morbidity and to achieve institutional financial stability. Additionally these data highlight the need for improved strategies in NR-GvHD.

Disclosure of Interest: None declared.

\section{AB31}

The role of prolonged ECP therapy İ bronchiolitis obliterans after allo-hsct

B. Erer, ${ }^{1, *}$, F. GULEN ${ }^{2}$, S. Yilmaz ${ }^{1}$

${ }^{1}$ Pediatric BMT Center, Yeditepe University, Istanbul, ${ }^{2}$ Pediatric Pulmonology and Allergy, Ege University, Izmir, Turkey

Introduction: Chronic GVHD (cHVHD) continues to be a major complication after allo SCT. The lung involvement and development of bronchiolithis obliterans (BO) are particularly serious and life threathining conditions. Despite the advanced medical and cellular therapy the mortality and morbity are still high. Extracorporeal photopheresis treatment revealed a significant improvement of skin CGVHD, and several studies indicated steroid sparing effect on CGVHD in general. Howeover the effect of ECP on lung function in patients with BO after HSC and lung transplantation is controversial. Selective downregulation of T-cell-mediated immunity is thought to explain the effect of extracorporeal photopheresis in the treatment of GvHD.

Material (or patients) and methods: We report a case of extensive CGVHD (skin+lung) in 17 years old girl affected by B-ALL who received unmanipulated bone marrow transplant in the 2nd remission of disesase from her HLA = ABO\# brother. Full donor engrafment was documented on day +21 . She developed grade II-III acute skin CGVHD responded to steroid therapy (metihylprednisolon $2 \mathrm{mg} / \mathrm{kg}$ ). She was discharged on day +41 . Subsequently she has had several admittions to the hospital due to severe hemorrhagic cystitis. 10 months after transplant she developed extensive sclerodermiform skin CGVHD. The patient refused the steroid therapy, thus the PUVA therapy was given for 6 months. There was a significant response, except the permanence of lichenoid lesions localized on both ankles. The immunosupressive therapy (IS) has been gradualy reduced and stopped. Six months later she presented with dry cough and dispnea; wheezing and inspiratory squeaks were present on thoracic examination in absence of infection. Based on HRCT of the chest and PFT the diagnosis of BO was made. The next 6 months the symptoms, RFT and radiologic findings (HRCT) impaired despite the aggressive immunosuppressive therapy. Long course of ECP program has been added to the IS therapy. Results: She has performed 3 courses of ECP therapy. At the end of the second course of ECP therapy, cessation of disease progression was observed. She has completed 18 months of ECP therapy. Clinical symptoms have disappeared, PFT and HRCT of chest have drammaticaly improved.
Conclusion: The effect of ECP in the treatment of cGVHD may be correlated to the duration of therapy. A prolonged ECP therapy should be considered as a therapeutic option in the treatment of BO after allojeneic HSCT.

Disclosure of Interest: None declared.

\section{AB32}

Comparison of two apheresis devices in mononuclear cell collection for extracorporeal photopheresis in refractory graft versus host disease

E. González ${ }^{1}$, C. Pascual ${ }^{1, *}$, L. Solan ${ }^{1}$, S. Redondo ${ }^{1}$, A. Pérez-

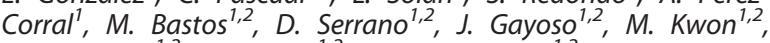
P. Balsalobre ${ }^{1,2}$, J. Anguita ${ }^{1,2}$, J. L. Díez-Martín ${ }^{1,2}$

${ }^{1}$ Hematologia, Hospital Gregorio Marañon, ${ }^{2}$ Instituto de investigación sanitaria Gregorio Marañón, Madrid, Spain

Introduction: Extracorporeal photopheresis (ECP) is an effective cell therapy in graft-versus-host disease (GVHD) after bone marrow transplantation, which requires an optimal mononuclear cells (MNC) collection through apheresis devices for further manipulation.The end of this study was to compare the semiautomatic COBE Spectra (Terumo BCT ${ }^{\circ}$ ) and the new automated Spectra OPTIA (Terumo BCT ${ }^{\circ}$ ) in terms of efficacy and safety in ECP.

Material (or patients) and methods: All patients with refractory acute and chronic GVHD in treatment with ECP were retrospective studied, from June 2014 to May 2015. Patients were alternatively assigned with either apheresis device. Peripheral blood sample was removed to the patients before and after each leukocyte apheresis (LA) procedure and also had a sample of each product just before reinfused. We perform the cell blood count (CBC) in Unicell DHX800 (Beckman Coulter) in all samples. Collection efficiency (CE) of white blood cell (WBC) and total mononuclear cells (TMNC) and platelet loss $(\mathrm{PL})$ were calculated as:

WBC CE\% $=$ [product WBC- $\mathrm{NC} \times 10^{6} / \mathrm{mL} \times$ product volume $\mathrm{mL} /$ preapheresis $\mathrm{NC}\left(\times 10^{6} / \mathrm{mL}\right) \times($ Vol processed $\mathrm{Ml}-\mathrm{AC}$ volume $\mathrm{MI})$ ] $\mathrm{x} 100$

TMNC CE\% $=$ [product $\mathrm{MNC} \times 10^{6} / \mathrm{mL} \times$ product volume $\mathrm{mL} /$ preapheresis MNC $\left(\times 10^{6} / \mathrm{mL}\right) \times$ (vol processed $\mathrm{mL}$-ACvolume $\mathrm{mL})] \times 100$

$\mathrm{PL}=[$ Preapheresis count-Postapheresis count/ Preapheresis count] $\times 100$

* In all cases it was complete with saline solution to a total volume of $300 \mathrm{ml}$. It were compared patient's CBC before and after the $L A$, the $C B C$ of the product and the procedure variables with both devices, using non-parametric methods (U Mann-Whitney).

Results: A total of 200 procedures (100 performed in COBE and 100 in OPTIA) were included, carried out in 11 patients ( 8 with chronic GVHD and 3 with acute GVHD). Central venous access was required in 10/11(90.9\%) patients. CBC results before and after LA were similar. In OPTIA group, the processed volume, the volume of the product, the percentage of loss of platelets, the amount of ACD used and the amount of ACD in the product, were significantly lower. The hemoglobin and the hematocrit were higher in the OPTIA group, and TMNC count was similar with both devices. Notably, TMNC CE was greatest in the OPTIA group.There were no differences in the duration of the procedure. Details are shown in Table 1. The procedures were well tolerated with both devices, and only mild episodes of hypocalcemia were recorded in four procedures (two in OPTIA group, and two in COPE group).

Conclusion: In our study, the Spectra OPTIA proved very effective and save in collecting MNC for ECP in GVHD.Because of this, we could consider shortening the time of collection with this device. The lower PL is very important because these patients often have low platelet counts. It also provides the advantage of being automatic and lower use of ACD what may reduce the hypocalcemia risk. The hemoglobin and the hematocrit were higher in the OPTIA group, but in the established limits for proper irradiation. 


\begin{tabular}{|c|c|c|c|}
\hline Varisble & COBE $(n=100)$ & OPTIA $(n=100)$ & p value \\
\hline WBC count $\left(\times 10^{5} / \mathrm{ml}\right)$ & $11,5(4,6-20,7)$ & $15,9(7,9-26,3)$ & 0,002 \\
\hline TNC $\times 10^{8}$ & $34,50(13,87 \cdot 62,10)$ & $47,70(23,92-79,12)$ & 0,002 \\
\hline Lymphocytes $\left(\times 10^{6} / \mathrm{ml}\right)$ & $7,3(2,1-12,6)$ & $8,0(2,8-15)$ & 0,325 \\
\hline Lymphocytes \% & $67,6(45,8-79,6)$ & $47,5(31,2-70,3)$ & $<0,001$ \\
\hline Monocytes $\left(\times 10^{6} / \mathrm{ml}\right)$ & $2,7(0,8-6,05)$ & $4,4(0,6-9,7)$ & 0,051 \\
\hline Monocytes \% & $25,2(14,4-43)$ & $27,9(7,3-51,6)$ & 0,890 \\
\hline TMNCx10 & $33(11,25-61,42)$ & $42(15-77,85)$ & 0,095 \\
\hline TMNCx $10^{3} / \mathrm{Kg}$ & $57.5(18.9-98.3)$ & $74.8(25.6-120)$ & 0,116 \\
\hline $\mathrm{Hb}(\mathrm{g} / \mathrm{dl})$ & $0,1(0,1-0,2)$ & $0,2(0,1-0,3)$ & $<0,001$ \\
\hline Hto (\%) & $0,3(0,2-0,5)$ & $1,0(0,5-1,6)$ & $<0,001$ \\
\hline PLT count $\left(x 10^{6} / \mathrm{ml}\right)$ & $379(106-831)$ & $141(68,5-263,5)$ & $<0,001$ \\
\hline WBC Collection Efficiency (\%) & $7,6(4,1-14,1)$ & $17,8(9,9-30,2)$ & $<0,001$ \\
\hline TMNC Collection Efficiency (\%) & $21(11,3-35,5)$ & $39(26,1-55)$ & $<0,001$ \\
\hline Loss of platelets (\%) & $33,6(26,5-41,1)$ & $22,9(18,3-28,1)$ & $<0,001$ \\
\hline Blood volumen (ml) & $4038(3905-4831)$ & $3952(3727-4858)$ & 0,441 \\
\hline Volume processed (ml) & $7702(6628-8667)$ & $6063(5525-6925)$ & $<0,001$ \\
\hline Blood volume processed (fold) & $1,7(1,6-1,9)$ & $1,5(1,4-1,5)$ & $<0,001$ \\
\hline Product volume(ml) & $104(94-115)$ & $80(60-100)$ & $<0,001$ \\
\hline Procedure time(min) & $148(140-162,7)$ & $147(133-163,7)$ & 0,480 \\
\hline ACD used $(\mathrm{ml})$ & $649(564-742)$ & $601(514-690)$ & 0,009 \\
\hline ACD in the product (ml) & $12(11-14)$ & $9(7-11)$ & $<0,001$ \\
\hline
\end{tabular}

Data presented as median and RIQ. HB: hemoglobine. HTO: hematocrit. CE: colletion efficiency. TNC: total nuclear cells. TMNC: total mononuclear cells.

References: Mononuclear cell collection for extracorporeal photochemotherapy: a study comparing an automatic and a semiautomatic apheresis device. Del Fante et al. Transfusion. 2013; 53:2027-2033.

Disclosure of Interest: None declared.

\section{AB33}

Transplant-Associated Thrombotic Microangiopathy (TA-TMA) in a patient with acute GVHD after allogeneic hematopoietic stem cell transplantation successfully treated with eculizumab

I. Donnini ${ }^{1, *}$, M. Di Gioia ${ }^{1}$, C. Nozzoli ${ }^{1}$, A. Gozzini ${ }^{1}$, S. Guidi ${ }^{1}$, R. Saccardi ${ }^{\prime}$, A. Bosi ${ }^{1}$

${ }^{7} B M T$ UNIT, AOU CAREGGI, Florence, Italy

Introduction: Transplant-associated thrombotic microangiopathy (TA-TMA) represents a challenging and potential lifethreatening complication after alloHSCT. This syndrome occurs approximately in $10 \%$ to $20 \%$ of patients after alloHSCTs, ${ }^{1}$ and it is characterized by inflammation, thrombotic microangiopathy, hemolytic anemia, thrombocytopenia, and evidence of organ damage, particularly renal and neurological involvement. The mortality rate is approximately $30-80 \%$. The treatment is problematic and no generally effective therapies are available. Therapeutic options consist in a wide range of treatment such as Plasma exchange (PEX) or immunosuppressive drugs, however lacking in high response rate due to the unclear TA-TMA pathophysiology. Recent data suggest that endothelial injury and complement activation could be involved in the pathogenesis of TA-TMA. Eculizumab, a monoclonal antibody against terminal complement, may be an innovative and effective therapeutic option for TA-TMA.

Material (or patients) and methods: We report a case of TATMA in a recipient of alloHSCT for acute lymphoblastic leukemia $\mathrm{B} \mathrm{Ph}$ negative. 34-year old male who underwent alloHSCT from unrelated donor 9/10 HLA compatible with total nucleated cells $10.54 \times 10^{8} / \mathrm{Kg}$ and $\mathrm{CD} 34+8.44 \times 10^{6} / \mathrm{Kg}$.
Conditioning regimen consisted of TBI 1200 cGy and Cyclophosphamide $60 \mathrm{mg} / \mathrm{Kg}$ on days -3, -2. Anti Thymocyte Globulin, Methotrexate and Ciclosporine (CsA) were given for Graft versus Host Disease (GvHD) prophylaxis.

Results: At day +23 of reinfusion the patient experienced cutaneous acute GvHD (skin 2, global 1) treated with prednisolone $2 \mathrm{mg} / \mathrm{Kg}$. At day +30 , due to worsening of cutaneous rash and liver dysfunction (elevated transaminase and bilirubin levels), the patient started treatment with Etanercept $25 \mathrm{mg} / \mathrm{sc}$ for a total of 3 infusions. Meanwhile the $\mathrm{LDH}$ level was increased up to $1024 \mathrm{U} / \mathrm{L}$ and was associated to anemia, trombocytopenia and renal deterioration; schistocytes were detected at the peripheral blood smear (10 HPF) and urinanalysis revealed a protein concentration of $8.052 \mathrm{mg} /$ $24 \mathrm{~h}$. CSA was replaced with mycophenolate mofetil and the patient started treatment with plasma and PEX daily, stopped after 4 cycles due to trombocytopenia. Few days later patient developed peripheral paresthesia and hypostenia; cranial MRI was performed, with no pathological features. Despite recovering renal status and hb level, C3 and C4 levels were inferior to normal range and patient started treatment with Eculizumab 900 mgs for a total of 2 infusions. After the first dose of Eculizumab there was a marked improvement of performance status, LDH, hb level and platelet count (Fig. 1); schistocytes in the peripheral blood decreased until disappearance. At +113 diarrhea gr3 appeared and a colonoscopy showed ulcerative mucositis and inflammatory infiltrates. Patient received Infliximab $600 \mathrm{mg} / \mathrm{mq}$ (4 doses). Actually patient is at +200 and experience only mild cGvHD (NIH scale: mouth 1 , eyes 1 ).

Conclusion: This report support the potential effective activity of Eculizumab in the treatment of life-threatening TA-TMA even when it is associated with refractory acute GvHD.

References: 1. Chapin J, Shore T, Forsberg P et al. Hematopoietic transplant-associated thrombotic microangiopathy: case report and review of diagnosis and treatments. Clin Adv Hematol Oncol. 2014 Sep;12(9):565-73.

Disclosure of Interest: None declared. 
TA-TMA: Clinical course \& Response to Treatment

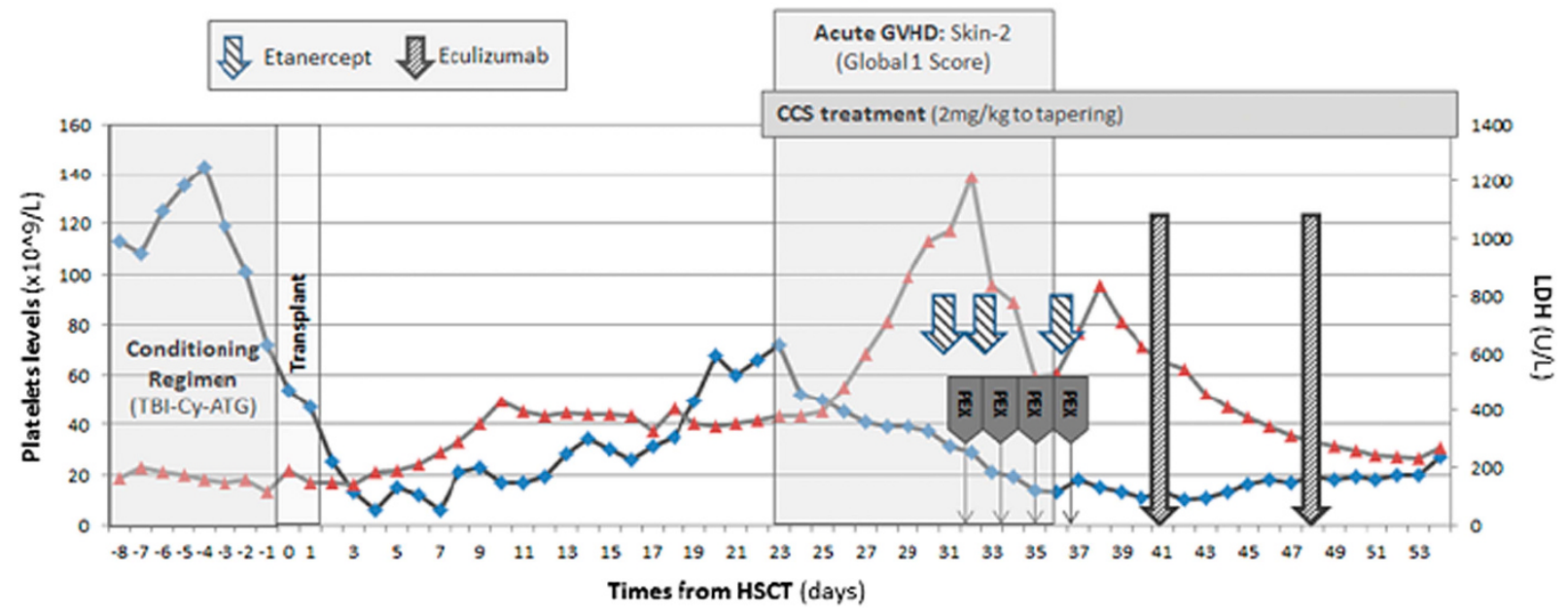

AB34

Use of skin direct therapy with phototherapy in association with extracorporeal photopheresis in graft versus host disease: experience of National Cancer Institute/INCA/Brazil

M. M. Silva ${ }^{1, *}$, M. C. moreira ${ }^{1}$, L. arcuri ${ }^{1}$, R. D. C. tavares $^{1}$ S. lermontov ${ }^{\prime}$, F. oliveira ${ }^{1}$, I. Mota ${ }^{1}$, D. lerner $^{1}$, R. Araujo ${ }^{\prime}$, L. F. Bouzas ${ }^{1}$

${ }^{1}$ bone marrow transplant unit, National Institute of Cancer, rio de janeiro, Brazil

Introduction: We have been using at our institution phototherapy - PUVA and UVBNB as skin direct therapy associated to ECP to treat different manifestations of cutaneous GVHD for the last 24 years. ECP is indicated to patients not responsive to steroids, those with IS toxicity or those who need early withdrawal of IS.

Material (or patients) and methods: All patients treated with phototherapy since 1990 were prospectively evaluated in a single center institution. PUVA and UVBNB was performed with standard machine and UVA initial dose was based on skin type. ECP was performed with UVAR photophresis system and liquid psoralen. PUVA and UVB-NB could also be combined with ECP for different clinical cutaneous manifestations or as ECP maintenance therapy. Complete response (CR) was defined as more than $75 \%$ of clinical response and partial response (PR) between $50 \%$ and $75 \%$. Patients who had less than 30 phototherapy sessions were considered early for evaluation (EA).

Results: Puvatherapy was used in 67 patients to treat lichen planus like and sclerodermic chronic GVHD with no visceral involvement. UVBNB was used in 25 patients for vitiligo, limited lichen planus like chronic GVHD, follicular keratosis, patients with psoralen oral intolerance and children. ECP was reserved to treat 43 patients with extensive sclerodermic fasciitis CGVHD, systemic CGVHD and 13 patients with acute GVHD corticosteroid-refractory. Patients treated with ECP had more severe cGVHD $(P<0,01)$. ECP was combined with PUVA in six patients and in UVBNB in 13.

Conclusion: PUVA and UVBNB are topical direct therapies and should be considered in patients in whom long-term systemic IS is antecipated. Phototherapy as topical therapy on the skin associated with ECP helps in the treatment of sclerotic CGVHD extensive and vitiligo that are often seen in the same patient. This study shows encouraging results and suggests that phototherapy treatment results in more rapid reduction of immunosuppression.
References: 1. Greinix HT, Worel N, Just U, et al. Extracorporeal photopheresis in acute and chronic graft-versus-host disease. Transfus Apher Sci. 2014 Jun;50(3):349-57. 2. Radojcic V, Pletneva MA, Couriel DR. The role of extracorporeal photopheresis in chronic graft-versus-host disease. Transfus Apher Sci. 2015 Apr;52(2):157-61. 3. Ballester-Sánchez,M.Á. NavarroMira, B. de Unamuno-Bustos,et al. The Role of Phototherapy in Cutaneous Chronic Graft-vs-Host Disease: A Retrospective Study and Review of the Literature Actas Dermosifiliogr. 2015;106(8):651-657. 4. Garbutcheon-Singh KB,FernándezPeñas P. Phototherapy for the treatment of cutaneous graft versus host disease. Australas J Dermatol. 2015 May;56(2):93-9. 1. Abu-Dalle I, Reljic $T$, Nishihori $T$, et al. Extracorporeal photopheresis in steroid-refractory acute or chronic graftversus-host disease: results of a systematic review of prospective studies. Biol Blood Marrow Transplant. 2014 Nov;20(11):1677-86. 2. Greinix HT, Worel N, Just U, et al. Extracorporeal photopheresis in acute and chronic graft-versus-host disease. Transfus Apher Sci. 2014 Jun;50(3):349-57.

Disclosure of Interest: None declared.

\section{AB35}

Immunotherapy in the treatment of severe refractory chronic graft versus host disease

M. Elez ${ }^{1, *}$, O. Tarabar ${ }^{1}$, S. Mirosavljevic ${ }^{1}$, B. Balint ${ }^{2}$, O. Tasic Radic ${ }^{3}$, V. Skuletic ${ }^{3}$, S. Marjanovic ${ }^{1}$, M. Malesevic ${ }^{1}$, L. Tukic ${ }^{1}$, D. Stamatovic ${ }^{1}$

${ }^{1}$ Clinic of hematology, ${ }^{2}$ Institute for transfuziology, ${ }^{3}$ Institute for pathology, Military Medical Academy, Belgrade, Serbia

Introduction: Chronic graft versus host disease (cGvHD) is major predictor of long term survival after allogeneic stem cell transplantation (HSCT). Similarities with autoimmune diseases are due to $B$ lymphocyte (B-Ly) dysregulation and thus abnormal production of various auto antibodies (Ab). Using of chimeric monoclonal anti CD20 antibody (Rituximab) is based on their "in vivo depletion" of B-Ly and thus we estimate efficacy and safety of Rituximab in the therapy of refractory severe cGvHD.

Material (or patients) and methods: Seven patients, M/F 5/2, have received Rituximab after failure to standard treatment (Methylprednisolone, Cyclosporine A, Mycophenolate mofetil, Azathioprim, PUVA...). Median age was 29 years (18-49). All of them have received HLA identical sibling transplant with stem cells from peripheral blood in 5 and bone marrow in 2 cases. Despite standard prophylaxis, 4 of them had acute GvHD 
grade 1-2 and all of them had severe cGvHD with lichenoid, sclerodermic or pemphigoid skin feature, buccal mucosa involvement with sicca syndrome. Extracutaneous manifestation included lung in 4, liver in 4 and thrombocytopenia in 3 cases. One patient had CMV infection prior to exacerbation of cGvHD. We applied Rituximab in usually recommended regimen ( $375 \mathrm{mg} / \mathrm{m}^{2}$ weekly for 4 weeks).

Results: Exact responses were measured with Schirmer test, spirometry, liver function parameters and hematological findings. Good effect was observed in 5 patients $(71,4 \%)$ even after first application. Best results were achieved concerning mucosa (significant reducing of sicca syndrome) and skin (improvement of turgor, lichenoid changes and pemphigoid reconstitution). Lung and liver function were improved as well, but it should be mentioned that other immunosuppressant were also given (particularly Mycophenolate mofetil and Azathioprim). Three patients had severe infectious complication, two of them have died from sepsis, and one of them have developed abscesses pulmonalis in fourth week of treatment. Conclusion: Results from our modest data suggests that monoclonal anti CD20 antibody in the treatment of severe refractory cGvHD is encouraging but needs further investigation.

Disclosure of Interest: None declared.

AB36

Human herpesviruses in oral chronic Graft-versus-Host Disease

M. Mravak-Stipetic ${ }^{1}$, L. Desnica ${ }^{2}$, D. Pulanic ${ }^{3, *}$, R. ServentiSeiwerth ${ }^{2}$, R. Vrhovac ${ }^{2}$, I. Sabol ${ }^{4}$, E. Prenc ${ }^{2}$, M. Grce ${ }^{4}$, D. Nemet ${ }^{2}$, S. Z. Pavletic ${ }^{5}$

${ }^{1}$ Oral Medicine, School of Dental medicine University of Zagreb, ${ }^{2}$ Division of Hematology, Department of Internal Medicine, University Hospital Centre Zagreb, Zagreb, ${ }^{3}$ School of Medicine, University of Osijek JJStrosmayer, Osijek, ${ }^{4}$ Division of Molecular Medicine, Rudjer Boskovic Institute, Zagreb, Croatia, ${ }^{5}$ National Cancer Institute, National Institutes of Health, Bethesda, United States

Introduction: Human herpesviruses (HHVs) are commonly found in general population but also in patients after allogeneic hematopoietic stem cell transplantation (HSCT) where they may cause severe complications. Particularly, cytomegalovirus (CMV) infection was significantly more frequent among adults and was associated with a higher risk of developing acute graft-versus-host disease (aGvHD) (1-3). Therefore, the aim of our study was to determine prevalence of particular HHVs in patients with oral chronic graft-versus-host disease (cGvHD) and possible role in these patients.

Material (or patients) and methods: This pilot study enrolled 36 cGvHD patients who are part of a larger multidisciplinary cGvHD project at the University Hospital Center Zagreb, Croatia. Patients were evaluated for clinical and cGvHD characteristics; severity of cGvHD and oral involvement were determined by using established $\mathrm{NIH}$ Consensus criteria. Cytobrush swabs from oral mucosa were collected from all patients. Specimens were analyzed by specific polymerase chain reaction (PCR) of several HHVs (CMV, Epstein-Barr virus EBV, herpes simplex virus 1 and 2, and varicella zoster virus VZV). Data were evaluated descriptively and Chi-square test was used to calculate statistical significance.

Results: 36 cGVHD patient were enrolled, of which 18 men (average age 37.8 ) and 18 women (average age 40.9).Twenty patients had diagnostic signs of oral cGvHD (55.6\%), 13 were $(36.1 \%)$ without, and $3(8.3 \%)$ had distinctive signs of oral cGvHD. Overall, in 18 of 36 patients (50\%) no HHVs were identified, $3 / 36(8.33 \%)$ had CMV, $13 / 36(36.11 \%)$ had EBV, 2/33 (6.06\%) VZV and no HSV 1 and 2 were found, respectively. EBV was statistically significantly found more often than the second most common CMV $(P=0.0046)$. There was no statistically significant difference in HHVs between patients with and those without oral cGvHD.
Conclusion: Although proportion of patients with CGvHD, especially patients with oral CGvHD was EBV positive, indicating the possibility that EBV is more common in chronic GVHD, further studies with control groups are necessary to determine the role of EBV in CGVHD. Our preliminary data also indicate that the use of molecular methods to determine the presence of HHVs is valuable method in the follow-up of CGvHD patient particularly by using oral cytobrush specimens for PCR analysis as less invasive alternative method for monitoring these patients.

References: de Oliveira PG1, Ueda MY, Real JM, de Sá Moreira E, de Oliveira JS, Gonçalves MV, Ginani VC, de Oliveira Felix OM, Seber A, Novis Y, Rocha V, Granato CF, Arrais-Rodrigues C. Simultaneous Quantification of the 8 Human Herpesviruses in Allogeneic Hematopoietic Stem Cell Transplantation. Transplantation. 2015 Nov 9. [Epub ahead of print].

Marr KA1. Delayed opportunistic infections in hematopoietic stem cell transplantation patients: a surmountable challenge. Hematology Am Soc Hematol Educ Program. 2012;2012:26570. doi: 10.1182/asheducation-2012.1.265.

Sellar RS1, Peggs KS. Recent progress in managing graftversus-host disease and viral infections following allogeneic stem cell transplantation. Future Oncol. 2012 Dec;8(12): 1549-65. doi: 10.2217/fon.12.153.

Disclosure of Interest: None declared.

\section{AB37}

bleedHD: a Tool for Assessment of Bleeding and Graftversus-Host Disease in Hemato-oncological Patients

M. Egger ${ }^{1}$, J. Halter, ${ }^{2}$, L. Infanti ${ }^{2,3}$, A. Plattner ${ }^{4}$, S. Schefer ${ }^{5}$, L. Angerer, J. Kistler', J. Passweg ${ }^{2}$, A. Buser ${ }^{1,2}$, A. Holbro ${ }^{1,2}$

${ }^{1}$ Blood Transfusion Service, Swiss Red Cross, ${ }^{2}$ Hematology, University Hospital Basel, ${ }^{3}$ Blood Transfusion Center, ${ }^{4}$ Swiss Red Cross, Basel, ${ }^{5}$ Getunik AG, ${ }^{6}$ Kistler Web Activities, Zürich, Switzerland

Introduction: Bleeding complications and Graft-versus-Host Disease (GVHD) are severe complications in hematooncological patients and patients undergoing allogeneic transplantation (HSCT). A systematic and prospective assessment of these complications is essential in order to evaluate therapeutic interventions, optimize patient care and thus improve patient outcome. Different bleeding scores to be used in clinical practice but also for research purposes have been developed, including the WHO and BSMS scores. ${ }^{1,2}$ Recently, the National Institute of Health (NIH) updated their recommendations for diagnosis and staging of GVHD. ${ }^{3}$ For the prospective and systematic evaluation of bleeding complications and GVHD, we developed an electronic tool with the aim to improve patient care and for clinical research purposes.

Material (or patients) and methods: In collaboration with IT-specialists we developed a browser based electronic tool for assessment of bleeding and GVHD according to the published guidelines. This tool (bleedHD) works on different devices and thus allows efficient bedside assessment. Collected data include patient's and disease characteristics, type of intervention, type and site of bleeding as well as GVHD features, according to the published guidelines.

Results: bleedHD is a secured browser based, user-friendly tool and was improved over time and validated on a large number of patients. The current available tool allows easy collection of patient characteristics including names, date of birth, gender, age, diagnosis and interventions (type of transplantation and stem cell source). Thereafter, the assessor can choose between assessment of bleeding (either WHO or BSMS or both) or GVHD or both. According to the published NIH guidelines, GVHD can be scored in case of first diagnosis, by every single organ (organ scoring), by activity assessment and finally by self-reporting by the patient himself. The user will be guided step-by-step through the different scoring systems. Assessments can be interrupted at any time and resumed later. bleedHD automatically gives an overview over time and scores 
of each patient. During assessment, explanations, additional information and pictures can be retrieved allowing correct assessment also for not experienced users.

All the data are safely stored on an appropriate server. Complete data can be exported as CVS file for further analysis and research purposes.

In our experience bleedHD significantly improves the accuracy of bleeding and GVHD assessment in an easy, fast and straightforward approach. Scoring patients became much more convenient and easy. Additionally it allows a prospective bedside assessment and clear demonstration of clinical changes in case of therapeutic interventions. Physicians and nurses from other institutions, which are involved in patient care can have access to bleedHD and also assess GVHD during follow-up.

Conclusion: bleedHD is an innovative and easy tool for an accurate, efficient and prospective assessment of bleeding complications and GVHD in hemato-oncological patients. This can improve patient care and outcome. Additionally, it can be a useful tool for clinical research in the therapy of GVHD and to further investigate the efficacy of platelet transfusions or other interventions in thrombocytopenic patients.

References: 1. Middelburg RA et al. Vox Sang. 2013.

2. Webert KE et al. Transfusion. 2012.

3. Jagasia MH et al. Biol Blood Marrow Transplant. 2015.

Disclosure of Interest: None declared.

\section{AB38}

The impact of extracorporeal photopheresis in acute and chronic graft-versus-host disease with multiorganic involvement

S. Cortez ${ }^{1, *}$, D. Hernandez ${ }^{1}$, M. Canales ${ }^{1}$, A. Sastre ${ }^{1}$, V. Jimenez ${ }^{1}$, A. Viejo

${ }^{1}$ Hematology, Hospital Universitario La Paz, Madrid, Spain

Introduction: Graft versus host disease (GVHD) is a lifethreatening complication of allogenic hematopoietic stem cell transplantation, which has a wide range of pleomorphic manifestations at multiple organs. Extracorporeal photopheresis (ECP) is an immunomodulatory therapy that provides encouraging results in skin GVHD but also in multiorganic involvement (skin, gut and liver). The ECP reverses GVHD through the reduction in response of donor effector $T$ cells and production of donor Tregs.

Material (or patients) and methods: We retrospectively analyzed 24 patients with refractory acute and chronic GVHD treated in our center with ECP from September 2009 to October 2015. The median age was 16 (range 2-61), the main primary disease was acute myeloid leukemia. There were 12 patients with aGVHD that were classified according to the degree of severity in grade II and III 67\% and grade IV 33\%, 12 patients with CGHVD patients were classified according the global scoring of $\mathrm{NIH}$ consensus into moderate $75 \%$ and severe $25 \%$. Skin was the organ mostly involved (79\%), while $37 \%$ of patients had multiorgan involvement. ECP was performed using the online system THERAKOS, and the off line system using the Spectra Optia separator, and the UVA device MACOPHARMA. Patients with aGVHD received 2-3 consecutive days of ECP at weekly intervals until response and then tapered to every 2 to 4 weeks, patients with cGVHD received 2 consecutive sessions at 2 week intervals.

Results: The Overall response rate (ORR) for aGVHD and cGVHD was $75 \%$ and $91 \%$ respectively. The ORR for patients with aGVHD grade II-III was $100 \%$ and grade IV was $25 \%$. In moderate and severe CGVHD the ORR was 66 and $100 \%$ respectively. We analyzed organ specific complete response (CR) rates in patients with aGVHD and CGVHD (graphic 1), in skin involvement $\mathrm{CR}$ was achieved in $78 \%$ and $72 \%$ (aGVHD and cGVHD), in multiorganic involvement (skin, liver and gut) the CR was $50 \%$ and $67 \%$ respectively. Seven patients died, the main cause of death was infectious disease. The procedure was well tolerated, only $\mathrm{NCl}$ grade I adverse events were observed.

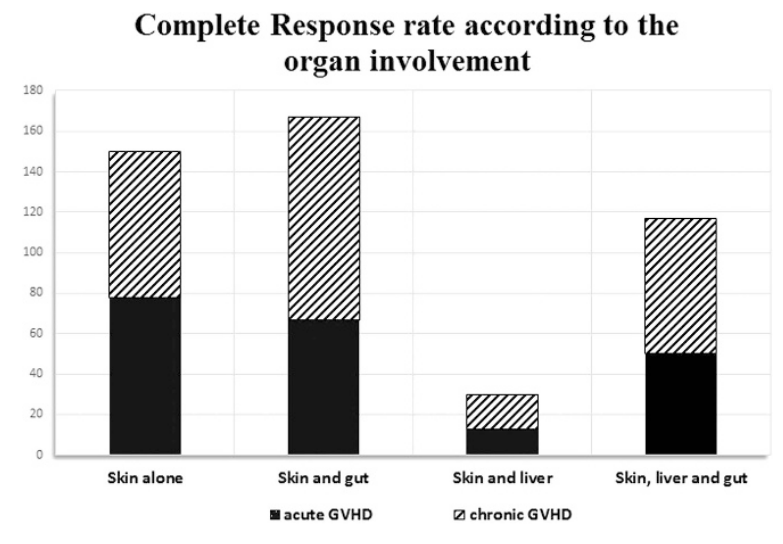

Conclusion: In our experience the use of ECP for aGVHD, had favorable rates of response especially at early stages. There is tendency for higher responses in aGVHD with cutaneous involvement. We observed significant rates of responses in moderate and severe CGVHD. We found that ECP has a considerable activity against multiorganic cGVHD. The early intervention with ECP improves the poor outcome of patients with steroid refractory GVHD.

References:

1.Greinix $\mathrm{H}$, Besien $\mathrm{K}$, et al. Progressive improvement in cutaneous and extracutaneous chronic graft-versus-host disease after a 24-week course of extracorporeal photopheresis results of a crossover randomized study. American society for blood and marrow transplantation 2011;17(12):1775-82

2. Hart J, Shiue L, et al. Extracorporeal photopheresis in the treatment of graft-versus-host disease: evidence and opinion. Ther Adv Hematol 2013;45:320-334.

3. Berger M, Albiani R, et al. Extracorporeal photopheresis for graft-versus-host disease: the role of patient, transplant, and classification criteria and hematologic values on outcome-results from a large single-center study. Transfusion 2015;55:736-747.

Disclosure of Interest: None declared.

\section{AB39}

Treatment adherence to vaccination schedule after hematopoietic stem cell transplantation

A. J. Palma Vallellano ${ }^{1, *}$, I. Vázquez-Pastor ${ }^{7}$, S. Ramírez García ${ }^{7}$ E. Gil Espárraga', G. García-Donas ${ }^{1}$, K. Gómez Correcha ${ }^{1}$ J. N. Rodríguez Rodríguez ${ }^{1}$, Y. Skiredj Akkor ${ }^{7}$, R. Zapata Bautista ${ }^{1}$ M. A. Ruiz Cobo ${ }^{1}$, I. Rodriguez Morales ${ }^{1}$

${ }^{7}$ Hematología, Hospital Juan Ramón Jiménez, Huelva, Spain

Introduction: After autologous hematopoietic stem cell transplantation (HSCT) it exists a period of time with an increased risk of infectious diseases because of immune dysfunction. For this reason, it is advisable to administer several vaccines beginning 6-12 months after transplantation. In our hospital, the haematologist responsible for posttransplant monitoring establishes the prescription of vaccination, both the dates and the number of doses, including vaccines against Stp. Pneumoniae, $H$. inluenzae type $b$, C. tetani, C. diphtheria, Poliovirus and Influenzavirus. After this, patients are sent to the Primary Care Trust (PCT) where the general practitioner is responsible for the correct completion of the set prescription, indicating the administration to the nursing unit.

Material (or patients) and methods: The aims of the current study are: a) to assess treatment adherence to the vaccination schedule elaborated in our unit and determine reasons for lacking of adherence, if this is the case; b) to identify administrative problems (lack of vaccines, absence of collaboration between the general practitioner and the haematologist responsible, etc.); 
c) to describe adverse reactions related to vaccines (fever, rash, etc.).

Methods: Descriptive and retrospective study of patients who underwent autologous stem cell transplantation in our hospital from May 2008 to October 2013, so it has been possible to complete the vacunal schedule. Information was obtained by telephone interviews and reviewing medical histories. Patients were questioned about: a) the administration of every prescribed vaccine; $b$ ) reasons why vaccines were not administered, if this occurred; c) administrative problems in obtaining them; d)administration of additional vaccines not prescribed by the haematologist; $d$ ) local and systemic adverse reactions after vaccine administration.

Results: 58 transplanted patients (34 male, 24 female) with a median age of 51.5 years, diagnosed of Multiple Myeloma 24 (41.4\%), Hodgkin Lymphoma 10 (17.2\%), NHL-B 18 (31\%), NHL-T 3 (5.2\%), Acute Leukaemia 2 (3.4\%), Plasmatic Cell Leukemia $1(1.7 \%)$. Of these, 51 (88\%) patients answered the questionnaire and 7 (12\%) were unable to be contacted due to exitus or change in hospital. Of those who completed the questionnaire, $42(82.3 \%)$ recieved all the prescribed vaccines by the haematologist, 7 patients reported having problems with availability. From 9 (17.6\%) patients who have not been administered any vaccines, 6 of them were for haematologist indication (due to progression of disease), and 3 obey to patient refusal. From all vaccinated patients, only 2 (4.8\%) were given an additional vaccine, not included in the original calendar (Influenza A). From those vaccinated, 7 (16.7\%) reported any of the following adverse reactions: fever (4), asthenia (1), flu-like symptoms (1), and local skin reaction (1). Conclusion: In our hospital unit, even though there were a relatively small number of patients, the degree of adherence observed was quite high and without any relevant incidence. We consider that collaboration with the Primary Care Trust in the administration of the post-transplant vaccination schedule has an important role when treating and caring for our patients.

Disclosure of Interest: None declared.

AB40

Screening of hepatitis B virus before hematopoietic cell transplantation

A. Nakaya ${ }^{1, *}$, S. Fujita ${ }^{1}$, A. Satake ${ }^{1}$, T. Nakanishi ${ }^{1}$, Y. Azuma ${ }^{1}$, Y. Tsubokura ${ }^{1}$, M. Hotta ${ }^{1}$, H. Yoshimura ${ }^{1}$, K. Ishii ${ }^{1}$, T. Ito ${ }^{\prime}$, S. Nomura?

${ }^{1}$ First Department of Internal Medicine, Division of Hematology and Oncology, Kansai Medical University, Osaka, Japan

Introduction: It has been reported that reactivation of hepatitis B virus (HBV) could be fatal in patients with malignancy following systemic immunosuppressive chemotherapy, especially combined with rituximab, or hematopoietic cell transplantation (HCT). Many guidelines regarding HBV reactivation following chemotherapy recommends that $\mathrm{HB}$ surface antigen ( $\mathrm{HBsAg}$ ), antibody to $\mathrm{HB}$ core antibody (anti-HBc), and antibody to HB surface antibody (anti-HBs) should be tested before the chemotherapy is initiated. Japanese guideline for HBV infection in whom received chemotherapy was published in 2009; however it remains a few cases reported who developed fulminant hepatitis from HBV reactivation following chemotherapy. This fact implies that the guideline is not properly observed. Therefore we conducted this study to evaluate whether HBV screening tests before HCT were employed according to the guideline in the HBV-endemic region of Japan.

Material (or patients) and methods: We retrospectively analyzed 127 patients who were treated with allo- or auto-HCT between 2006 and 2014 at Kansai Medical University Hospital. Results: A total of 127 patients were studied. 43 patients underwent allo-HCT and 84 patients underwent auto-HCT. All patients were examined on $\mathrm{HBsAg}$ before the HCT. 2 patients (1.5\%) were proved to be $\mathrm{HBsAg}$ positive carrier, who received nucleoside analog against HBV thereafter. Only 5 patients (3.9\%) were investigated neither on $\mathrm{HBCAb}$ or $\mathrm{HBsAb}$. Although 25 patients $(19.6 \%)$ were seropositive either for $\mathrm{HBcAb}$ or $\mathrm{HBsAb}$, 19 patients (76\%) among them were not examined on HBV-DNA as follow-up. Fortunately no fulminant hepatitis was experienced, 2 patients developed late-onset reactivation. These 2 were patients who underwent auto-HCT. One was a HBV carrier who had received prophylactic nucleoside analog during the whole course of therapy, whose HBV-DNA level resulted in undetected. She developed reactivation of HBV with long interval after autoHCT. The other had occult infection of HBV, who converted to late-onset reactivation after auto-HCT. Neither of them developed hepatitis after they were retreated with nucleoside analog against HBV.

Conclusion: Improvement was achieved by the guideline on management against HBV reactivation following chemotherapy. While it did not pervade perfectly even in the HBVendemic region of Japan. Most hematologists would recognize how important to examine the HBV serostatus before the HCT; however they are afraid to lose the following blood test regarding HBV. The guideline might be complicated to observe the follow-up study from the point of view. Thus, the guideline is preferable to be simplified and the institutional surveillance should be established to encourage the follow-up study. If other medical staff composed of nurses, pharmacologists, and laboratory technicians as well as hematologists, is aware of HBV serostatus of the patient during the entire course of chemotherapy, it could be attributable to prevent from missing the follow-up study.

References: 1. JSH Guidelines for the Management of Hepatitis B Virus Infection Drafting Committee for Hepatitis Management Guidelines and the Japan Society of Hepatologyt, * Article first published online: 7 JAN 2014 DOI: 10.1111/ hepr.12269.

2. Kusumoto S, Tanaka Y, Suzuki R, et al. Monitoring of Hepatitis B Virus (HBV) DNA and Risk of HBV Reactivation in B-Cell Lymphoma: A Prospective Observational Study. Clin Infect Dis. 2015 May 1. pii: civ344.

Disclosure of Interest: None declared.

AB41

A fatal case of late citomegalovirus meningoencephalitis after cord blood transplantation

L. Sisinni $i^{1, *}$, M. Torrent ${ }^{1}$, L. Turón ${ }^{1}$, R. Gimeno ${ }^{2}$, I. Badell ${ }^{1}$

${ }^{1}$ Paediatric BMT Unit, Hospital Santa Creu i Sant Pau, ${ }^{2}$ Inmunology, Hospital del Mar Medical Research Institute, Barcelona, Spain

Introduction: Citomegalovirus meningoencephalitis is a rare and fatal complication after allogeneic hematopoietic transplantation. We describe a paediatric patient who developed the infection one year after cord blood transplantation although preemptive treatment and good control of blood viremia.

Material (or patients) and methods: A 15-year-old male patient with $\mathrm{Ph}+$ acute lymphoblastic leukemia in third complete remission received an unrelated 5/6 HLA matched single umbilical cord blood transplant. The patient and the donor (maternal IgG serostatus) were CMV-seropositive. The conditioning regimen was based on fludarabine, thiotepa, busulfan and anti-thymocyte globulin. Cyclosporine and a short course (14 days) of prednisone were given as GVHD prophylaxis. Because of acute grade II GVHD (gut) patient received two courses of prednisone $2 \mathrm{mg} / \mathrm{Kg} /$ day and oral budesonide starting on day +7 and +105 , respectively. Cyclosporine discontinuation started on day +205 . Intravenous Ig was administered monthly. The patient showed good engraftment and total donor chimerism although with a poor immune reconstitution (persistent low CD4 count) implying frequent CMV asymptomatic viremia. Complete molecular remission was maintained.

Results: On day +300 the patient started with apathy, memory lost, language deficit and lethargy. Brain magnetic resonance imaging showed signs compatible with meningitis. 
Cytomegalovirus was detected by quantitative PCR technique in the cerebrospinal fluid (CSF) $(23.000 \mathrm{UI} / \mathrm{ml})$ whereas it was negative in blood. An autoimmune cause was ruled out. At that moment the patient was receiving oral valganciclovir as preemptive treatment for a previous CMV viremia (maximum $3.400 \mathrm{UI} / \mathrm{ml}$ ). He was admitted to the paediatric BMT Unit to start double intravenous antiviral treatment based on ganciclovir and foscarnet. Cyclosporine was discontinued in one week. A progressive neurological worsening was seen during the next two months although negativity of CMV in CSF was achieved. The patient died on day +375 .

Conclusion: Citomegalovirus meningoencephalitis is a severe complication that can occur late after hematopoietic transplantation. Our patient experienced the fatal infection although effectiveness of preemptive antiviral treatment to clear blood viremia.

Disclosure of Interest: None declared.

\section{AB42}

Is active invasive fungal infection a contraindication for allogeneic stem cell transplantation in a patient with severe aplastic anemia?

S. Solmaz ${ }^{1, *}$, A. Korur', Ç. Gereklioğlü ${ }^{2}$, S. N. Ulusan ${ }^{3}$, C. Boğ ${ }^{1}$, H. Özdoğu

${ }^{7}$ Hematology, ${ }^{2}$ Family Medicine, ${ }^{3}$ Radiology, Başkent University Adana Adult Bone Marrow Transplantation Center, Adana, Turkey, Adana, Turkey

Introduction: Fatal infection by bacteria and invasive fungal (especially Aspergillus) is the most frequent cause of severe aplastic anemia (SAA) mortality. However, there are very few spesific reports about infections and their therapy in patients with AA. We aimed to discuss the therapy scenarios for two SAA patients who have invasive fungal infection.

Material (or patients) and methods: Case 1. A 26 year-old male patient with SAA was admitted with fever, periorbital swelling, periorbital pain, and bloody nasal discharge. The patient had been diagnosed with SAA. Computed tomography of the paranasal sinuses demonstrated a space-occupying lesion in the right maxillary and ethmoid sinus region. Histopathologic examination revealed numerous PAS-stained Aspergillus hyphae without tissue invasion. Tissue culture revealed Aspergillus fumigatus. Voriconazole administration was started.

Case 2. A 24-year-old male patient was admitted to our clinic due to pancytopenia. The patient was diagnosed with SAA and allogeneic HSCT was planned. However he had fever on day 3 of admission. Invasive pulmonary aspergillosis was suggested based on the presence of pneumonic consolidations on CT of the thorax and galactomannan positivity (1.18 index). Liposomal amphotericin B was started in therapeutical dose.

Results: Invasive fungal infections (IFI), particularly those caused by Aspergillus species, have long been recognised as a major cause of death in SAA. Mortality rate is still high in SAA patients with IFI despite antifungal therapy and even granulocyte infusion plus G-CSF administration.

A condition in which the response to immunosupressive therapy can take up to 6 months to develop leading to a neutropenic period that is typically longer than that seen after HSCT. HSCT with active IFI places the patient at a higher risk for transplant related mortality. However, HSCT without delay is only curative treatment approach in some patients.

We performed HSCT under antifungal therapy in both patients. Both patients overcame the neutropenic period and they were discharged without severe morbidity.

Conclusion: We conclude that an immediate HSCT may be life-saving and G-CSF plus granulocyte transfusion may be used as a bridge to HSCT in patients who do not satisfactorily respond to antifungal therapy although there are scarce data about the HSCT in SAA patients with active invasive fungal infection. eferences: 1. Dufour et al. Outcome of aplastic anemia in adolescence: a survey of the Severe Aplastic Anemia Working Party of the European Group for Blood and Marrow Transplantation. Haematologica 2014;99:1574-81.

2. Scheinberg P. Aplastic anemia: therapeutic updates in immunosuppression and transplantation. Hematology Am Soc Hematol Educ Program 2012;2012:292-300.

3. Wang et al. Granulocyte transfusion combined with granulocyte colony stimulating factor in severe infection patients with severe aplastic anemia: a single center experience from China. PLoS One 2014;9:e88148.

4. Quillen et al. Granulocyte transfusions in severe aplastic anemia: an eleven-year experience. Haematologica 2009;94: $1661-8$

5. Weng et al. Successful treatment of disseminated mixed invasive fungal infection after hematopoietic stem cell transplantation for severe aplastic anemia. Pediatr Transplant 2012;16:E35-8

Disclosure of Interest: None declared.

\section{AB43}

Primary antifungal prophylaxis with posaconazole vs fluconazole in early neutropenic phase after allogeneic stem cell transplantation

A. Mele ${ }^{1}$, G. Greco ${ }^{1}$, C. De Risi ${ }^{1}$, S. Sibilla ${ }^{1}$, B. Rossini ${ }^{1}$, D. Carlino ${ }^{1}$, S. Citiso ${ }^{1}$, E. Prete ${ }^{1}$, M. R. Morciano ${ }^{1}$, A. Peluso ${ }^{1}$, V. Pavone ${ }^{1, *}$ ${ }^{1} \mathrm{OU}$ Hematology and TMO, Hospital Card. G. Panico, Tricase, Italy

Introduction: Invasive fungal infections increase morbidity and mortality in the early neutropenic phase after allogeneic haemopoietic stem cells transplantation (HSCT). Posaconazole is a new oral azole with a wide spectrum activity and a safety profile.

Material (or patients) and methods: We analyze efficacy and safety of posaconazole during the early phase post allogeneic HSCT (74 recipients (70\%) from February 2008 ) in comparison with fluconazole primary antifungal prophylaxis (32 patients (30\%) up to January 2008).

Results: Groups were comparable in terms of age, underling disease, number previous chemotherapies, status at transplant, median time to HSCT, previous autotransplant, use of unrelated donor, type of conditioning, incidence of GvHD and GvHD prophylaxis. The median CD34+ infused cells was $4,8 \times 10 \wedge 6 / \mathrm{Kg}$

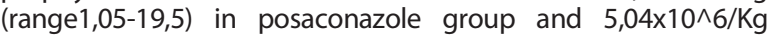
(range1,02-20,1) in fluconazole group $(P=0,54)$. Neutrophil and platelets engraftment was similar in the two groups, table 1. Incidence of febrile neutropenia episodes $(49(66 \%)$ in posaconazole vs22(68\%) in fluconazole; $P=0,79)$ and invasive fungal infections (3(4\%)in posaconazole vs $1(3 \%)$ in fluconazole; $P=0,81$ ) were comparable for both antifungal agents. However, patients receiving posaconazole had a lower incidence of empiric/preemptive antifungal therapy $(10(20 \%)$ vs $11(50 \%) ; P<0,01)$ which associated with a higher probability of systemic antifungal treatment free time $(21 \%$ vs $57 \%$ at 100 day; $P<0,01)$. The median days of antifungal treatment were 12(3-30) in posaconazole and 10(1-29) in fluconazole group $(P=0,19)$. No difference was observed in terms of 1yTRM ( $28 \%$ in posaconazole and $36 \%$ in fluconazole; $P<0,99)$ and $1 y O S(58 \%$ in posaconazole vs $55 \%$ in fluconazole; $P<0,77$ ). No adverse serious events occurred during the antifungal prophylaxis. Fifteen patients (20\%) treated with posaconazole temporarly withdrawn the drug because of gastrointestinal symptoms.

Conclusion: Our experience suggests that antifungal prophylaxis with posaconazole is well tolerated and may lead to a lower rate of empiric/pre-emptive antifungal therapy and a higher probability of systemic antifungal treatment free time period for patients in the early neutropenic phase post allogeneic HSCT.

Disclosure of Interest: None declared. 
Table1. Engraftment and results

\begin{tabular}{lcc}
\hline & Posaconazole & Fluconazole \\
\cline { 2 - 3 } & $N=74$ & $N=32$ \\
\cline { 2 - 3 } & & $5,04(1,02-20,1)$ \\
\hline CD34 infused x106/Kg,median (range) & $4,8(1,05-19,5)$ & $21(70 \%)$ \\
$>4 \times 106 K g$ & $44(64 \%)$ & $14(10-24)$ \\
Median days to PMN > 500uL (range) & $13,5(10-27)$ & $15(56 \%)$ \\
$>12$ days & $39(61 \%)$ & $16(11-56)$ \\
Median days to Platelets $>$ 20000/uL (range) & $15(10-33)$ & $13(54 \%)$ \\
$>15$ days & $30(48 \%)$ & $22(68 \%)$ \\
Febrile neutropenia, no. (\%) & $49(66 \%)$ & $11(50 \%)$ \\
FUO & $28(57 \%)$ & $9(41 \%)$ \\
Microbiological & $14(29 \%)$ & $4(18 \%)$ \\
Pneumonitis & $9(18 \%)$ & $1(3 \%)$ \\
Invasive Fungal Infections, no. (\%) & $3(4 \%)$ & 1 \\
GM+CT & 2 & $11(50 \%)$ \\
Candida Albicans Sepsys & 1 & 9 \\
Use of systemic antifungal therapy, no. (\%) & $10(20 \%)$ & 1 \\
Liposomal amphotericin B & 6 & 1 \\
Voriconazole & 1 & $28,5(13-59)$ \\
Caspofungin & 3 & $16(50 \%)$ \\
Median days af AFT, range & $12(3-30)$ & 1 \\
$>10$ days & $6(54 \%)$ & 0,01 \\
Median time spent in Hospital, range & $28,5(11-67)$ & \\
$>$ 28days & $37(50 \%)$ & \\
\hline
\end{tabular}

[AB43]

Figure 1. a) Time to sistemic antifungal treatment; b) TRM and antifungal prophylaxis; c) $O S$ and antifungal proph ylaxis
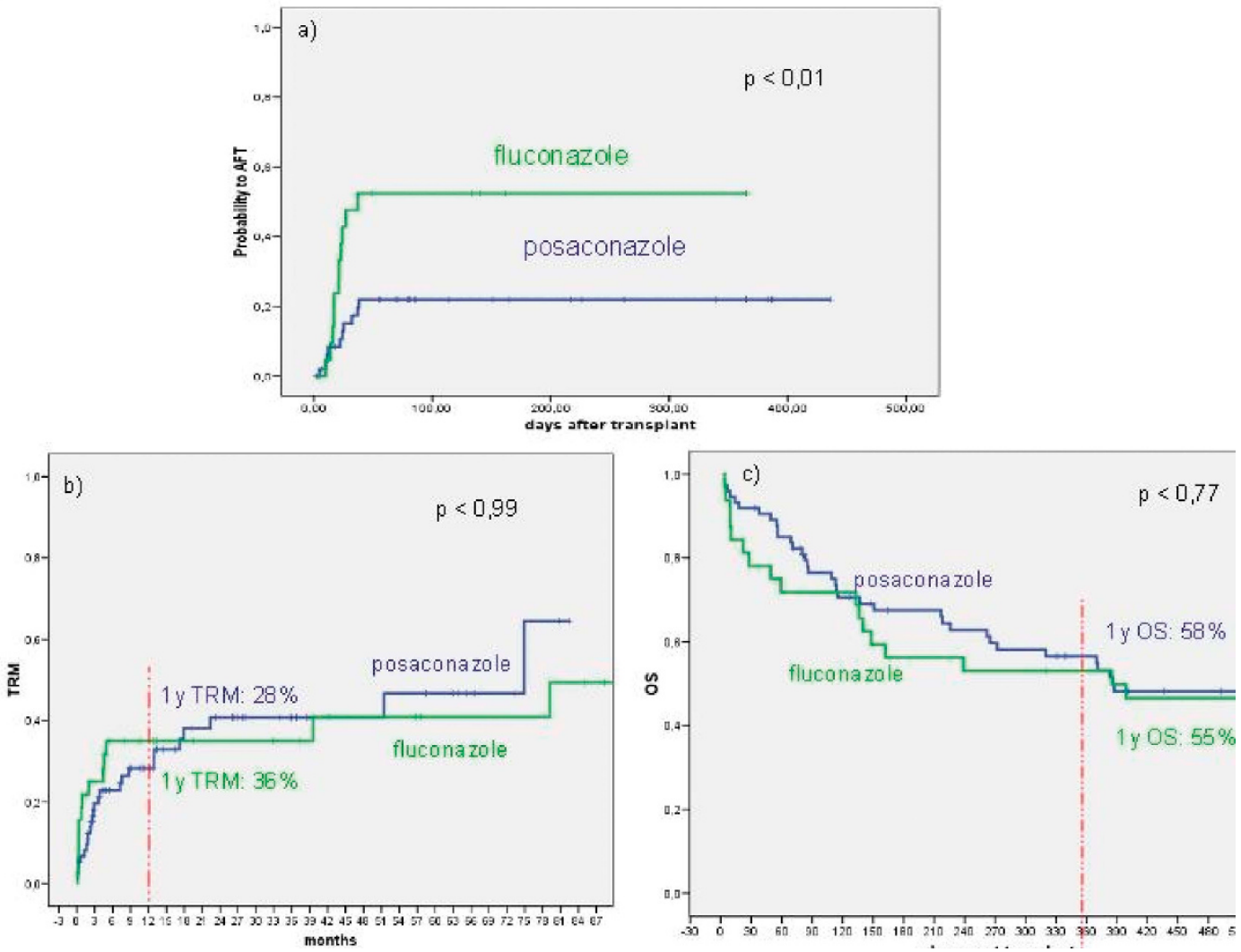
AB44

Multidrug resistant bacterial isolates - 15 years single center experience

Z. Stojanoski $i^{1, *}$, A. Pivkova ${ }^{1}$, S. Genadieva-Stavrik ${ }^{1,1}$, L. Cadievski $^{1}$, L. Cevreska ${ }^{1}$, B. Georgievski ${ }^{1}$

'Bone marrow department, University clinic of hematology, Skopje, Macedonia, The Former Yugoslav Republic Of

Introduction: Background: Infective complications are the most important factor of mortality and further morbidity in stem cells recipients. There are significant differences in local microflora in different transplant centers. Today multidrug resistant strains of bacteria are increasingly frequent in this group of patients. Extended-spectrum beta-lactamase producing Escherichia coli, Vancomicyn resistant enterococci, Methicillinresistant staphylococcus aureus and Penicillin-resistant pneumococci are major problem to manage. Aim: to evaluate the frequency of multidrug resistant bacteria in our center.

Material (or patients) and methods: Material and method: during a 15 years period we have transplanted 360 patients with different hematological malignancies. All patients were in sterile room conditioned with HEPA filters and low bacterial diet. As antibacterial prophylaxis we use Ciprofloxacine 1,0gr/ day. Empirical antibiotics regimen consisted Third-generation cephalosporin+amynoglicoside, while second line Imipenem +Vancomycin.

Results: Results: In every patient 3 times per week we monitor blood culture, CVC culture, urine culture, sputum. From all 247 bacterial isolates the distribution of MDR bacteria were: ESBL positive Escherichia coli 2 (0,8\%) VR Enterococcus 1 $(0,4 \%)$ MDR Pseudomonas aer. 1(0,4\%) Klebsiella aerogenes 2 $(0,8 \%)$ MRSA $15(6 \%)$ Acinetobacter baumanii $2(0,8 \%)$ Stenotroph. Maltophilia 2(0,8\%). The fatal outcome due to sepsis we have in 3 patients (VRE 1, MDR Pseudomonas 1and Stenotrophomonas maltophilia 1).

Conclusion: the monitoring of local microflora is mandatory for every transplant center. Empirical first-line therapy must be individualized and according to ECIL 4 escalation and deescalation strategy is necessery for better outcome in this group of immunocompromised patients.

References: Multidrug Resistant Gram Negative Bacteria in a Cohort of Hematopoietic Stem Cell Transplant Recipients.

Disclosure of Interest: None declared.

\section{AB45}

United Kingdom Oral Mucositis in Cancer Group Guidance: Second Edition

B. G. Quinn 1,*, M. Thompson ${ }^{2}$, J. Horne ${ }^{3}$, S. Hoy ${ }^{4}$, L. Fulman ${ }^{5}$, C. Frances ${ }^{6}$, S. Dargan 7 , D. Houghton ${ }^{8}$, J. Treleaven ${ }^{9}$

${ }^{1}$ Cancer, Chelsea \& Westminster Hospital, London, ${ }^{2}$ Radiography, Beatson Cancer Centre, Glasgow, ${ }^{3}$ Haematology, NHS Trust, Aberdeen, ${ }^{4}$ Cancer, Royal Marsden NHS Trust, London, ${ }^{5}$ Radiography, NHS Trust, Nottingham, ${ }^{6}$ Cancer, Beatson Cancer Centre, Glasgow, ${ }^{7}$ Palliative Care, Ashford \& St Peters NHS Trust, Surrey, ${ }^{8}$ Cancer Pharmacy, Ramsay Health Hospital, ${ }^{9}$ Haematology, Royal Marsden NHS Trust, London, United Kingdom

Introduction: Changes to the oral cavity in the transplant setting can be caused by numerous factors including the disease, the direct and indirect impact of treatments, coexisting co-morbidities and underlying oral health problems.

Material (or patients) and methods: The United Kingdom Oral Mucositis in Cancer Care (UKOMiC), a multi-professional expert group was founded in 2011 to address the challenges of oral complications secondary to disease and treatment in the transplant, cancer and supportive care setting. The first edition of the oral care clinical guidance produced in 2012 has been widely used within transplant centres in the United Kingdom and many other countries to help support and improve practice. The group has continued to disseminate the guidance, through the delivery of several national study days, numerous educational workshops and lectures, while continuing to collaborate with international organisations.
Results: This presentation focuses on the recently updated oral care guidance which is based on the most recent evidence, clinician feedback and expert opinion.The guidance continues to focus on the key principles of; an accurate assessment of the oral cavity, identification of risk factors, regular care, earlier intervention to prevent/reduce oral damage and the correct treatment interventions.

Conclusion: It is anticipated that this updated guidance will further assist health care professionals in planning and implementing oral care into everyday practice, thus reducing a significant health burden for the patient and reduce demands on limited health care resources.

Disclosure of Interest: None declared.

\section{AB46}

Successful pregnancy and delivery after myeloablative cord blood allogeneic transplantation

C. de Miguel ${ }^{1, *}$, G. Bautista ${ }^{1}$, C. Regidor ${ }^{1}$, I. Krsnik ${ }^{1}$, J. R. Cabrera ${ }^{1}$ ${ }^{1}$ Hematology, Hospital Universitario Puerta de Hierro Majadahonda, Majadahonda, Spain

Introduction: Infertility is a frequent long-term side effect among young hematopoietic stem cell transplantation (SCT) receptors ${ }^{1}$. Concern about fertility negatively affects the quality of life of the patients which frequently ask about the optimal time to address these issues and the ways to do it.

A few cases of successful pregnancies after autologous or allogeneic transplants, have been recently reported but, to our knowledge, none after cord blood transplantation (CBT).

We report the case of a young woman who successfully carried out a pregnancy with a donated oocyte, 7 years after a TBI-including myeloablative CBT.

Material (or patients) and methods: Our patient, a 27-year old woman, was diagnosed of pro-B acute lymphoblastic leukaemia in August 2006. She was treated with a national high-risk ALL protocol (PETHEMA HR-ALL 2003), with persistence of disease after induction. First complete remission was achieved after the fourth consolidation.

An allogeneic CBT was performed in March 2007. The CB unit was co-infused with haploidentical selected CD34+ SC from an unrelated donor. Conditioning was myeloablative and included fractionated TBI (total dose $1000 \mathrm{cGy}$ ), fludarabine $120 \mathrm{mg} / \mathrm{m}^{2}$ (30 mg/m2, days -5 to -2 ), cyclophosphamide $120 \mathrm{mg} / \mathrm{kg}$ $(60 \mathrm{mg} / \mathrm{kg}$, days -4 and -3$)$ and thymoglobulin $4 \mathrm{mg} / \mathrm{kg}$ ( $2 \mathrm{mg} / \mathrm{kg}$, days -2 and -1 ). After CBT, the patient has remained in complete remission with a normal performance status.

The patient consulted because she had been trying unsuccessfully to conceive. She was amenorrheic. A complete hormonal evaluation was performed to confirm that thyroid function was preserved and prolactin levels were normal.

After evaluation, a procedure of in vitro fertilization (IVF) of a donated oocyte using the patient's husband sperm was proposed. Two embryos were transferred to the uterus of our patient.

Results: A single pregnancy ensued which was carried out till term with no complications. The baby, a healthy $3500 \mathrm{~g}$ girl, was born at week $40+5$ after a cesarean section. Ten months later, both mother and child remain in good health.

Conclusion: Reproductive issues in young women who undergo myeloablative allogeneic SCT are especially difficult to address and solve.

Physicians must improve their practices, provide information and educational materials and facilitate the physician-patient discussion on fertility preservation. Guidelines with recommendations are available for health-care providers ${ }^{3}$. These recommendations are based on best available evidence. Successful pregnancies in this population have to be reported. To our knowledge, this is the first report showing a successful pregnancy and delivery after myeloablative cord blood allogeneic transplantation.

References: 1. Carter A, Robison LL, Francisco L et al. Prevalence of conception and pregnancy outcomes after hematopoietic cell transplantation: report from the bone 
marrow transplant survivor study. Bone Marrow Transplant (2006) 37, 1023-1029.

2. Nakajima $\mathrm{Y}$, Kuwabara $\mathrm{H}$, Kishimoto $\mathrm{K}$ et al. Successful pregnancy and delivery via in vitro fertilization with cryopreserved and thawed embryo transfer in an acute myeloid leukemia patient after allogeneic bone marrow transplantation. Int J Hematol. 2015 Apr; 101(4):417-20.

3. Loren AW, Brazauskas R, Chow EJ et al. Physician perceptions and practice patterns regarding fertility preservation in hematopoietic cell transplant recipients. Bone Marrow Transplant. 2013 Aug; 48(8):1091-7.

Disclosure of Interest: None declared.

AB47

Case of successful therapy with rituximab at patient with transplant associated Thrombotic microangiopathy hemolytic uremic syndrome after repeated allogenic hematopoietic stem cell transplantation

E. Pristanskova ${ }^{1, *}, K$. Kirgizov ${ }^{1,2}$, N. Sidorova ${ }^{1}$, V. Konstantinova ${ }^{1}$, A. Burya ${ }^{1}$, E. Skorobogatova ${ }^{1}$

${ }^{7} B M T$ Department, The Russian Children's Research Hospital, ${ }^{2}$ Dmitry Rogachev Federal Research Center of Pediatric Hematology, Oncology and Immunology, Moscow, Russian Federation

Introduction: Transplant associated Thrombotic microangiopathy hemolytic uremic syndrome (TA-TMA-HUS) is very severe and uncommon complication of HSCT.

Material (or patients) and methods: 15 y.o. boy suffered with ALL from 2003. First Allogenic HSCT in 3rd remission from fullmatched unrelated donor was performed in 2013 with usage of TCR a/b/CD19-depletion. Due to disease relapse, repeated haploidentical HSCT (from father) was performed in 2015 (in 4th complete remission). Conditioning: TBI $4 \mathrm{~Gy}, \mathrm{BU}$, Flu, Timo. GvHD prophylaxis: cyclo on $D+3,+4$, CsA from $D+8$, sirolimus from $D+33$. Donor and patient were compatible in blood group and rhesus. Patient engrafted on D+27 with a moderate transfusion dependence. GvHD skin gr. III. (D+24 - D+46). Kidneys malfunction started from $\mathrm{D}+43$ (high azotemia) followed by PLT level decreasing. First macrohematuria episode was revealed on D+56 (HB level de-greased to $5,6 \mathrm{~g} / \mathrm{dL}$ ). CMV-viremia was also revealed.

Due to revealed thrombocytopenia, LDH increasing to $2076 \mathrm{U} / \mathrm{l}$, decreasing of HPT level, sever azotemia, negative Coomb's test, ADAMTS 13 serum level 60\%, factor H Ab-66\%, C3-compl $107 \mathrm{mg} / \mathrm{dL}$, C4-compl - 17,8 mg/dL TA-TMA-HUS was revealed. 15 y.o. boy suffered ALL from 2003 received Allo-HSCT in 3 rd remission from MUD in 2013. Due to relapse, repeated haplo-HSCT was performed in 2015 (in CR). Conditioning: TBI 4 Gy, BU, Flu, Timo. GvHD prophylaxis: CPH on D $+3,+4$, $\mathrm{CsA}$ /sirolimus. Patient engrafted on $\mathrm{D}+27$. GvHD skin gr. III. Renal malfunction started from $\mathrm{D}+43$ (high azotemia) followed by PLT level decreasing. First macrohematuria episode was revealed on D+56 (HB level decreased to $5,6 \mathrm{~g} / \mathrm{dL}$ ). Due to thrombocytopenia, LDH increasing to 2076 $\mathrm{U} / \mathrm{l}$, decreasing of HPT level, sever azotemia, negative Coomb's test, ADAMTS 13 serum level $60 \%$, factor $H$ $\mathrm{Ab}-66 \%$, C3-compl $107 \mathrm{mg} / \mathrm{dL}$, C4-compl - 17,8 mg/dL TA-TMA-HUS was revealed.

Results: Patient was treated with defibrotide, DEXA/PRED and fresh frozen plasma with positive dy-namic - decreasing of $\mathrm{LDH}$, increasing of PLT and normalization of azotemia. Macrohematu-ria solved. Second episode of TA-TMA-HUS started from D+ 93 with fast decreasing of $\mathrm{HB}$ to $6,1 \mathrm{~g} / \mathrm{dL}$, $\mathrm{LDH}$ increasing to $1361 \mathrm{U} / \mathrm{l}$, progressed azotemia and hypofunction. Previous therapy (including IVIGs) was ineffective. Despite of absence of CD20+ cells in blood, Rituximab was infused at D+124 and D+130 (375 mg/sq.m.). After the first infusion positive reaction was reached - stable increasing of PLT level, decreasing of LDH level and renal function normalization. Follow-up period now - 7 months. Clinical status of the patient is normal. No signs of TA-TMA-HUS revealed.

Patient treated with defibrotide, DEXA/PRED and fresh frozen plasma with positive dynamic. Macrohematuria resolved. 2nd episode started from D+93. Previous therapy (including IVIGs) was ineffective. Despite of absence of CD20+ cells in blood, Rituximab was infused at D+124/+130 (375 mg/sq.m.) with positive reaction - stable increasing of PLT level, decreasing of $\mathrm{LDH}$ level and renal function normalization. Follow-up 7 months. Clinical status - normal. No TA-TMA-HUS revealed. Conclusion: This data showed effectiveness of Rituximab in case of TA-TMA-HUS in pediatric patient. More investigations must be done.

This data showed effectiveness of Rituximab in case of TA-TMA-HUS in pediatric patient.

Disclosure of Interest: None declared.

\section{AB48}

Incidence and Characterization of Hemorrhagic Cystitis Following Allogeneic Stem Cell Transplantation in a Public Hospital, Southern Brazil

S. Amaral ${ }^{1}$, A. Paz ${ }^{1}$, L. Rigoni ${ }^{1}$, G. Fischer ${ }^{1}$, L. E. Daudt ${ }^{1, *}$, L. Silla ${ }^{1}$ ${ }^{1}$ Hematology and BMT, Hospital de Clínicas de Porto Alegrel Universidade Federal do RGS, Porto Alegre, Brazil

Introduction: Hemorrhagic cystitis (HC) is a serious complication of Allogeneic Hematopoietic Stem Cell Transplantation (HSCT) afecting 12 to $25.5 \%$ of the patients. The early-onset form of HC develops during or until 72 hours after the conditioning regimen containing high doses of certain chemotherapy drugs such as Busulfan and especially Cyclophosphamide. Late-onset HC occurs from the third day on after HSCT and its etiology is multifactorial. Several risk factors for the late-onset form have been reported including graftversus-host disease (GVHD), unrelated donor, urotropic infections, male gender and myeloblative conditioning regimen.

Material (or patients) and methods: This study aimed to evaluate the incidence of $\mathrm{HC}$ in adult and pediatric patients undergoing Allogeneic HSCT and to identify risk factors associated with the development of $\mathrm{HC}$ in our population. Medical records were analyzed in 347 patients who underwent Allogeneic HSCT at Hospital de Clínicas, Porto Alegre, Brazil, from January 2001 to December 2014.

Results: $\mathrm{HC}$ occurred in 42 patients $(12.1 \% \mathrm{Cl}: 8.9-16 \%)$ at an average of 53.4 days after the procedure (standard deviation: 28.1 days). Only one patient developed early-onset $\mathrm{HC}$ on $\mathrm{D}+1$. Among the 41 patients who developed late-onset HC, BKV was the most common viral agent identified. $\mathrm{HC}$ developed in $12.8 \%$ of the patients treated with myeloablative conditioning and in $10.5 \%$ of the remaining patients $(P=0.704)$. Of the 197 patients with acute GVHD, 35 (17.8\%) developed HC and only 7 (4.9\%) showed HC in the absence of GVHD $(P<0.001)$. HC was also more frequent in males than females $(P=0.027)$. The median survival was 5.2 years ( $95 \% \mathrm{Cl}$ : $3.6-6.8$ years) in the group of patients who presented $\mathrm{HC}$ and 6.7 years $(95 \% \mathrm{Cl}$ : 5.9-7.5 years) in patients who did not $(P>0,5)$.

Conclusion: The incidence of $\mathrm{HC}$ in our series of patients was similar to that found in other studies. In our cohort of patients being male and having acute GVHD was a risk factor to develop HC.

Disclosure of Interest: None declared.

\section{AB49}

Frequency of human neutrophil antigens (HNA) in recipients of allogeneic hematopoietic stem cell allogeneic transplantation (HSCT) and correlation with procedure outcome

F. Pereira ${ }^{1}$, J. Neumann ${ }^{2}$, A. Paz ${ }^{3}$, L. Carvalho ${ }^{4}$, H. Tarasconi ${ }^{2}$, L. Rigoni ${ }^{3}$, G. Fischer ${ }^{3}$, M. Michalowski ${ }^{5}$, L. Silla ${ }^{3}$, L. E. Daudt ${ }^{1, *}$

${ }^{1} P P G$ Saude da Criança e Adolescente, Universidade Federal do Rio Grande do Sul, 'Transplant Immunology Laboratory." Santa Casa de Misericordia, ${ }^{3}$ Hematology and BMT, ${ }^{4}$ Unidade de Ambiente Protegido, ${ }^{5}$ Pediatric Oncology, Hospital de Clínicas de Porto Alegre/Universidade Federal do RGS, Porto Alegre, Brazil

Introduction: The hematopoietic reconstruction with allogeneic bone marrow is an established method of treatment for 
a variety of haematological, oncologic and immunologic diseases. HSCT is associated with considerable morbimortality due to factors like recurrence of underlying disease, HLA compatibility between donor and recipient and type conditioning regime. This study investigated the association between human neutrophil alloantigens (HNA) and engraftment, the occurrence of acute GVHD and transplant related mortality in patients who underwent allogeneic hematopoietic stem cell transplantation at our center.

Material (or patients) and methods: In a prospective cohort study carried out at Hospital de Clínicas de Porto Alegre we evaluated 28 patients transplanted between May of 2013 and April of 2014 and their respective donors. HNA typing was performed by PCR-SSP and antiHNA antibodies were detected using the LABSCREEN MULTI kit (LSMUTR-One Lambda).

Results: The age ranged from 1 to 63 years, with an average of $20.4 \pm 17.5$ years. Nineteen were pediatric patients $(<21$ years) with an average age of $10.05 \pm 6.4$ years, and among adult patients the average was $42.2 \pm 12.6$ years. There was a discreet male prevalence, $16(57,1 \%)$. The acute leukemias were frequent in 19 (67.95\%) of patients, other malignant oncohematological diseases (Hodgkin Lymphoma and nonHodgkin's Lymphoma) in $3(10.7 \%)$ and non-malignant (myelodysplastic syndrome, osteopetrosis, paroxysmal nocturnal hemoglobinuria, aplasia and granulomatous disease) in 6 (21.4\%). 19 of the patients (67.9\%), had the disease for less than 12 months at the time of the transplant and $25(89.3 \%)$ were fully HLA compatible with their donors. Myeloablative conditioning regimen was used in $16(57.1 \%)$ of the patients and the standard prophylaxis for GVHD (cyclosporine and methotrexate) was used in $15(53.6 \%)$ of the patients. The engraftment occurred at a median of 19 days. Four patients (14.3) died before the engraftment. Approximately 61\% (17 patients) showed acute GVHD (in all stages) and the rate of related mortality (TRM) was approximately $43 \%$ (12 patients). Patients who received HSCT from a related donor had TRM of approximately $41 \%$ (7 patients) and those who have received from unrelated donor had approximately $45 \%$ (5 patients). The frequency of HNA antigens detected in donors was $46,4 \%$ HNA-1a, 89,3\% HNA-1b, 3,6\% HNA-1c, 96,4\% HNA- 3a, 32,1\% HNA-3b, 96,4\% HNA-4a, 21,4\% HNA-4b, 85,7\% HNA-5a and $71,4 \%$ HNA-5b. $19 / 27$ (70\%) has a anti-HNA detected. The frequency of antibodies anti-HNA1a, anti-HNA1b, anti-HNA1c and anti-HNA2 at D0 were respectively $46,4 \%, 42,9 \%$, $42,9 \%$ and $53,6 \%$. The association between the HNA donor typing and anti-HNA antibodies of patients with day of engraftment, acute GVHD and TRM showed no statistically significant correlation.

Conclusion: The HNA frequency found in donors is as described by the literature. However, the frequency of antiHNAs antibodies was quite high in the study population, even if the majority presented the disease for less than 12 months before the transplant. Although we don't find a correlation, further studies are needed to better assess the role of the HNA on the outcome of the HSCT.

Disclosure of Interest: None declared.

\section{AB50}

Oxidative stress and anti-oxidant capacity in

hematopoietic stem cell transplantation performed children

M. Karakukcu ${ }^{1, *}$, Ç. Karakukcu ${ }^{2}$, E. Ünal ${ }^{1}$, A. Özcan ${ }^{1}$, G. Uçan ${ }^{1}$, T. Patıroğlu', M. A. Özdemir ${ }^{7}$

${ }^{7}$ Pediatric Hematology/Oncology and BMT Unit, Erciyes University Faculty of Medicine, ${ }^{2}$ Department of Biochemistry, Education and Research Hospital, KAYSERI, Turkey

Introduction: Hematopoietic stem cell transplantation (HSCT) is the curative treatment option in most malign and nonmalign diseases. Chemotherapy and radiotherapy used in conditioning regimen can lead free radical increase and oxidative stress. In this study we investigated oxidative stress

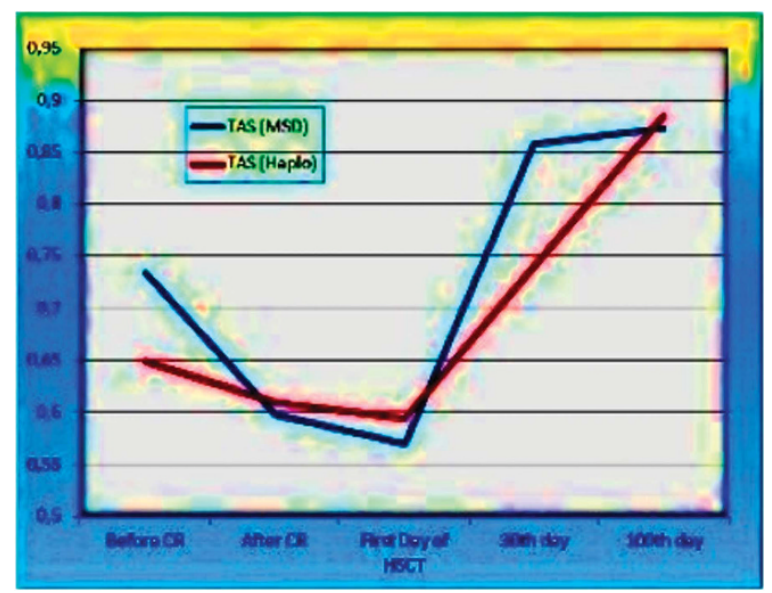

and anti-oxidant capacity in pediatric patients performed with HSCT.

Material (or patients) and methods: We included 39 pediatric patients performed with HSCT in the study. 20 of patients were performed allogeneic HSCT from siblings and 19 of patients haploidentical HSCT. The blood samples were collected five times from both groups (MSD and Haplo) before and after conditioning regimen, and first, 30th and 100th days after HSCT. Total antioxidant status (TAS), total oxidative status (TOS) and oxidative stress index (OSI) were measured and calculated for each day in both groups by spectrophotometric methods.

Results: TAS results of the patients with MSD and Haplo groups before and after conditioning regimen, and first, 30th and 100th days are shown in Figure 1.

In both groups, TAS decreased after conditioning regimen and then increased on 30th and 100th days after HSCT. But, TOS levels were not significantly different according to baseline, after conditioning regimen and HSCT. Moreover, while TAS levels did not differ between MSD and Haplo groups, TOS levels were lower in Haplo group.

Conclusion: Drugs used in conditioning regimenlead to a decrease in anti-oxidant capacity and HSCT improves oxidative status.

Disclosure of Interest: None declared.

\section{AB51}

Ventilator-dependent Takotsubo cardiomyopathy after allogeneic stem cell transplantation

N. Winkelmann ${ }^{1, *}$, J. Frietsch ${ }^{1}$, M. Fritzenwanger ${ }^{2}$, R. Pfeifer ${ }^{2}$, A. Hochhaus ${ }^{1}$, I. Hilgendorf ${ }^{1}$

${ }^{7}$ Hematology/Oncology, ${ }^{2}$ Cardiology/Pneumology, Jena University Hospital, Jena, Germany

Introduction: Cardiac failure is a known complication after hematopoietic stem cell transplantation (HSCT), but predominantly occurs in predisposed patients with low output fractions or valvular heart disease. Hereby, we present a case of acute Takotsubo cardiomyopathy in a patient without preexisting cardiac comorbidities resulting in cardiogenic shock and ventilator therapy on day +1 after allogeneic HSCT. To our knowledge this is the first report of Takotsubo cardiomyopathy after HSCT.

Material (or patients) and methods: The 62 year old female was diagnosed de novo AML M0. After induction therapy (cytarabine, mitoxantrone), 38\% blasts persisted in bone marrow on day 15 . Re-induction therapy with mitoxantrone, cytarabine and fludarabine lead to a clearance of blasts. Bridging therapy to HSCT with 5 cycles of decitabine was performed with no signs of AML recurrence. Apart from mild hypertension treated with beta blockers and AT1 antagonists, the patient had no history of cardiopulmonary disease. After 
reduced-toxicity conditioning therapy with treosulfan, fludarabine and ATG, the patient received $6,9 \times 10 \mathrm{e} 6 \mathrm{CD} 34$ positive cells per kg bw from an HLA 10/10 matched unrelated male donor without any acute distress during re-transfusion of stem cells. However, eleven hours after transplant, she complained of acute chest pain, hypotension and dyspnea with decreased oxygen saturation that was refractory to non- invasive ventilation and was immediately transferred to the ICU in cardiogenic shock.

Results: The ECG showed a heart rate of $136 / \mathrm{min}$ and an R-peak reduction from V1 to V3 without ST segment changes. High sensitive troponine, brain natriuretic peptide and creatinine kinase were elevated, but there were no signs of hemolysis. Coronary angiography was performed immediately after intubation. On examination there were no signs of coronary artery disease, but ventriculography and subsequent echocardiography revealed severe hypokinesia of the apical and medial walls (output fraction 30\%) consistent with Takotsubo cardiomyopathy. The patient received intermittent vasopressor therapy and continuous dialysis with volume restriction. She was stabilized and extubated after 8 days. Echocardiographic follow up three weeks after transplant showed completely normalized ejection fraction. The patient was discharged with normal output fraction and is well and alive on day 135 after HSCT.

Conclusion: Takotsubo cardiomyopathy is an infrequent cause of acute coronary syndrome and can be induced by physical and emotional triggers. In describing this case, we wish to remind physicans to take into account Takotsubo cardiomyopathy as a rare differential diagnosis in HSCT recipients with acute cardiac failure.

Disclosure of Interest: None declared.

AB52

Efficacy of triple drug combination in preventing nausea and vomiting $s$ after high-dose chemotherapy

P. K. Rzepecki ${ }^{1, *}$, W. Pielichowski ${ }^{1}$, K. Gawronski ${ }^{1}$, B. Mlot ${ }^{2}$, P. RZEPECKI

${ }^{1}$ Department of Internal Medicine and Haematology, ${ }^{2}$ Department of Oncology, Military Inst. of Medicine, Warsaw, Poland

Introduction: Patients frequently cite nausea and vomiting as one of the most distressing and debilitating side effects of chemotherapy. Chemotherapy-induced nausea and vomiting (CINV) can be divided into acute (24 or fewer hours after chemotherapy), delayed (more than 24 hours after chemotherapy). This distinction is made because acute CINV is believed to be mediated through serotonin receptor stimulation while delayed CINV is thought to involve multiple neurotransmitters, including opioid and neurokinin receptors. Chemotherapeutic agents have variable emetogenic potential that is affected by dose and method of administration. High-dose chemotherapy (HDC), often combined with TBI or total nodal irradiation of a varied amount, and stem cell rescue is a treatment modality applied to a wide variety of medical conditions. The delivery of high-dose therapy is almost always associated with a great degree of nausea and vomiting.

Material (or patients) and methods: A clinical study of triple drugs combination (aprepitant+palonosetron+dexamethasone) was carried out to evaluate its efficacy in preventing both acute and delayed emesis after high-dose chemotherapy (HDC):

1) BEAM \{carmustine+etoposide+cytarabine+melphalan\}

2) Mel200 \{Melphalan $200 \mathrm{mg} / \mathrm{m}^{2}$

3) CarboPe \{carboplatin+etoposide\}

4) BuCTX \{busulphan+cyclophosphamide\}

140 patients suffered from various hematological malignancies were evaluated (70 in examinated group and 70 in control group), including BEAM - 25 each, BuCTX - 15 each, Mel20020 each and CarboPe -10 each patients. The patients groups were comparable for statistical analysis in terms of number of patients, their age, sex, weight and underlying diseases. Historical control groups of patients were treated with dexamethasone (dex) and palonosetron. The observation period started with the initiation of chemotherapy $(0 \mathrm{~h})$ and continued for $24 \mathrm{~h}$ after completion of the chemotherapy for acute phase, and during five days after finishing chemotherapy for delayed phase. The response rate of the study drugs was evaluated by the following 4-grade scale based on the condition of nausea and vomiting: highly, moderately or slightly effective and not effective.

Results: The best results were observed (triple drugs combination) in the early phase after BEAM conditioning and in delayed phase after Mel200 high-dose chemotherapy.

Conclusion: Triple drugs combination was more effective than palonosetron (+ dex) treatment in preventing CINV after high-dose chemotherapy before haematopoietic stem cell transplantation.

References: Schmitt T, Goldschmidt H, Neben K, i wsp. Aprepitant, granisetron, and dexamethasone for prevention of chemotherapy-induced nausea and vomiting after highdose melphalan in autologous transplantation for multiple myeloma: results of a randomized, placebo-controlled phase III trial. J Clin Oncol. 2014; 32: 3413-3420.

Stiff PJ, Fox-Geiman MP, Kiley K, i wsp. Prevention of nausea and vomiting associated with stem cell transplant: results of a prospective, randomized trial of aprepitant used with highly emetogenic preparative regimens. Biol Blood Marrow Transplant. 2013; 19: 49-55.

Mikus G, Burhenne J, Ho AD, Egerer G. Prevention of nausea and vomiting associated with stem cell transplant: results of a prospective, randomized trial of aprepitant used with highly emetogenic preparative regimens. J Clin Oncol. 2014; 32: 3413-3420.

Disclosure of Interest: None declared.

\section{AB53}

Graft failure following allogeneic hematopoietic stem cell transplantation: our experience

R. Rady ${ }^{1, *}$, A. Abdelkader ${ }^{1}$, M. abdelsalam ${ }^{1}$, M. abdelmohsen ${ }^{1}$, M. ragaay ${ }^{7}$, M. saad ${ }^{1}$, D. abdelaziz ${ }^{2}$, M. El Ghamrawy ${ }^{\prime}$, K. Salama

${ }^{1}$ department of pediatrics, hematology and BMT unit, ${ }^{2}$ department of pediatrics, faculty of medicine, Cairo University, Cairo, Egypt

Introduction: Graft failure is considered an important cause for morbidity and mortality following allogeneic HSCT. (Remberger $M$, et al. 2011).

Material (or patients) and methods: Twenty two fully HLAmatched, allogeneic bone marrow transplants for non malignant diseases have been performed from 2012 to 2015 at BMT Unit in Cairo University Children Hospital. One HLAmatched allogeneic BMT performed back in 2008. Thalassemia major (TM) forms $56.5 \%(n=13)$ of total transplants so far, SAA contributes to $34.7 \%(n=8)$ of total transplants while severe combined immunodeficiency (SCID) forms $8.69 \%(n=2)$ of total transplants.

Results: Seven patients suffered from graft failure (30.4\%). Four patients were classified as primary graft failure $(57.1 \%)$ while three cases $(42.8 \%)$ were classified as secondary graft failure and they were secondary to acute graft versus host disease (aGVHD), CMV reactivation and non compliance to post transplant immunosuppressives respectively. Six patients (one male and five females) were cases of TM; received Busulfan (Bu)-cyclophosphamide (Cy)- antithymocyte globulin (ATG) as a conditioning regime and cyclosporin (CSA) as a prophylaxis for GVHD. While one patient was a case of SAA, male, received CY-Flu (fludarabine)-ATG as a conditioning regimen and CSA as prophylaxis for GVHD. Donors of stem cells in the seven cases were males. Four patients received peripheral stem cells (PSC) and three patients received bone marrow harvest in their $1^{\text {st }}$ transplant. Three patients were retransplanted using ATG ( $10 \mathrm{mg} / \mathrm{kg} /$ day) for 3 days and one patient received TBI (total body irradiation) - CY- FLU in retransplant. Outcome of graft failure was death in one (14.2\%) 
case, autologous recovery in three $(42.8 \%)$ cases, engraftment post re-transplant in three $(42.8 \%)$ cases. Two cases showed morbidity post engraftment; post retransplant. Graft failure frequency; one case in 2008, five cases in 2012-2013 and one case in 2014 and none in 2015.

Conclusion: Our regimen has some limitations; we use oral busulfan with no feasibility for monitoring drug level. Challenges are being faced in our center over the past 3 years and continuous re-evaluation and assessment of our practice.

References: Remberger M, Mattsson J, Olsson R, Ringden $\mathrm{O}$. Second allogeneic hematopoietic stem cell transplantation (HSCT): a treatment for graft failure. Clin Transplant 2011; 25: E68-E76.

Disclosure of Interest: None declared.

\section{AB54}

Early hospital readmitance in post autologous

hematopoietic stem cell transplantation patients - a single center experience

R. Tenreiro ${ }^{1, *}$, M. Santos ${ }^{1}$, M. Pereira ${ }^{1}$, R. Guilherme ${ }^{1}$, P. César ${ }^{1}$, M. Gomes", J. P. Carda ${ }^{1}$, E. Cortesão ${ }^{1}$, A. I. Espadana ${ }^{\prime}$, C. Geraldes', L. Ribeiro ${ }^{1}$

${ }^{1}$ Cento Hospitalar e Universitario de Coimbra, Coimbra, Portugal

Introduction: High-dose therapy with autologous stem cell transplantation (ASCT) has been considered a standard frontline treatment for younger multiple myeloma patients (MM) with adequate organ function; although several studies on safety are available, few reports focus on immediate complications following ASCT.

Our aim is to evaluate the early complications requiring hospital re-admission in the first 100 days following ASCT in MM patients and its relationship with age and survival.

Material (or patients) and methods: We conducted a retrospective analysis of patients with $\mathrm{MM}$ who underwent ASCT between 01-01-2007 and 31-12-2014, who were readmitted in the first 100 days post-ACST. Survival was calculated according to Kaplan-Meier and Log-rank methods.

Results: In these 8 years, $161 \mathrm{MM}$ patients underwent ASCT, with a median age of 58.6 years (range 28-77years) and a Male/Female ratio of 1.33. High-dose melphalan conditioning regimen was used, followed by ASCT. Half of the patients were discharged in the 20 days after ASCT (range 8-108 days). Median survival was not yet achieved in this population (median follow-up 4.2 years).

In the first 100 days post ASCT there were 22 hospital admissions corresponding to 18 patients $(11,2 \%)$. Median hospital stay was 9 days (range 1-68 days). The majority of those were due to infectious complications $(n=10)$, renal insufficiency $(n=4)$, thrombotic events $(n=2)$, gastro-intestinal (GI) bleeding $(n=1)$ and other causes $(n=5)$. The main site of infection was $\mathrm{Gl}$ tract $(n=5)$, followed by respiratory $(n=3)$ and urinary tract $(n=1)$ infections. Only in 1 case (Gl infection) was an infectious agent identified - E. faecium. There was 1 death registered in this group of patients, attributed to disease progression. Patients with hospital admissions in the first 100 days post ASCT had shorter median survival (7,5 months) than those who were not admitted $(P=0,041)$.

There was no statistical significant difference when comparing age (under or above 65 years), the number of previous lines of treatment, melphalan dosage $\left(200 \mathrm{mg} / \mathrm{m}^{2}\right.$ vs $140 \mathrm{mg} / \mathrm{m}^{2}$ or the number of mobilizations required. These factors were also not able to predict survival in our population.

Conclusion: ASCT is widely used in MM patients, and is normally well tolerated, however a small proportion of patients require early hospital readmission within the first 100 days. In our group of patients this was the only factor associated with reduced survival.

Disclosure of Interest: None declared.
AB55

"A pilot study on the efficacy of Lactobacillus brevis CD2 lozenges in preventing oral mucositis by high-dose chemotherapy with autologous hematopoietic stem cell transplantation"

S. Giammarco ${ }^{1, *}$, A. Di Giovanni ${ }^{1}$, E. Metafuni ${ }^{1}$, P. Chiusolo ${ }^{1}$, S. Sica ${ }^{1}$

${ }^{1}$ Hematology Institution, Università Cattolica del Sacro Cuore, Rome, Italy

Introduction: Treatment-induced oral mucositis is one of the most debilitating side effects from the patient's perspective and profoundly influencing QoL. OM is a pathological process characterized by mucosal damage, ranging from mild inflammation to deep ulcerations and affecting one or more parts of the alimentary tract, from the mouth to the anus, as a consequence of chemotherapy and/or radiation therapy. (1) OM is associated with pain, bleeding, dysphagia, infections and food intake impairment, which can limit adequate nutritional intake. $\mathrm{OM}$ is strongly associated with bacteremia and sepsis due to E. Coli, P. Aeruginosa, and Candida. Two major events lead to OM; one direct injury caused by the ablative regimen, and local infections that develop on the damaged mucosal surface, which are exacerbated by neutropenia. Treatment and prevention of OM are palliative and the efficacy of most of the measures used have not been clearly established. Prophylactic measures employed are use of: chlorhexidine, soda bicarbonate rinses, acyclovir and ice. For treatment of $\mathrm{OM}$ and its associated pain local anesthetic, diphenhydramine, nystatin, or sucralfate are used alone or in combination as mouthwash. Several studies showed the successful treatment of OM with the strain of Lactobacillus brevis, due to the process of microflora manipulation. (2) The strain $L$. brevis produces high levels of the enzymes arginine deaminase and sphingomyelinase. Arginine deaminase converts arginine to ammonia and citrulline, reducing the amount of available arginine to be converted to nitric oxide, a major mediator of inflammation. Sphingomyelinase can hydrolyze platelet activating factor, a potent inflammatory cytokine, known to be associated with oral mucositis in radiation therapy.

Material (or patients) and methods: The aim is to assess if the probiotic $L$. brevis $C D 2$ lozenges can reduce the incidence and severity of OM in patients undergoing HSCT. We enrolled 16 patients (pts), affected by multiple myeloma and submitted to autologous HSCT at our division between March 2014 and February 2015. Eight pts received $6 \mathrm{~L}$. brevis CD2 lozenges

\begin{tabular}{|c|c|c|}
\hline & ARM with L.Brevis & Control ARM \\
\hline Patients & 8 & 8 \\
\hline$M / F$ & $4 / 4$ & $4 / 4$ \\
\hline Median Age(range) & 62 y $(51-66)$ & 61 y $(51-64)$ \\
\hline Disease & $8 \mathrm{MM}$ & $8 \mathrm{MM}$ \\
\hline $\begin{array}{l}\text { Conditioning } \\
\text { Regimen }\end{array}$ & HD-Melfalan & HD- Melfalan \\
\hline Median CD34+ dose & $6 \times 10^{6} / \mathrm{kg}(2.9-8)$ & $5.7 \times 10^{5} / \mathrm{kg}(3.4-7.2)$ \\
\hline $\begin{array}{l}\text { Median Neutrophil } \\
\text { recovery }\end{array}$ & $11.5 \mathrm{~d}(9-24)$ & $11.5 \mathrm{~d}(11-13)$ \\
\hline $\begin{array}{l}\text { Median Platelets } \\
\text { recovery }\end{array}$ & $12 d(8-26)$ & $12.5 \mathrm{~d}(11-14)$ \\
\hline FUO & $3 / 8$ & $5 / 8$ \\
\hline Sepsis & $1 / 8$ & $1 / 8$ \\
\hline OM grade I & 4 & 2 \\
\hline OM grade II & 2 & 4 \\
\hline OM grade III & 1 & 2 \\
\hline
\end{tabular}


per day, from 14 days prior chemotherapy and till resolution of $\mathrm{OM}$ and reported all symptoms on a daily diary. The remaining 8 patients received standard OM prevention including chlorhexidine, saline rinses and nystatin.

Results: Patients characteristics are summarized on TABLE 1. Fourteen out 16 pts developed diarrhea: stool cultures were positive for Candida in 2 pts and Clostridium difficile in 1 pt; $7 / 16$ pts developed FUO and 2 sepsis. All pts developed OM with different grade: (grade I: 6; grade II:6; grade III:3; NA:1). One patient died during pancytopenia period for respiratory failure due to H1N1 influenza. Although there was a trend in a reduction of grade II and III OM, we found no statistical difference between the two arms.

Conclusion: No differences were observed in terms of engraftment and complications post HSCT. In this preliminary experience the use of Lactobacillus brevis was safe and well tolerated. References: 1. Niscola $P$ et al. Mucositis in patients with hematologic malignancies: an overview. Haematologica. 2007 Feb;92.

Sharma A et al. Lactobacillus brevis CD2 lozenges reduce radiation-and chemotherapy-induced mucositis in patients with head and neck cancer: A randomized double-blind placebo-controlled study. Eur J Cancer.

Disclosure of Interest: None declared.

AB56

Evaluation of short term ruxolitinib tapering strategy prior to allogeneic stem cell transplantation for primary myelofibrosis through the transition of serum cytokines and growth factors; North Japan Hematology Study Group S. Shiratori ${ }^{1,2,{ }^{*}, T \text {. Tateno }}{ }^{1}$, S. Ito ${ }^{1}, Y$. Tsutsumi ${ }^{1}$, T. Teshima ${ }^{2}$ on behalf of North Japan Hematology Study Group

${ }^{1}$ Hematology, Hakodate Municipal Hospital, Hakodate, Hokkaido, Japan, Hakodate, ${ }^{2}$ Hematology, Hokkaido University Graduate School of Medicine, Sapporo, Japan

Introduction: Safety of the discontinuation of Ruxolitinib (Ruxo), a JAK1/2 inhibitor, prior to allogeneic hematopoietic stem cell transplantation (allo-HSCT) for myelofibrosis (MF) is still controversial. "JAK ALLO Study" reported serious adverse events in early phase during allo-HSCT, indicating a rapid disease progression or withdrawal symptom by the discontinuation of Ruxo, ${ }^{1}$ while such events were not described in several retrospective studies. Furthermore no previous study evaluated the safety of the discontinuation of Ruxo prior to allo-HSCT in association with changes in serum levels of cytokines or growth factors.

Material (or patients) and methods: We conducted a pilot study in two patients with primary MF treated with Ruxo prior to allo-HSCT. Ruxo was tapered off 6 days before the initiation of reduced intensity conditioning regimen using fludarabine $\left(180 \mathrm{mg} / \mathrm{m}^{2}\right)$, intravenous busulfan $(9.6 \mathrm{mg} / \mathrm{kg})$ and $4 \mathrm{~Gy}$ total body irradiation. Primary endpoints were the following: 1) Engraftment and survival at 4 weeks after allo-HSCT, 2) Analysis of serum cytokines and growth factors before and after Ruxo and during allo-HSCT. This study was approved by the Ethics Board in our hospital and the study protocol is registered in University Hospital Medical Information Network Clinical Trials Registry (UMIN000019421).

Results: Both patients improved splenomegaly after the administration of Ruxo and no disease progression or withdrawal symptom was developed after the discontinuation of Ruxo (Figure), and achieved engraftment with complete donor chimerism by day 28 after allo-HSCT. Serum levels of VEGF, IL- 6 and SIL-2 R were decreased after the administration of Ruxo. However, those of IL-6, sIL-2 R and MCP-1 were significantly increased after the discontinuation of Ruxo. IL-6 and SIL-2 R persisted higher serum levels until day 28 after allo-HSCT, while those of MCP-1 was decreased to baseline on day 28. Serum level of VEGF was also slightly increased after the discontinuation of Ruxo; however, the elevation was temporary and showed a stable transition during allo-HSCT (Figure).

Conclusion: Our preliminary study suggests that Ruxo tapering strategy 6 days prior to initiation of conditioning regimen is safe without causing withdrawal symptom of Ruxo. Serum level of VEGF might reflect disease status of MF possibly unaffected by engraftment or GVHD during allo-HSCT, although these results need to be validated in a larger study. References: 1. Robin M, Francois S, Huynh A, Cassinat B, Bay $\mathrm{J}-\mathrm{O}$, Cornillon J et al. Ruxolitinib before allogeneic hematopoietic stem cell transplantation (HSCT) in patients with myelofibrosis: a preliminary descriptive report of the JAK

[AB56]
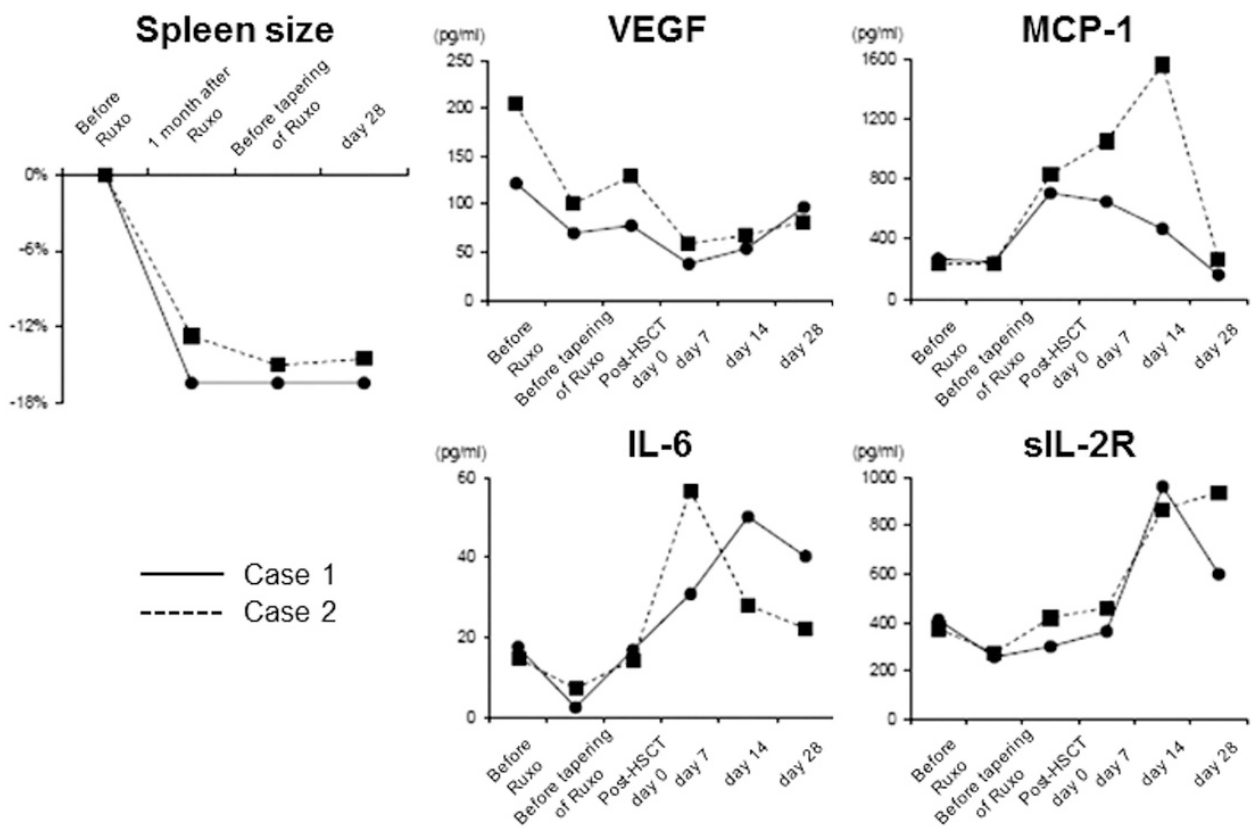
ALLO Study, a phase II trial sponsored by Goelams-FIM in collaboration with the Sfgmtc. Blood 2013; 122: 306.

Disclosure of Interest: None declared.

\section{AB57}

Endothelial cell damage in idiopathic pneumonia syndrome following haematopoietic stem cell transplantation for primary immunodeficiency T. Altmann 1,*, J. Slack ${ }^{1}$, M. Slatter ${ }^{1}$, C. O'Brien ${ }^{2}$, A. Cant ${ }^{1}$, M. Thomas ${ }^{1}$, M. Brodlie ${ }^{1}$, S. Annavarapu ${ }^{2}$, A. Gennery $^{1}$

${ }^{1}$ Institute of Cellular Medicine, Newcastle University, ${ }^{2}$ Great North Children's Hospital, Royal Victoria Infirmary, Newcastle upon Tyne, United Kingdom

Introduction: Idiopathic pneumonia syndrome (IPS) post haematopoietic stem cell transplantation (HSCT) is a rare complication, difficult to treat, with a poor prognosis and often fatal. ${ }^{1,2}$ The aetiology remains disputed, but conditioning, graft versus host disease (GvHD) and occult infection have been implicated. Recent murine studies implicate endothelial cell (EC) activation and injury. ${ }^{2}$ as demonstrated by endothelial leak of fibrin, endothelial apoptosis, veno-occlusive disease (VOD), venous embolization and fibrin deposition, pulmonary hypertension $(\mathrm{PH})$, and pulmonary vascular intimal hyperproliferation on histopathological specimens. ${ }^{2,3}$ Historically, few lung biopsies were performed on our patients with IPS Results have been variable, with no underlying disease process described. We retrospectively examined lung biopsies in a primary immunodeficiency (PID) paediatric HSCT cohort, specifically looking for EC activation/injury.

Material (or patients) and methods: PID patients who underwent HSCT between 01/2000-07/2015 and had a postHSCT lung biopsy were identified from the pathology database. Patient records were reviewed and lung biopsies re-examined for signs of EC activation/injury.

Results: 313 PID patients underwent HSCT, 9 were identified as IPS with 8 having biopsy material available. All presented with respiratory distress. 6 received pre-HSCT conditioning. Median time of symptom onset D+11 post-HSCT (range D 1 of conditioning - D+36 post-HSCT), 2 had symptoms from birth. 8 required mechanical ventilation. 2 received Defibrotide (DF) for VOD. Mortality was $88 \%$. Histology: None had signs of viral, bacterial or fungal infection. 5 had widespread capillary leak of fibrin in alveolar spaces, no patients showed evidence of active VOD, 4 had arterial myointimal thickening, and therefore $\mathrm{PH}, 2$ of which had no alveolar capillary fibrin leak. 2 had Bronchiolitis Obliterans (BO). 1 had fibrin deposition, intraepithelial T cells and atypical mildly reactive pneumocytes. At post mortem, 1 patient had intra-alveolar fibrin, VOD, $\mathrm{PH}$, intraepithelial T cells and BO. 1 patient had rhinovirus identified from bronchoalveolar lavage but had no reactive inflammatory cells. No micro-organisms were identified from other patients. 4 had ground glass appearances on Chest X-Ray/CT, 3 had non-specific consolidation, 1 demonstrated bilateral upper zone collapse, 1 had no radiological abnormalities.

Conclusion: Our patients with clinically and radiologically unexplained respiratory symptoms, designated IPS, all show underlying disease processes associated with EC damage, the cause of which remains unclear. Possible factors include previous infection, conditioning agents or the underlying PID with inflammation. Further work, using cellular markers for EC activation and damage, is needed to confirm EC damage in these biopsies. DF is an agent that modulates EC damage ${ }^{2}$ and may be useful in the treatment of these patients, although stratifying which patients will benefit from DF requires further study.

References: 1. Khurshid I et al. Postgrad Med J 2002;257.

2. Cooke KR et al. Biol Blood Marrow Transplant 2008;23.

3. Budhiraja R et al. Circulation 2004;159.

Disclosure of Interest: None declared.

\section{AB58}

Bilateral chylothorax: Which is to blame?

U. Kocak ${ }^{1, *}$, Z. Kaya ${ }^{1}$, I. Altan ${ }^{1}$, T. Sismanlar ${ }^{2}$, O. Egritas Gurkan ${ }^{3}$, A. Tapisiz ${ }^{4}$, O. Konus Boyunaga ${ }^{5}$, I. Yenicesu ${ }^{7}$

${ }^{7}$ Gazi University School of Medicine Dpt of Pediatrics Unit of BMT, ${ }^{2}$ Gazi University School of Medicine Dpt of Pediatrics Unit of Pulmonology, ${ }^{3}$ Gazi University School of Medicine Dpt of Pediatrics Unit of Gastroenterology, ${ }^{4}$ Gazi University School of Medicine Dpt of Pediatrics Unit of Infectious Diseases, ${ }^{5}$ Gazi University School of Medicine Dpt of Radiology, Ankara, Turkey

Introduction: Chylothorax is lymphatic fluid leakage into pleural space with high triglyceride content and milky appearance. Etiology could be congenital, trauma, malignancy or granulomatous infections. This report describes multifactorial etiology of bilateral chylothorax in a child after stem cell transplantation (SCT).

Material (or patients) and methods: A 16 years old male with monosomy 7 positive refractory AML had undergone SCT from a matched related donor with total body irradiation based conditioning and secondary prophylaxis with voriconazole.

Results: At 3 months posttransplant with 100\% donor chimerism and monosomy negativity he had dysphagia, vomiting, anal pain and fever which prompted ampirical antibiotics. On the second day facial angioedemateous like swelling, resistant to steroids and antihistaminics was noticed with no sign of extramedullary relapse in paranasal and neck CT. On the 7th day he developed diarrhea. Although rectal biopsy proved severe intestinal GVHD no therapy was given as diarrhea resolved. Fifteen days later liver function tests increased and biopsy verified GVHD that lead to tacrolimus and steroid treatment. While performing liver biopsy milky appearing fluid was noticed. Abdominal US showed perihepatic fluid $(6 \mathrm{~mm})$. Invasive aspergillosis history and newly developed cough directed a thorax $\mathrm{CT}$ which displayed bilateral pleural ( $20 \mathrm{~mm}$ in left, $24 \mathrm{~mm}$ in right) effusion and $5 \mathrm{~mm}$ cavitary lesion in the left lung. Thoracocentesis drained chylohemorrhagic fluid with a triglyceride content of $1150 \mathrm{mg} / \mathrm{dl}$ and galactomannan and aspergillus PCR positivity. Central venous line was removed for leakage suspicion. As new lesions under voriconazole have emerged, liposomal amphotericin B (L-AMB) and fatrestricted oral diet and ocreotide were started. In the meantime although morphologically in remission monosomy 7 positivity was detected that encouraged tacrolimus withdrawal. At 1st month of ocreotide and L-AMB treatment he developed fever with increased coughing. Thorax $\mathrm{CT}$ showed ground glass appearance and enlarged effusion (52 $\mathrm{mm}$ left, $13 \mathrm{~mm}$ right). Inflammatory fluid was drained and caspofungin was added to L-AMB along with wide spectrum antibiotics. No microorganism was detected in further tests. Besides ocreotide he had TPN nil by mouth for 10 days which caused decrease in pleural effusions (12 $\mathrm{mm}$ left, $7 \mathrm{~mm}$ right). He received combined antifungal therapy for 8 weeks with resolution of the lesions. Repeated bone marrow examinations showed remission and monosomy negativity. He is now well at 16th months posttransplant with minimal pleural effusion receiving ocreotide and cyclosporine for upper GI GVHD. Conclusion: Chylothorax is an uncommon cause of pleural effusion in children. As treatment should be based on determining the underlying cause, it is of paramount importance to define the etiology. In the absence of apparent risk factors such as trauma this could be difficult. That is particularly challenging in the transplant setting since the etiology could be multifactorial as in our patient. Although the beginning was chylous ascites the bilateral nature of chylothorax precluded us from considering it being the origin. We assume that the reason of bilateral chylothorax in our patient is iatrogenic trauma by TBI as well as noniatrogenic trauma by forceful emesis and coughing associated with GVHD and aspergillus infection where all could be common in the posttransplant setting.

Disclosure of Interest: None declared. 
AB59

Total body irradiation (TBI)-based versus chemotherapybased myeloablative conditioning regimen before allogeneic stem cell transplantation (allo-sct) for acute myeloid leukemia (AML). A single centre experience A. M. Carella $a^{1,}$, , R. Angarano ${ }^{1}$, E. Merla ${ }^{1}$, M. M. Greco ${ }^{1}$, L. Savino ${ }^{7}$, M. Troiano ${ }^{2}$, S. Parisi ${ }^{2}$, N. Cascavilla ${ }^{\prime}$

${ }^{1}$ Heamatology and Bone Marrow transplant unit, ${ }^{2}$ Radiotherapy Service, IRCCS "Casa Sollievo della Sofferenza", San Giovanni Rotondo (Foggia), Italy

Introduction: Cyclophosphamide combined with $\mathrm{TBI}$ or busulfan are the most widely used myeloablative conditioning regimens in patients with AML. Recent data regarding their comparative effectiveness are lacking.

Material (or patients) and methods: In this study we retrospectively assessed the outcome of 103 patients with AML in first or second complete remission $(n=70)$ and refractory disease $(n=33)$ who received myeloablative conditioning either with a TBI-based $(n=47)$ or chemotherapybased $(n=56)$ regimen for SCT from an HLA-matched sibling $(n=56)$ or an unrelated donor $(n=28)$ or a haploidentical donor $(n=19)$. The conditioning regimen sequence was compared according to overall survival (OS), leukemia-free survival (LFS), TRM, leukemia relapse and GVHD.

Results: Overall survival of all patients was $50 \%$ at 3 years. According to disease status, 3 years probabilities of OS were $64 \%$ in CR and $22 \%$ in refractory disease at time of transplant $(P<0.0001)$. Three-year probabilities of survival were $59 \%$ and $43 \%$ for TBI and chemotherapy $(P=0.1)$. Three-years probabilities of DFS were $39.5 \%$ and $20.5 \%$ for TBI and chemotherapy $(P=0.3)$. In multivariate analysis for OS, the indipendent factors statistically significant were disease status at transplant and GvHD grade III-IV. The cumulative incidence of acute GvHD were $38 \%$ in TBI arm and $61 \%$ in chemotherapy arm $(P=0.02)$. Corresponding incidences of transplant-related mortality (TRM) were $10 \%$ and $16 \%$ respectively in patients receiving $\mathrm{TBI}$ compared chemotherapy.

Conclusion: In conclusion in our experience TBI is associated with superior outcomes compared with chemotherapy in patients with $A M L$, an important result with the use of TBIbased conditioning regimen is the less development of acute GVHD (38\%) respect to chemotherapy-based (61\%).

Disclosure of Interest: None declared.

\section{AB60 \\ BAM instead of BEAM in lympholld heamatologic malignancies autografted? \\ B. Marcq ${ }^{1, *}$, N. Contentin ${ }^{1}, H$. Tilly ${ }^{7}$ \\ ${ }^{1}$ Centre Henri Becquerel, Rouen, France}

Introduction: Autologous stem cell therapy, with BEAM conditioning regimen is a treatment phase of "slow responders" diffuse large cell lymphomas, early relapsed /refractory Hodgkin disease or young mantle cells lymphomas patients. Because of breakdown BCNU (carmustine) manufacturing in November 2013, we replaced the BEAM conditioning regimen (Bicnu $300 \mathrm{mg} / \mathrm{m}^{2}$ D-6, Etoposide $200 \mathrm{mg} / \mathrm{m}^{2}$ D-6 to D-3, Cytarabine $400 \mathrm{mg} / \mathrm{m}^{2}$ D- 6 to D-3, Melphalan $140 \mathrm{mg} / \mathrm{m}^{2}$ in $\mathrm{J}-2$ ) by a BAM one (Cytarabine $1500 \mathrm{mg} / \mathrm{m}^{2}$ D-4 to D-3, Melphalan $140 \mathrm{mg} / \mathrm{m}^{2}$ D-2, Busilvex $3.2 \mathrm{mg} / \mathrm{kg}$ D-7 to D-5). This is equivalent to replace Bicnu by the Busilvex and increases the dose of $46.67 \%$ Aracytine.

Material (or patients) and methods: From January 2014 to June 2015, we retrospectively analyzed toxicities of these two regimens: BEAM versus BAM in a paired study, including 29 patients treated with BEAM and 21 with BAM.

The average patients' age at D0 was 50 years (in BEAM group) and 51 years (in BAM one). There were no significant differences between the two groups regarding the stage of the disease. Most of the patients were followed for NHL (16 $\mathrm{NHL}$ and $5 \mathrm{MDH}$ in the BAM group, $20 \mathrm{NHL}$ and $9 \mathrm{MDH}$ in the BEAM one).
Results: Study of the short-term toxicities reveals that conditioning BAM is more toxic considering platelets reconstitution with a median of 14 days for the BAM versus 4.5 days for BEAM $(P=0.013)$ for platelets above $50 \mathrm{G} / \mathrm{I}$, a median number of transfusion 8.48 versus $6.33 \mathrm{MCP}(P=0.08)$, and a higher number of patients with severe mucositis $(P=0.0001)$. Nevertheless, there is no significant difference in hospitalization duration, aplasia length, and short-term infectious complications.

Long-term toxicities will be developed, available in March 2016 with a follow-up of 1 year.

Conclusion: In conclusion, BAM conditioning regimen induces longer thrombocytopenia, higher transfusion needs and increasing risk of bleeding and transfusion complications. The gastrointestinal toxicity is increased with more frequent and more severe mucositis, promoting undernutrition. A complement of this study will be presented regarding the long-term effectiveness of this type of procedure and its delayed toxicity.

Disclosure of Interest: None declared.

\section{AB61}

Unconditioned Transplant in Severe Combined Immunodeficiency

D. Abd Elaziz,", R. El Hawary², S. Meshaal', A. Abdelkader', I. Youssry ${ }^{1}$, K. Salama ${ }^{1}$, M. Ragaay ${ }^{7}$, A. El Marsafy ${ }^{7}$

${ }^{1}$ Pediatric Department, ${ }^{2}$ Clinical and Chemical Pathology Department, Cairo University, Cairo, Egypt

Introduction: Severe combined immunodeficiencies (SCIDs) are a genetically heterogeneous group of inherited defects characterized by severe abnormalities of the immune system. The infants present in the first few months of life with severe, recurrent, and opportunistic infections.

Material (or patients) and methods: Two T'B ${ }^{-} K^{-}$SCID patients were transplanted in the Bone Marrow Transplantation (BMT) Unit in Cairo University Children Hospital on 2015, using unconditioned transplant and Cyclosporine as graft versus host disease (GVHD) prophylaxis.

Results: Patient 1: TB $^{-} \mathrm{NK}^{-}$female SCID patient diagnosed at day 4 of her life, she was the $3^{\text {rd }}$ affected and $2^{\text {nd }}$ diagnosed member in a consanguineous family. She was transplanted at 8 months old from her fully matched sister.

Pre-transplant her WBC was ranging from 1500 to 2500 cells per milliliter and the absolute lymphocytes count (ALC) was 400 cells per milliliter.

$\mathrm{T}$ and $\mathrm{B}$ cell engraftment were on $\mathrm{D}+30$ post-transplant.

D +30: TLC: 3700 ALC: $40 \%(1480)$

CD3 $^{+}:$61.3\% (907) CD3 $^{+}$CD4 $^{+}: 37.5 \%$ (555) CD3 $^{+}$CD8 $^{+}: 17.3 \%$

(256) CD3 $^{-C^{+}}$56 $^{+}: 6.1 \%$ CD19 $^{+}: 8.2 \%$

CD19 $^{+}$IgD ${ }^{+}$CD27: $85 \%$ CD19 $^{+}$IgD CD27 $^{+}:$7\% CD19 $^{+}$lgD ${ }^{+}$ $\mathrm{CD}_{27}^{+}: 2.5 \%$

CD4 $^{+}$CD $31^{+}: 16.7 \%$ CD4 $^{+}$CD $31^{+}{ }^{+}$CD45RA $^{+}: 35 \%$ CD4 $^{+}$CD45RA $^{+}: 7 \%$ The lymphocytes count was $>1500$ on day +150 posttransplant. The last IVIG transfusion was on D+38 and her $\lg \mathrm{G}$ is $>600 \mathrm{mg} / \mathrm{dl}$ with normal lgM levels.

She is now $D+335$ post-transplant doing fine without any complications or infections apart from self-clearing low viremia of CMV virus on D+243 (CMV PCR was < 1000).

Patient 2: T'B ${ }^{-} \mathrm{NK}^{-}$male SCID patient diagnosed on 1.5 months old as he was the $2^{\text {nd }}$ affected and $1^{\text {st }}$ diagnosed member in a consanguineous family. He received BCG vaccine; therefore, he received anti-tuberculous drugs. He was transplanted at 3.5 months old from his fully matched brother.

Pre-transplant his WBC was ranging from 1000 to 2500 cells per milliliter and the ALC was 150 cells per milliliter.

He was transplanted while he had severe Klebsiella pneumonia and Klebsiella bacteremia, all these infections were cleared post-transplant. He developed acute moderate skin GVHD which was treated by steroid.

$T$ and $B$ cell engraftment were on $\mathrm{D}+75$ post-transplant. The lymphocytes count was $>1500$ on day +75 post-transplant. D 75+: TLC: 11,000 ALC: $16 \%$ (1760). 
CD3 $^{+}: 69.7 \%$ (1226) CD3 $^{+}$CD4 $^{+}:$: 20.2\% (247) CD3 ${ }^{+}$CD8 $^{+}: 34.1 \%$ (418) CD3 $^{-}$CD56 $^{+}: 20.1 \%$ CD19 $^{+}: 1 \%$

CD19 $^{+}$IgD ${ }^{+}$CD27 $^{-}: 24.2 \%$ CD19 $^{+}$IgD CD27 $^{+}: 8.1 \%$ CD $19^{+}$ $\operatorname{lgD}^{+} \mathrm{CD}^{+} 7^{+}: 2 \%$.

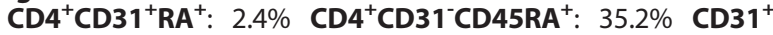
CD45RA: $1.4 \%$

The last IVIG transfusion was on D+45 and his lgG is $>600 \mathrm{mg} /$ dl with normal lgM levels.

He was readmitted in the hospital due to CMV reactivation on day +72 which was treated by using Ganciclovir, also he developed hypertension, hyperkalemia as side effects from the medications and local reaction at the site of BCG vaccine on D +80 that persist few days then resolved. The patient is now $D$ +170 post-transplant doing well.

Conclusion: These patients were the $1^{\text {st }}$ SCID babies transplanted by unconditioned transplant in our center and both have rapid good response after BMT.

Disclosure of Interest: D. Abd Elaziz Funding from: There is no conflict of interset, R. El Hawary Funding from: There is no conflict of interset, S. Meshaal Funding from: There is no conflict of interset, A. Abdelkader Funding from: There is no conflict of interset, I. Youssry Funding from: There is no conflict of interset, K. Salama: None declared, M. Ragaay Funding from: There is no conflict of interset, A. El Marsafy Funding from: There is no conflict of interset.

\section{AB62}

Treosulfan plus fludarabine - encouraging conditioning for allogeneic hematopoietic stem cell transplantation in multiple myeloma

G. Afram ${ }^{1, *}$, H. Nahi ${ }^{1}$, J. Liwing ${ }^{1}$, B. Heeg ${ }^{2}$, J. Mattsson ${ }^{3}$, K. LeBlanc ${ }^{7}$, B. Björkstrand ${ }^{i}$, G. Gahrton ${ }^{i}$, P. Ljungman ${ }^{7}$

${ }^{1}$ Hematology, Karolinska, Stockholm, Sweden, ${ }^{2}$ Epidemiology, Groningen, Groningen, Netherlands, ${ }^{3}$ CAST, Karolinska, Stockholm, Sweden

Introduction: Allogeneic transplantation (Allo-SCT) in multiple myeloma is controversial mainly due to the relatively high non-relapse mortality (NRM). Treosulfan is a prodrug of a bifunctional alkylating agent which has both myelotoxic and immunosuppressive properties(1). Recently a regimen of moderate dose treosulfan has been used in combination with fludarabine and ATG for treatment of malignant lymphoma and a small number of patients with multiple myeloma with low transplant related mortality $(2,3)$. The aim with this retrospective single-center study was to evaluate recent transplants particularly after conditioning with treosulfan plus fludarabine (TreoFlu).

Material (or patients) and methods: The study comprised 39 patients (median age: 46 years) that underwent Allo-SCT between 2000 and 2015. Twenty-five patients were in very good partial response or better ( $\geq$ VGPR) and 14 in less than VGPR.

Results: The 3 years overall survival (OS) for the patients allografted after and before year 2008 was $89 \%$ vs $41 \%$ respectively $(\mathrm{HR}=0.14(95 \% \mathrm{Cl} 0.03-0.65, P=0.01)$ and for patients transplanted in $\geq$ VGPR and $P=0.18$ ). Eleven of the 22 patients transplanted after 2008 received TreoFlu. Only one of these patients died of NRM.

Conclusion: Thus, this single center study indicates improved survival following allogenic transplantation in multiple myeloma since 2008 perhaps in part due to encouraging use of TreoFlu for conditioning.

References: 1. Danylesko I, Shimoni A, Nagler A. Treosulfanbased conditioning before hematopoietic SCT: more than a BU look-alike. Bone Marrow Transplant. 2012 Jan;47(1):5-14.

2. Michallet M, Sobh M, Milpied N, Bay JO, Furst S, Harousseau $\mathrm{JL}$, et al. Phase II prospective study of treosulfan-based reduced-intensity conditioning in Allo-SCTgeneic HSCT for hematological malignancies from 10/10 HLA-identical unrelated donor. Annals of hematology. 2012 Aug;91(8):1289-97.

3. Schmidt-Hieber M, Blau IW, Trenschel R, Andreesen $R$, Stuhler $\mathrm{G}$, Einsele $\mathrm{H}$, et al. Reduced-toxicity conditioning with fludarabine and treosulfan prior to Allo-SCTgeneic stem cell transplantation in multiple myeloma. Bone Marrow Transplant. 2007 Apr;39(7):389-96.

Disclosure of Interest: None declared.

\section{AB63}

Feasibility of reduced toxicity treosulfan based conditioning for second allogeneic stem cell transplantation

J. Radocha ${ }^{1, *}$ A. Zavrelova ${ }^{1}$, M. Lanska ${ }^{1}$, B. Visek ${ }^{1}$, P. Zak ${ }^{1}$

${ }^{1}$ University Hospital Hradec Kralove, 4th Department of Medicine - Hematology, Hradec Kralove, Czech Republic

Introduction: Prognosis of relapse of hematologic malignancy after allogeneic stem cell transplantation remains dismal. Second allogeneic transplantation represents a valid clinical option in such patients. Toxicity of the preparative regimen for second transplanation may sometimes limit a decision making process in offering the patients a second graft. Goal was to evaluate the toxicity and tolerability of treosulfan, fludarabine and rabbit ATG (Thymoglobuline, r-TG) preparative regimen in the setting of the second allogeneic stem cell transplantation for disease relapse.

Material (or patients) and methods: We evaluated all patients who underwent second allogeneic transplantation for the relapse of their malignant disorder after the first allograft during years 2012-2015 at our department. Preparative regimen in all patients consisted of treosulfan $12 \mathrm{~g} / \mathrm{m} 2$ for 3 days, fludarabine $30 \mathrm{mg} / \mathrm{m} 2$ for 5 days and $\mathrm{r}-\mathrm{TG} 6 \mathrm{mg} / \mathrm{kg}$. All patients received calcineurin inhibitor and mycophenolate mofetil as GVHD prophylaxis. Day +28 and +100 mortality, platelet and neutrophil engraftment as well as infectious and acute non-infectious complications were recorded.

Results: We identified 9 patients ( 8 males and 1 female), median age 47 years (33-65 years) who underwent second allogeneic graft for relapsed disease after the 1st transplantation. 5 patients had acute myeloid leukemia or myelodysplastic syndrome, 2 patients had acute lymphoblastic leukemia, 1 had chronic lymphocytic leukemia and 1 primary myelofibrosis. 3 patient were in subsequent CR of the disease and 6 in relapse. 7 patients received 10/10 matched unrelated peripheral blood stem cell graft and 2 patients received $9 / 10$ mismatched unrelated peripheral blood stem cell graft. All patients engrafted in platelets at median D+13 (9-26) and in neutrophils at median D+14 (11-20). Median D+28 donor chimerism was $100 \%$ (5-100\%), one patient experienced graft failure at D+28 due to HHV-6 and subsequently died of HHV-6 encephalitis (confirmed). No other early deaths until D+100 were observed. Only 2 patients had mucositis, only one being grade 4. All 9 patients experienced episode of fever, 5 of them diagnosed with G- bloodstream infection and 2 of them with $G$ + blodstream infection. 2 patients had pneumonia. All patients recovered from their respective complications. Overall D+100 non-relapse mortality was $11.1 \%$ (1/9 patients).

Conclusion: Treosulfan, fludarabine and $\mathrm{r}$-TG is feasible option in the conditioning of relapsed patients undergoing the second allograft. Sunstained engraftment and acceptable early non-relapse mortality warrant its use in the setting of second transplant. Infection remains the biggest challenge in this setting.

Disclosure of Interest: None declared.

\section{AB64}

Splenic irradiation immediately before allogeneic stem cell transplantation in advanced phase myelofibrosis with massive splenomegaly

M. Parma ${ }^{1, *}$, C. Julita ${ }^{2}$, E. Elli ${ }^{1}$, R. Renso ${ }^{1}$, E. Terruzzi ${ }^{1}$, M. Fedele ${ }^{1}$, P. Pioltelli ${ }^{1}$

${ }^{1}$ Hematology, ${ }^{2}$ Radiotherapy, Ospedale San Gerardo, Monza, Italy

Introduction: Allogeneic hematopoietic stem cell transplantation (HSCT) in advanced phase myelofibrosis (MF) is a valid 
curative approach, but it is charged of an high risk of graft failure or rejection. This complications is though to be related to the particular condition of marrow micro-environment, but also spleen enlargement can play a role either in sequestering donor stem cells or in preserving autologous foci of stem cells. A proportion of MF patients (pts) can benefit from splenectomy before HSCT but such procedures have been associated with substantial surgical morbility.

Considering these, we have tried an alternative approach with splenic irradiation immediately before HSCT in a limited number of MF pts, and we evaluated the post transplant outcome.

Material (or patients) and methods: We selected 5 pts with advanced chronic phase MF (according to Cervantes scoring system 2 pts intermediate- 2 and 3 pts high risk IPSS) and one patient with acute evolution of MF; only one have been treated before with Ruxolitinib, all of them have a great spleen enlargement ranging from 18 to $25 \mathrm{~cm}$ (ultrasonography measured). All pts received a schedule of fractioned splenic irradiation as a component of conditioning regimen (4 Gy divided in 4 daily doses each of $1 \mathrm{~Gy}$, between - 15 to -5 days to transplant).

Conditioning regimen was Busulfan-Fludarabine for 4 pts, reduced dose Busulfan-Fludarabine for 1 patient and ThiotepaBusulfan-Fludarabine for 1 patient.

Results: after fractioned splenic irradiation and fludarabinebased conditioning regimen, all pts showed remarcable spleen volume reduction within two months after HSCT; $5 / 6$ pts obtained successful donor cells engraftment, without severe complication related to splenic irradiation. Median neuthrophil and platelet recovery $\left(>500 / \mathrm{mm}^{3}\right.$ and $\left.>20.000 / \mathrm{mm}^{3}\right)$ was 27 and 36 days respectively. After 2 months, chimerism status was $100 \%$ donor for 4 pts and $80 \%$ donor for 1 patient. Three pts had a good outcome and are actually disease free with a complete chimeric status after 39, 27 and 12 months from HSCT.

One patient relapsed and rapidly presented a fatal leukaemic evolution two months after HSCT, one patient relapsed with autologous haemopoiesis 6 months after HSCT. The only patient who did not engraft remained cytopenic surviving 12 months.

Conclusion: Splenic irradiation immediately before HSCT could be considered a safe and alternative option to splenectomy for MF pts with massive splenomegaly, in order to obtain the improvement of donor engraftment. Our limited experience shows that also these pts can have a favourable outcome. A largest number of cases would be required to discriminated the real advantage of this strategy.

Disclosure of Interest: None declared.

AB65

Results of observational study on treatment patterns in adult patients with hematological malignancy (HM) undergoing hematopoietic stem cell transplantation (HSCT) in North Africa, Turkey, Middle and Near East, and Eurasia

N. Mikhailova ${ }^{1, *}$, R. Ahmed Nacer ${ }^{2}$, R. Abdelfattah ${ }^{3}$, M. Vaezi $i^{4}$ H. Kamel ${ }^{5}$, G. Saydam ${ }^{6}$, T. Ben Othman ${ }^{7}$, A. Ibrahim ${ }^{8}$, B. Meddeb ${ }^{9}$, M. Ramzio, M. Moussa ${ }^{11}$, T. Zagoskina ${ }^{12}$, M.-L. Risse ${ }^{13}$, A. Zaristkiy ${ }^{14}$

${ }^{7}$ First St. Petersburg Pavlov State Medical University, St. Petersburg, Russian Federation, ${ }^{2}$ Centre Pierre et Marie Curie, Alger, Algeria, ${ }^{3}$ Sheikh Zayed Hospital, Cairo, Egypt, ${ }^{4} \mathrm{HORCSCT}$, Shariati Hospital, Tehran, Iran, Islamic Republic Of, ${ }^{5}$ Nasser Institute, Cairo, Egypt, ${ }^{6}$ Ege Universitesi Tip Fakültesi Hematoloji BD, Izmor, Turkey, ${ }^{7}$ National Bone Marrow Transplant Center, Tunis, Tunisia, ${ }^{8}$ Makassed General Hospital Tarik el Jdideh, Beirut, Lebanon, ${ }^{9}$ Hematology Department Hospital Aziza Othmana, Tunis, Tunisia, ${ }^{10}$ Namazi Hospital, Shiraz, Iran, Islamic Republic Of, ${ }^{11}$ Ain Shams University Hospital, Cairo, Egypt, ${ }^{12}$ Research Institute of blood transfusion, Kirov, Russian Federation, ${ }^{13}$ Sanofi Aventis Groupe, Gentilly, France, ${ }^{14}$ Federal Center of Heart, Blood \& Endocrinology, St. Petersburg, Russian Federation
Introduction: Available information on HSCT comes mainly from US and European countries. The purpose of this multinational prospective observational study was to collect data on treatment patterns of HSCT in HM in some non-US and non-EU countries.

Material (or patients) and methods: 500 patients (pts) were planned. Main eligibility criteria: adult pts with HM starting conditioning treatment in purpose of allogenic (allo) or autologous (auto) HSCT, written informed consent. Primary endpoint: to describe type of conditioning used in HSCT. Visit at 3 and 6 months after HSCT were planned.

Results: 500 eligible pts were recruited over 13 months in 9 countries, median age was 44 years old (min-max: 18-72), PS was 0 in $53.0 \%$ pts and 1 in $40.4 \%$ pts. HM were multiple myeloma (MM) (37.2\%), acute myeloid leukemia (19.8\%), nonHodgkin lymphoma (14.4\%), Hodgkin lymphoma (HD) (13.6\%), and acute lymphoblastic leukemia (9.2\%). Myeloablative (MA) conditioning regimen was most frequently used in all tumor types (overall $78.6 \%$ pts) and in allo and auto HSCT $(80.3 \%$ and $76.5 \%$, respectively). Source of HSC was peripheral blood in 91.4\% HSCT. Median day of engraftment was 13. Graft failure was reported in 18 pts (10.1\%) with allo HSCT and 2 pts (0.6\%) with auto HSCT.

In 309 pts auto HSCT from peripheral blood only, mobilization was chemotherapy (CT)+G-CSF in $40.5 \%$ pts, CT alone in $32.0 \%$ pts and G/GMCSF alone in $25.2 \%$ pts. CXCR4 antagonist was used in $2.3 \%$ pts. Median number of CD34+ cells prior first apharesis was $47 / \mu \mathrm{L}$ (min-max: 0.7-11748) and median number of apharesis was 2 (min-max: 1-8).

In 179 pts allo HSCT, $43.6 \%$ had ABO mismatch. GvHD prophylaxis was part of conditioning regimen in $26.8 \%$ pts and was mainly rabbit ATG (93.0\%). Acute GvHD any grade was reported in $38.0 \%$ pts and grade $3-4$ in $12.3 \%$ pts, and chronic GvHD any grade in $16.8 \%$ pts and grade $3-4$ in $1.7 \%$ pts. Majority of HSCT were CMV D+/R+ (73.2\%). CMV prophylaxis at 3 months was given in $29.1 \%$ pts and median duration was 90 days (min-max: $9-180$ ). CMV reactivation was reported in 31 pts and CMV infection in 19 pts.

Febrile neutropenia, diarrhea and neutropenic bacterial infection were the most frequent events $(47.6 \%, 24.6 \%$, and $14.8 \%$, respectively).

Conclusion: MA was the most frequent conditioning regimen used in both allo and auto HSCT with similar complications to western countries, and majority had auto HSCT.

Disclosure of Interest: N. Mikhailova Funding from: Sanofi, R. Ahmed Nacer Funding from: Sanofi, R. Abdelfattah Funding from: Sanofi, M. Vaezi Funding from: Sanofi, H. Kamel Funding from: Sanofi, G. Saydam Funding from: Sanofi, T. Ben Othman Funding from: Sanofi, A. Ibrahim Funding from: Sanofi, B. Meddeb Funding from: Sanofi, M. Ramzi Funding from: Sanofi, M. Moussa Funding from: Sanofi, T. Zagoskina Funding from: Sanofi, M.-L. Risse Employee of: Sanofi, A. Zaristkiy Funding from: Sanofi.

\section{AB66}

Immunologic recovery after rabbit Anti-ThimocyteGlobuline (ATG) in patients submitted to Matched Unrelated Donor (MUD) Hematopoietic Stem Cell Transplantation and its correlation with opportunistic infection during the following year

M. Parma ${ }^{1, *}$, R. Renso ${ }^{1}$, E. Terruzzi ${ }^{1}$, M. Fedele ${ }^{1}$, P. Pioltelli ${ }^{1}$

${ }^{1}$ Hematology, Ospedale San Gerardo, Monza, Italy

Introduction: In Europe rabbit Anti-Thimocyte-Globuline (ATG) is widely employed in patients submitted to Matched Unrelated Donor (MUD) Hematopoietic Stem Cell Transplantation (HSCT) in order to reduce acute and chronic Graft versus Host Disease (GvHD). ATG seems to be very effective in reducing GvHD, but a significant increase of infective complications is often observed in these patients (pts). This fact and the reduction of "Graft versus Leukemia" effect make the use of ATG in MUD-HSCT a matter of debate nowadays. We evaluated the immunologic recovery in pts treated with ATG during the first year after MUD-HSCT and its correlation with the 
insurgence of opportunistic infection in the same period, excluding infection appeared before hematological uptake.

Material (or patients) and methods: We considered 32 pts, affected by hematological malignancies, submitted to MUDHSCT who received ATG $7.5 \mathrm{mg} / \mathrm{Kg}$ during the conditioning regimen. We discriminated two categories: pts who developed opportunistic infection (12 pts) and pts without infection (20 pts) in the first year after HSCT. We analysed immunological recovery (lymphocyte sub population count) in these two series at two, four, six eight and twelve months after HSCT.

Results: The more frequent infection is Cytomegalovirus reactivation (8 cases), the others are Pneumocystis Carinii Pneumonia (2 cases), EBV reactivation, other Pneumonia, Varicella-Zoster reactivation.

The majority (9/12) of pts who developed infections has been treated previously with ATG. On the contrary, only a minority of pts $(6 / 20)$ who didn't develop infections received ATG.

Analysing data about immunological reconstitution, we have seen that pts who develop infections have a lower count of $T$ cells and particularly of T4, not only immediately after HSCT but also during the entire observation time. B and NK cells and T4/T8 ratio are lower in these pts for all the period, although the statistical significance is not reached due to the limited number of pts. Conclusion: Pts who receive ATG have a higher susceptibility to infections respect to pts who do not. Pts with infections have a slower immunological recovery than expected.

We can conclude that pts who receive ATG during the conditioning regimen should be strictly monitored for immunological recovery and for infection insurgence. The real role of ATG in this prolonged immunological recovery remain to be defined. Disclosure of Interest: None declared.

\section{AB67}

Factors affecting overall survival in children following hematopoietic stem cell transplantation: a single institution analysis

A. Zaucha-Prażmo ${ }^{1, *}$, K. Drabko ${ }^{1}$, M. Choma ${ }^{1}$, B. Wójcik², J. R. Kowalczyk

${ }^{1}$ Department of Pediatric Hematology, Oncology and Transplantology, Medical University of Lublin, ${ }^{2}$ Department of Pediatric Hematology, Oncology and Transplantology, Children University Hospital, Lublin, Poland

Introduction: Successful outcome of hematopoietic stem cell transplantation (HSCT) is limited by serious procedure related complications.

Material (or patients) and methods: We retrospectively analyzed the treatment outcome in 185 children ( 96 boys and 89 girls), with median age 8,7 years (range 0,1-18,5 years) treated with HSCT from June 2005 to October 2015 in one institution. The underlying diseases were malignant in 139 pts (75\%): ALL -38, AML- 34, lymphomas -19, MDS - 11, CML-1, solid tumors -36 and non-malignant in 46 pts (25\%): SAA - 13, PID -19, congenital anemia-7, congenital metabolic disorders- 7. According to the type of transplant: 47 (25\%) were autologous and 138 (75\%) allogeneic, of which 48 from MSD (34,8\%); 78 from MUD (56,5\%) and 12 from MMFD (8,7\%). Stem cell sources included bone marrow $(n=97)$, peripheral blood $(n=$ $87)$ and cord blood $(n=1)$. PBSC were the source of transplant in 45/47 autologous recipients, $2 / 47$ received BM. The majority of allo transplant recipients (95/138) received BM, 42/138 PBSC and $1 / 138$ CB. GvHD grade $=>2$ was diagnosed in $40 / 138$ allotransplant recipients (29\%). Statistical analysis of OS was performed using Kaplan-Mayer method and long rank and chi square tests. Statistical significance was considered $<0,05$.

Results: With a median follow-up of 3-years overall survival (OS) was 0,71 in the whole group of 185 patients. According to the type of transplant OS in MSD recipients was 0,82; in MUD and auto 0,65 ; haplo $0,47(P=0,01)$. In allo transplants OS was significantly higher in $\mathrm{BM}$ recipients comparing to PBSC recipients $(0,76$ versus $0,5 P=0,002) .53 / 185$ pts $(29 \%)$ died, $31 / 185$ pts of disease relapse or progression ( 15 allo versus 16 auto recipients), 22/185 pts died of transplant related complications, all in alloHSCT group - 7 pts in non-malignant and 15 pts in malignant group [table 1].

Conclusion: Post-transplant relapse remained the main cause of death in analyzed group of patients. Main causes of transplant related mortality were organ toxicities and infection, not GvHD. Factors significantly affecting overall survival were donor type and stem cell source.

Disclosure of Interest: None declared.

\section{AB68}

Late Effects of Allo-HSCT in Children: The Experience of a Single Pediatric Transplant Center. Comparison of Malignant and Non-Malignant Diseases

A. Gefen ${ }^{1, *}$, M. Peretz-Nahum ${ }^{1}$, M. Weyl Ben-Arush ${ }^{1}$, I. Porat ${ }^{1}$, A. Khalil', I. Zaidman

${ }^{7}$ Pediatric Hematology Oncology Division, Rambam Medical Center, Haifa, Israel

Introduction: As the survival of allo-HSCTs is constantly improving, more and more patients, especially the youngest, are susceptible to late complications. Here, we present our institutional experience of late effects in the first 6 years $(y)$ following pediatric allo-HSCT, comparing malignant versus non-malignant indications for HSCT.

Material (or patients) and methods: All children allotransplanted between 2009 and 2015 were included in this single- center retrospective cohort. Screening was carried out in accordance with international published guidelines. ${ }^{1}$ Of 76 transplanted patients, 58 were included in the analysis; 18 excluded due to short ( $<6$ months) follow-up.

Results: Median age at HSCT was 9.8y; the non-malignant group was younger. HSCT indications were evenly divided between non-malignant (NMD) and malignant diseases (MD) ( $82 \%$ of which were $A L L)$. Ten patients $(17 \%)$ required a $2^{\text {nd }}$ HSCT. Pre-transplant characteristics are listed in table 1. Median follow-up was 3.3y. Twenty one patients (36\%) had CGVHD; the rate of CGVHD was double in patients with MD, and all 5 severe cGVHD cases occurred in this group. The common affected organs were skin \& joints, Gl tract, lungs,

[AB67]

Table 1: Transplant related deaths in alloHSCT recipients.

\begin{tabular}{lccccccccc}
\hline TRM in alloHSCT $(n)$ & $\begin{array}{c}\text { MOF } \\
(n)\end{array}$ & $\begin{array}{c}\text { CNS toxicity } \\
(n)\end{array}$ & $\begin{array}{c}\text { Pulmonary } \\
\text { toxicity }(n)\end{array}$ & $\begin{array}{c}\text { IFI } \\
(n)\end{array}$ & $\begin{array}{c}\text { Bacterial infection } \\
(n)\end{array}$ & $\begin{array}{c}\text { PTLD } \\
(n)\end{array}$ & $\begin{array}{c}\text { Bleeding } \\
(n)\end{array}$ & $\begin{array}{c}\text { GvHD } \\
(n)\end{array}$ & $\begin{array}{c}\text { CMV infection } \\
(n)\end{array}$ \\
\hline $\begin{array}{l}\text { Malignant }(n=15) \\
\begin{array}{l}\text { Non-malignant } \\
(n=15)\end{array}\end{array}$ & 3 & 2 & 2 & 2 & 1 & 1 & 2 & 1 & 1 \\
All $(n=22)$ & 6 & 2 & 1 & 1 & 1 & 1 & 0 & 0 & 0 \\
\end{tabular}

TRM transplant related mortality; MOF multiorgan failure; IFI invasive fungal infection. 
Table 1: patients' characteristics and outcomes

\begin{tabular}{|c|c|c|c|}
\hline & All children & Malignant diseases & Non-malignant diseases \\
\hline Number of patients (\%) & $58(100 \%)$ & $28(48 \%)$ & $30(52 \%)$ \\
\hline Males (\%) & $31(53 \%)$ & $18(64 \%)$ & $13(43 \%)$ \\
\hline Median age on HSCT (range) & $9.8 y(0.3-19)$ & $12.6 y(0.6-18)$ & 4.3y (0.3-19) \\
\hline Median follow-up (range) & $3.3 y(0.7-6)$ & $2.8 y(0.8-5.4)$ & $3.9 y(0.7-6)$ \\
\hline $2^{\text {nd }} \mathrm{HSCT}(\%)$ & $10(17 \%)$ & $5(18 \%)$ & $5(17 \%)$ \\
\hline cGVHD - total (\%) & $21(36 \%)$ & $14(50 \%)$ & $8(26 \%)$ \\
\hline cGVHD - severe (\%) & $5(9 \%)$ & $5(18 \%)$ & $0(0 \%)$ \\
\hline Late effects* $(\%)$ & $21(36 \%)$ & $13(46 \%)$ & $8(26 \%)$ \\
\hline \multirow{3}{*}{ Mortality (\%) } & $8(14 \%)$ & Total: 7 (25\%) & $1(3 \%)$ \\
\hline & & Relapse: 6 (21\%) & Relapse: 1 (3\%) \\
\hline & & HSCT complications: 1 (3\%) & HSCT complications: 0 (0\%) \\
\hline
\end{tabular}

* Excl. Vitamin D deficiency.

eyes \& liver. Late complications included hormonal dysfunction in 13 patients $(22 \%) ; 80 \%$ of gonadal insufficiency cases were in females. More than half (57\%) had vitamin D deficiency. Other involved systems included: cardiac (9\%), pulmonary (7\%), and skeletal (osteoporosis \& avascular necrosis - 7\%). The risk of late complications was not increased following $2^{\text {nd }} \mathrm{HSCT}$ (30 vs. $35 \%$ ), although cGVHD incidence was higher ( 50 vs. $36 \%$ ). Seven patients with MD died during the follow-up period compared to 1 case in the NMD group. See table 1 for transplant related morbidity and mortality.

Conclusion: These data confirm that even in the first years following allo-HSCTs, pediatric patients are prone to a diverse array of complications, mainly endocrine. The underlying disease appears to affect morbidity: children with MD have a two-fold risk of developing CGVHD and late effects (other than vitamin $\mathrm{D}$ deficiency), versus NMD. These differences could be attributed to higher cumulative induction/conditioning radio-chemotherapy dose in the MD group. Although the vast majority of deaths were in patients with $M D$, most deaths were attributed to disease relapse; therefore no conclusion can be drawn regarding late transplant related mortality in MD versus NMD. Study limitations include the relatively small cohort, loss to follow-up and uncertainty regarding patient adherence to screening guidelines. As long-life follow-up is needed for this unique population, a continuing surveillance of the cohort study is planned.

References: 1. Bone Marrow Transplant. 2012;47:337-41.

Disclosure of Interest: None declared.

\section{AB69}

Positive mrd after haploidentical stem cell transplant for all: donor lymphocites infusion and GVHD, a challenging combination

B. Torres Guerola ${ }^{1, *}$, C. Fuentes Socorro ${ }^{7}$, M. D. M. Andrés Moreno ${ }^{1}$, I. Gómez Seguí ${ }^{2}$ J. M. Fernández Navarro ${ }^{1}$

${ }^{1}$ Paediatric Bone Marrow Transplant, ${ }^{2}$ Hematology and chemotherapy, Hospital La Fe, Valencia, Spain

Introduction: Donor lymphocytes infusion (DLI) is a recognised practice for the treatment of positive minimal residual disease (MRD) after stem cell transplant. Its use is not indicated in the presence of GVHD. In the cases of GVHD post DLI, the beneficial effect could be impaired by the use of steroids.

Material (or patients) and methods: We report our experience in the management of a child with acute skin GVHD and positive MRD detected after haploidentical stem cell transplant (haplo SCT) for ALL.

Results: Two years old female, diagnosed of precursor B acute lymphoblastic leukemia. Treated as per the national protocol. Indication for allogenic stem cell transplant for positive MRD after consolidation chemotherapy. Because of the absense of matched related or unrelated donor, father was selected as haploidentical donor (for NK alloreactivity). Prior to transplant she was in complete remission (CR) with negative MRD. Conditioning regime: thiotepa, busulfan and fludarabine. GVHD prophylaxis: Post- infusion ciclophosphamide on days $+3,+4$ and tacrolimus + MMF from day +5 . Unmanipulated peripheral blood was infused $\left(\mathrm{CD} 34+6 \times 10^{6} / \mathrm{kg}\right)$. Neutrophil engraftment on day +16 . On day +23 she was diagnosed of acute gut and skin GVHD (grade II). She was started on systemic steroids (methylprednisolone $2 \mathrm{mg} / \mathrm{kg} /$ day) and presented very good response by day 7 , so steroids were tapered. Bone marrow aspirate (BMA) on day +30 : full donor chimerism, negative MRD. MMF discontinued on day +30 as per protocol. BMA on day +60 showed morphological CR, positive MRD $(0.11 \%)$ and $96 \%$ donor chimerism. Tacrolimus and prednisone were suspended. She received a DLI on day +70 (CD3+ $1 \times 10^{5} / \mathrm{kg}$ ). Prior to DLI she presented malar exanthema with no other signs of GVHD. One week after the DLI she presented with generalised macular exanthema including palms and plants compatible with skin GVHD. No other organs affected. She was started on topical steroids and extracorporeal photopheresis (ECP, 2 sessions per week) to avoid the use of systemic steroids. BMA on day+80: MRD $0.02 \%$. In the context of GVHD no more DLI were administered. MRD on day $+90: 0.007 \%$. GVHD progressively improved with ECP treatment. She is now a year post- transplant. MRD remains negative and skin GVHD is resolved. ECP has now been stopped after 23 sessions.

Conclusion: DLI is a useful tool in the management of positive MRD early after transplant in paediatric ALL. ECP permitted the avoidance of steroids in GVHD recurrence after DLI.

References: HJ Kolb et al. Graft-versus-leukemia effect of donor lymphocyte transfusions in marrow grafted patients. European Group for Blood and Marrow Transplantation Working Party Chronic Leukemia. Blood Sep 1995; 86 (5): 2041-2050.

Disclosure of Interest: None declared.

\section{AB70}

Hematopoietic stem cell transplantation for primary immunodeficiencies. The experience of the Transplant Center Timisoara

C. Jinca ${ }^{1,2, *},{ }_{3}$ M. Serban ${ }^{2}$, M. Bataneant ${ }^{1}$, A. Oprisoni ${ }^{1}$, A. Isac ${ }^{2}$, A. Pascalau', A. Balan', M. Baica ${ }^{4}$, S. Arghirescu'

${ }^{1}$ Pediatrics, University of Medicine "V. Babes", ${ }^{2}$ Bone Marrow Transplantation, ${ }^{3}$ Onco-hematology, ${ }^{4}$ Flowcytometry lab, Emergency children's Hospital "L. Turcanu", Timisoara, Romania

Introduction: Allogeneic hematopoietic stem cell transplantation (HSCT) represents an established indication for several lethal primary immunodeficiencies (PIDs). 
Material (or patients) and methods: We aim to present the outcome of six pediatric patients with PID transplanted at the Bone Marrow Transplant Center in Timisoara/ Romania. Three children with $x$-linked chronic granulomatous disease (CGD), one patient with Griscelli syndrome type 2 and hemophagocytic lymphohistiocytosis (HLH) and two other twins with IL-7 receptor deficient $\mathrm{T}^{+} \mathrm{B}^{+} \mathrm{NK}^{+}$severe combined immunodeficiency (SCID) were transplanted from matched related or unrelated donors at our Center between 2008-2014. All three patients with CGD received myeloablative regimens and the grafts were bone marrow in two and peripheral blood stem cells (PBSC) in one of the patients. The patient with Griscelli received also a myeloablative regimen and PBSC.The two SCID's had a non-myeloablative conditioning and were transplanted with bone marrow simultaneously.

Results: All of the patients engrafted, presenting complete donor chimerism early after the transplant. Regarding transplant specific complications, two patients presented acute graft versus host disease and the child with HLH developed severe veno-occlusive disease early after the transplant. Two out of the three patients with CGD are cured, the third died following severe pneumonia at 3 months posttransplant with $100 \%$ donor chimerism and normal function of granulocytes and monocytes. One of the twins with SCID is well with $100 \%$ donor chimerism and good immune reconstitution, the other one died of pulmonary complications, whereas the patient with $\mathrm{HLH}$ is also in remission, with a mixed chimerism. Overall, four of six patients are alive and well with complete recovery of their severe immune deficiency.

Conclusion: HSCT provided cure for two thirds of the patients with PID. Although HLA-matched sibling donors are the best option for these patients, the advances in HLA typing and unrelated donor search have made HLA-matched unrelated donor transplants a very good alternative.

Disclosure of Interest: None declared.

\section{AB71}

Stem Cell Transplantation for Non-Malignant diseases in a Tertiary Center in Singapore: A 10-Year Review

F. Yeap ${ }^{1,2, *}$, K. Francisco ${ }^{1}$, M. Villegas ${ }^{1}$, P.-L. Tan ${ }^{1,2}$

${ }^{1}$ Pediatrics, Khoo Teck Puat- National University Children's Medical Institute, ${ }^{2}$ Pediatrics, Khoo Teck Puat- National University Children's Medical Institute, National University Hospital, Singapore, Singapore, Singapore

Introduction: We retrospectively reviewed the outcome of 33 hematopoietic stem cell transplantation in 25 patients with non-malignant diseases treated at National University Hospital, Singapore, over the period of 10 years, from June 2005 to August 2015.

Material (or patients) and methods: The age of the patients ranged from 7 months to 17.5 years with a median of 4.76 years. There were 16 male and 9 female patients. The conditions managed were namely that of Thalassaemia $(n=8)$, Aplastic Anaemia $(n=4)$, Haemophagocytic lymphohistiocytosis/ Lymphoproliferative Disorder $(n=6)$, Immunodeficiencies (Chronic Granulomatous Disease (CGD), Severe Combined Immunodeficiencies (SCID), CD40 Ligand deficiency) $(n=4)$ and others (Congenital Haemolytic Anaemia, Diamond Blackfan Anaemia, Dyskeratosis Congenita) $(n=3)$.

Results: There were 6 matched sibling donor (MSD) transplants, 2 matched related donor (MRD) transplants, 17 matched unrelated donor (MUD) transplants and 8 haploidentical transplants. Stem cell sources were bone marrow $(n=15)$ peripheral blood stem cell $(n=15)$ and dual umbilical cord blood $(n=2)$. TNC count ranged from $1.74 \times 10^{8} / \mathrm{kg}$ to $129.73 \times 10^{8} / \mathrm{kg}$ with a mean of $23.1 \times 10^{8} / \mathrm{kg}$ and CD34+ count ranged from 0.13 $\times 10^{6} / \mathrm{kg}$ to $57 \times 106 / \mathrm{kg}$ with a mean of $12.8 \times 10^{6} / \mathrm{kg}$. Most of the conditioning regimens were myeloablative, but 8 out of the 32 transplants were reduced intensity. Four patients received TBI of 2 Gy each. At the median follow-up of 8.6 years (range 2.2-15.0 years) as of December 2015, 21 out of 25 patients are alive without disease, with 1 patient alive but living with disease.
Eleven patients developed acute GVHD (grade 1-2) and 3 of them went on to develop chronic GVHD; all successfully managed with steroids and a combination of tacrolimus, cyclosporine and mycophenolate. Interesting to note was that 5 out of the 6 patients with $\mathrm{HLH} / \mathrm{LPD}$ developed engraftment syndrome/ cytokine release syndrome. Of note there was a high incidence of graft failures with 7 primary and 6 secondary graft failures.

Conclusion: In the absence of a matched sibling and matched unrelated donor, haploidentical parental stem cells are more readily utilized especially when the disease progression is rapid and a transplant is urgent. With improved techniques in stem cell manipulation haploidentical parental tranplatation offers a cure for these patients. However more work needs to be done in evaluating the engraftment and immune reconstituition in these patients.

References:

1. CC Dvorak et al. Hematopoietic stem cell transplantation for primary immunodeficiency disease. Bone Marrow Transplantation (2008)41, 119-126.

2. V Rocha et al. Searching for alternative stem cell donors for pediatric patients. Bone Marrow Transplantation (2008)41, 207-214.

Disclosure of Interest: None declared.

\section{AB72}

Hematopoietic Stem Cell Transplantation for Pediatric Mixed Phenotype Acute Leukemia

J. A. Park ${ }^{1, *}$, J. M. Lee ${ }^{2}$, K. S. Lee ${ }^{3}$, J. Y. Kim ${ }^{3}$, J. Y. Lim ${ }^{4}$, E. S. Park ${ }^{4}$, H. S. Kim ${ }^{5}$, Y. J. Shim ${ }^{5}$, E. J. Choi ${ }^{6}$, H. Chueh ${ }^{7}$, J. Park ${ }^{8}$, S. K. Park ${ }^{9}$, J. O. $\mathrm{Hah}^{2}, \mathrm{~J} . \mathrm{SeO}^{10}$, Y. T. $\mathrm{Lim}^{10}$ on behalf of on the behalf of Youngnam Pediatric Hematology and Oncology Society

${ }^{1}$ Pediatrics, Inje University Haeundae-paik Hospital, Busan, ${ }^{2}$ Pediatrics, Yeungnam University Hospital, ${ }^{3}$ Pediatrics, Kyungpook National University Hospital, Daegu, ${ }_{5}^{4}$ Pediatrics, Gyeongsang National University Hospital, Jinju, ${ }^{5}$ Pediatrics, Keimyung University Dongsin Medical Center, ${ }^{6}$ Pediatrics, Daegu Catholic University Hospital, Daegu, ${ }^{7}$ Pediatrics, Dong-A University Hospital, ${ }^{8}$ Pediatrics, Inje University Busan-Paik Hospital, Busan, ${ }^{9}$ Pediatrics, Ulsan University Hospital, Ulsan, ${ }^{10}$ Pediatrics, Busan National University Hospital, Busan, Korea, Republic Of

Introduction: Mixed phenotype acute leukemia (MPAL) is a rare disease accounting for about 2-5\% of all acute leukemias, and patients with MPAL have inferior outcome when compared with acute myeloid leukemia (AML) or lymphoid leukemia patients. However, there is no standardized treatment for MPAL and no consensus about the incorporation of hematopoietic stem cell transplantation (HCT) as an integral part of therapy. Due to its rarity, safety and efficacy of HCT for pediatric MPAL have not been fully determined, thus, we investigated the outcome of HCT for pediatric MPAL by multicenter study.

Material (or patients) and methods: We retrospectively analyzed transplant outcomes for 12 pediatric patients with primary MPAL patients, median age 9.0 years (range, 1.3-13.3 years), undergoing HCT between 2001 and 2014. By WHO 2008 classification, 1 (8\%) had MPAL with $\mathrm{t}(\mathrm{v} ; 11 \mathrm{q} 23)$; $M L L$ rearranged, 9 (75\%) had MPAL, T/myeloid, NOS, and the remaining $2(17 \%)$ had MPAL, B/myeloid, NOS. All patients were treated with induction chemotherapy targeted to AML and attained remission, and median time to complete remission was 43 days (range, 28-89 days). Before HCT, two patients relapsed at 4.2 and 7.7 months after diagnosis, respectively. One received further chemotherapies and attained second remission, but the other one proceeded HCT without further treatment. Donor was matched-related in 6 , matched-unrelated in 2, umbilical cord blood (UCB) in 2, and autologous in 2 , respectively. Conditioning regimen was busulfan, cyclophosphamide and/or melphalan based myeloablative (MA) in 10 and busulfan and fludarabine based nonmyeloablative (NMA) in 1 patient. One case of autologous $\mathrm{HCT}$ received cyclophosphamide, etoposide, cytarabine and $\mathrm{BCNU}$ as a conditioning chemotherapy. 
Results: Cumulative incidences of neutrophil and platelet engraftment by day 28 were $100 \%$. After median follow-up of 6.2 years (range, 0.8-17.4), 8 of 12 patients survived. Cumulative incidence of treatment-related mortality (TRM) and relapse were $9 \%$ and $35.8 \%$. Event-free survival (EFS) and overall survival (OS) after HCT were $58.3 \%$ (95\% Cl, 44\%-73\%) and $66.7 \%(95 \% \mathrm{Cl}, 53 \%-80 \%)$, respectively. WHO classification did not significantly affected EFS after HCT for pediatric MPAL patients $(P=0.371)$. Also, time to remission (less than 45 days or not) and stem cell donors were not significantly associated with EFS after HCT.

Conclusion: Many studies have reported that patients with MPAL had poor prognosis, and patients with $\mathrm{T} /$ myeloid phenotype had a worse outcome than patients with B/ myeloid phenotype. We found that WHO classification 2008 for MPAL was not significantly associated with patients' outcome after HCT and that patients with MPAL, T/myeloid, NOS had more favorable outcome compared to historical data. Our data suggested that HCT after complete remission may improve the outcome of pediatric patients with MPAL, particularly T/myeloid phenotype. This study needs to be confirmed in larger, prospective studies including more pediatric MPAL patients.

Disclosure of Interest: None declared.

AB73

Mortality following allogeneic hematopoietic stem cell transplantation among pediatric cancer patients in argentina

S. Gomez 1,* A. Fynn ${ }^{1}$, W. Goldman ${ }^{1}$, R. Ojha ${ }^{2}$

${ }^{1}$ Hospital de Niños Sor Maria Ludovica, La Plata, Argentina,

${ }^{2}$ St. Jude Children's Research Hospital, Memphis, United States

Introduction: Non-relapse mortality following allogeneic hematopoietic stem cell transplantation has rapidly decreased during the past 15 years among pediatric cancer patients in developed countries, but little information about non-relapse or relapse-related mortality is available for pediatric cancer patients in developing countries. We aimed to estimate the cumulative incidence of non-relapse and relapse-related mortality following allogeneic hematopoietic stem cell transplantation among pediatric cancer patients in Argentina.

Material (or patients) and methods: Individuals eligible for our study were aged $<20$ years when diagnosed with cancer and underwent allogeneic hematopoietic stem cell transplantation between September 1, 1998 and May 31, 2015 at Hospital de Ninos Sor Marlia Ludovica in La Plata, Argentina, which is a regional referral center for pediatric transplantation. Our outcomes of interest were non-relapse and relapse-related mortality, where non-relapse mortality was defined as mortality from any cause other than cancer progression. These outcomes were prospectively documented following transplantation until July 31 , 2015. We used a competing-risks framework to estimate cumulative incidence and $95 \%$ confidence limits $(C L)$ of nonrelapse mortality and relapse-related mortality.

Results: Our study population comprised 89 pediatric cancer patients, of whom $44 \%$ were aged $<10$ years at transplantation, $63 \%$ were male, $90 \%$ were diagnosed with leukemia, and $92 \%$ were transplanted with stem cells from related donors. We observed 39 deaths (24 relapse-related and 15 nonrelapse) during 280 person-years of follow-up (median $=3.6$ years, interquartile range $=1.2-8.4$ ). Infections or infectionrelated consequences were the most common causes of nonrelapse mortality (40\% of non-relapse deaths). The 5 -year cumulative incidence of non-relapse mortality was 18\% (95\% $\mathrm{CL}: 10 \%, 26 \%$ ) and the 5 -year cumulative incidence of relapserelated mortality was $31 \%$ (95\% CL: $21 \%, 42 \%)$. All non-relapse mortality in our population occurred within 14 months of transplantation, whereas the latest relapse-related mortality occurred nearly 10 years after transplantation.

Conclusion: Our results suggest that relapse-related mortality is the predominant reason for mortality 5 years post-allogeneic hematopoietic stem cell transplantation among pediatric cancer patients in Argentina. Nevertheless, non-relapse mortality is substantially higher in our population than posttransplant pediatric cancer patients in Europe (cumulative incidence $=9.0 \%{ }^{1}$ ), and the predominant reason for mortality within the first year following transplantation. Future research should aim to identify which modifiable factors can be targeted for intervention to help reduce non-relapse mortality following allogeneic hematopoietic stem cell transplantation in this population.

References: 1. Brissot E, Rialland F, Cahu X, et al. Improvement of overall survival after allogeneic hematopoieitic stem cell transplantation for children and adolescents: a three-decade experience of a single institution. Bone Marrow Transplant. 2015 Dec 7. doi: 10.1038/bmt.2015.250. [Epub ahead of print] Disclosure of Interest: None declared.

\section{AB74}

Individualized stem cell donor requests: Not longer a search in a haystack

G. Hütter,", A. J. Wensing ${ }^{2}$, J. D. Diez Martin ${ }^{3}$, J. Kuball ${ }^{2}$ M. Nijhuis', A. Saez-Cirion ${ }^{4}$, V. Rocha ${ }^{5}$, J. Schulze zur Wiesch ${ }^{6}$ J. Martinez-Picado ${ }^{7}$

${ }^{1}$ Cellex GmbH, Dresden, Germany, ${ }^{2}$ University Medical Center Utrecht, Utrecht, Netherlands, ${ }^{3}$ Hospital Gral. Univ. Gregorio Marañon, Madrid, Spain, ${ }^{4}$ Pasteur Institute, Paris, France, ${ }^{5}$ Oxford University Hospital, Oxford, United Kingdom, ${ }^{6}$ University Medical Center Hamburg-Eppendorf, Hamburg, Germany, ${ }^{7}$ AIDS Research Institute irsiCaixa, Barcelona, Spain

Introduction: The number of registered donors worldwide is increasing and frequently there is a choice between several HLA identical stem cell sources. To find the best donor, currently only limited information like gender, CMV etc. are available. The case of a successful stem cell transplantation (SCT) of an HIV infected patient ("Berlin Patient") with leukemia in 2007, where a donor with HIV resistance (homozygosity for CCR5-d32) was identified, emphasized the potential of individualized stem cell therapy.

Material (or patients) and methods: EpiStem (www.epistemproject.org) is a collaborative consortium to guide and investigate the potential for HIV cure in infected patients requiring allogeneic SCT for hematological disorders. Donor requests for 15 patients were collected between 2012 and today. Potential donors and cord blood sources were tested for the CCR5-d32 mutation. The DKMS Donor Navigator was used to pre-screen donors in the DKMS registry.

Results: The EpiStem Project has generated a prospective observational cohort of 15 cases of allogeneic SCT in HIV-1infected individuals with life-threatening hematological malignancies. Five cases had a CCR5-d32 donor and 10 had a CCR5 wild type donor. In 3 cases the donor cells came from a cord blood unit and in 12 cases from an adult donor. So far 3 patients have successfully passed the 6 months follow-up after transplantation, and 6 patients have died after transplantation, despite achieving full donor cell chimerism in some cases. Preliminary analysis of virological and immunological data from blood and tissue samples reveal a systematic reduction of HIV-1 reservoirs to very low levels. The DKMS Donor Navigator was successful in 5 of 11 requests even though not all patients entered into the EpiStem project (Table 1).

Conclusion: In the past additional genetically information from donors was not available or very hard to access. Requests for blood samples during CT/workup are possible but commonly limited to a few selected donors. In case of the "Berlin patient" the screening of 62 donor samples took several months of time which is generally too long for most of the patients. Prescreening of cord blood units was the first step in generating a preemptive data base of additional stem cell source information. The most recent development of the DKMS Donor Navigator allows transplant centers to prescreen a large number of donors already typed for additional and beneficial information (currently CCR5 and KIR1). In case of searches of CCR5-d32 homozygous donors for HIV infected 


\begin{tabular}{|l|l|c|c|c|c|c|}
\hline Patient & Side & $\begin{array}{l}\text { Episstem } \\
\text { patient }\end{array}$ & $\begin{array}{l}\text { DKMS } \\
\text { donors }\end{array}$ & $\begin{array}{l}\text { CCR5 pre- } \\
\text { tested }\end{array}$ & $\begin{array}{l}\text { CCR5-d32/d32 } \\
\text { donors }\end{array}$ & SCT \\
\hline 1 & Spain & Yes & $4^{*}$ & $100 \%$ & 0 & done \\
\hline 2 & Netherlands & Yes & 440 & $20 \%$ & 3 & done \\
\hline 3 & Netherlands & No & $8^{*}$ & 0 & - & stopped \\
\hline 4 & Belgium & Yes & $1\left(9^{*}\right)$ & 0 & - & pending \\
\hline 5 & USA & No & 2 & 0 & - & stopped \\
\hline 6 & Germany & Yes & $>4000$ & $45 \%$ & 7 & pending \\
\hline 7 & Germany & Yes & $4^{*}$ & 0 & - & pending \\
\hline 8 & Germany & Yes & $>100$ & $>30 \%$ & 1 & pending \\
\hline 9 & Netherlands & Yes & $>1000$ & $>30 \%$ & 8 & pending \\
\hline 10 & Canada & No & $>1000$ & $>30 \%$ & 17 & pending \\
\hline 11 & Germany & Yes & 20 & $10 \%$ & 0 & pending \\
\hline
\end{tabular}

Table 1

Results of the CCR5 genotype screening performed using the DKMS Donor Navigator " *HLA match $9 / 10)$

patients the DKMS Donor Navigator was successful in $45 \%$ of the cases. Identifying genetic determinants in transplantation, prescreening the donors, and sharing this information with the clinicians may deeply improve the quality of donor selection in the future.

Disclosure of Interest: None declared.

\section{AB75}

Infusion rates of the graft do not influence early engraftment following intra bone marrow transplantation in a DLA-identical canine transplantation model

S. Schäfer ${ }^{1,2, *}$, J. Werner ${ }^{1}$, K. Neumann ${ }^{1}$, C. Machka ${ }^{1}$, S. Lange ${ }^{1}$ G. Knuebel', A. Sekora ${ }^{1}$, C. Roolf ${ }^{1}, H$. Murua Escobar ${ }^{1,2}$, H. Vogel ${ }^{\prime}$, I. Lindner', S. Kamysek', I. Nolte', C. Junghan $\beta^{1}$

${ }^{1}$ Hematology/Oncology/Palliative Care, University of Rostock, Rostock, ${ }^{2}$ Small Animal Clinic, University of Veterinary Medicine Hannover, Hannover, ${ }^{3}$ Clinic for Radiotherapy, ${ }^{4}$ Institute of Legal Medicine, ${ }^{5}$ Anesthesiology and Intensive Care Medicine, University of Rostock, Rostock, Germany

Introduction: Intra bone marrow transplantation (IBMT) of hematopoietic stem cells (HSC) might be a promising alternative to conventional intravenous application of HSC in order to improve the homing efficiency and to accelerate the early engraftment after transplantation. Especially for transplantation of limited cell numbers the IBMT might prevent a delayed engraftment. Here we investigated the impact of the graft infusion rate on the hematopoietic recovery as well as on the engraftment kinetics after allogeneic IBMT in a canine DLA-identical littermate model following reduced intensity conditioning.

Material (or patients) and methods: Dogs were conditioned with $4.5 \mathrm{~Gy}$ total body irradiation. The graft volume was reduced by buffy coat centrifugation and dogs obtained $2 \times 25 \mathrm{ml}$ into the femur and humerus simultaneously. The infusion period of the graft was $10 \mathrm{~min}$ in group 1 (IBM10, $n=8$ ) and $60 \mathrm{~min}$ in group 2 (IBM60, $n=7$ ). Cyclosporin A (15 mg/kg, BID) was administered as immunosuppression from d-1 until $\mathrm{d}+35$ post transplantation. The hematopoietic recovery was monitored daily by blood counts and the development of the chimerism for the peripheral blood mononuclear cells (PBMC) and granulocytes was determined weekly.

Results: All animals of both groups engrafted. One dog died at $\mathrm{d}+15$ (infection) of the IBM10 group and was therefore not included. The median number of infused total nucleated cells was $4.1^{*} 10^{8} / \mathrm{kg}$ in both groups (IBM10: range $2.3-6.0^{*} 10^{8} / \mathrm{kg}$, IBM60: range 1.8-4.4*10 $10^{8} / \mathrm{kg} ; P=$ n.s.). The infused $\mathrm{CD} 34^{+}$cell numbers were median $3.2^{*} 10^{6} / \mathrm{kg}$ (range: $1.2-10.0^{*} 10^{6} / \mathrm{kg}$; IBM10) and $3.6^{*} 10^{6} / \mathrm{kg}$ (range: $1.5-6.8^{*} 10^{6} / \mathrm{kg}$; IBM60) $(P=\mathrm{n}$. s.). Time of leukocyte recovery was median $d+11$ (range: $9-16)$ and $\mathrm{d}+10$ (range: $8-14)$ after 10 and 60 min infusion $(P=$ n.s.).
Median leukocytes nadirs amounted to $0.24^{*} 10^{9} /$ I for IBM10 and $0.29 * 10^{9} / \mathrm{l}$ for IBM60 $(P=$ n.s. $)$. The median duration of leukopenia $\left(<1.00^{*} 10^{9} / \mathrm{I}\right)$ lasted $6 \mathrm{~d}$ for both groups (range: 4-11d, IBM10; 3-9d, IBM60) ( $P=$ n.s.). Median platelet nadir was $0 * 10^{9} / \mathrm{I}$ for both cohorts (IBM10: range $0.0-11.0^{*} 10^{9} / \mathrm{I}$, IBM60: $\left.0.0-1.0^{*} 10^{9} / \mathrm{I}\right)$. The periods of thrombocytopenia $\left(\leq 20.0^{*} 10^{9} / \mathrm{l}\right)$ were $5 \mathrm{~d}$ (IBM10) and 6d (IBM60; range 3-12d, both; $P=$ n.s.).

The results for early engraftment were set at the points in time $d+7, d+14, d+21, d+28$. Donor PBMC chimerisms were median 21\% (range: 8-34\%), 48\% (range: 29-53\%), 62\% (range: 55-73\%) and $67 \%$ (range: $47-73 \%$ ) in IBM10. The level of PBMC chimerism for IBM60 were 11\% (range: 0-34\%), 42\% (range: $20-48 \%$ ), $60 \%$ (range: $52-74 \%$ ) and $62 \%$ (range: $44-81 \%)(P=\mathrm{n}$. s., each). Donor granulocyte chimerisms were median $33 \%$ (range: $11-83 \%), 99 \%$ (range: $58-100 \%), 100 \%$ (range: $81-100 \%$ ) and $100 \%$ (range: $82-100 \%$ ) in IBM10, respectively. The granulocyte chimerism in IBM60 amounted to 34\% (range: 3$87 \%$ ), 96\% (range: $91-100 \%), 98 \%$ (range: $89-100 \%$ ) and $67 \%$ (range: $94-100 \%)(P=$ n.s., for all time points).

Conclusion: Our data suggest that modification of the HSC infusion rate has no impact on the early granulocyte and PBMC engraftment. Whether this is also true if grafts with low numbers are used has to be determined in subsequent studies.

Disclosure of Interest: None declared.

\section{AB76}

Hematopoietic stem cell transplantation for childhood leukemia in Mahak

A. A. Hedayati- Asl ${ }^{1, *}$, A. Mehrvar ${ }^{1}$, M. Tashvighi ${ }^{1}$, A. Naderi ${ }^{1}$, V. Fallah', R. Zangooei ${ }^{1}$

${ }^{1}$ MAHAK Cancer Children's Hospital, Tehran, Iran, Islamic Republic of

Introduction: The cure rate of childhood acute lymphoblastic leukemia (ALL) has improved considerably and approaches $80 \%$ today. However, the outcomes of patients who suffer from leukemic relapse remain unsatisfactory. Despite the high cure rate of children and adolescents with ALL a subgroup of patients benefit from allogeneic HSCT. Allo HSCT remains the standard treatment for intermediate/high risk AML patients.

Material (or patients) and methods: Forty-two patients, $\mathrm{ALL}=30$ and $\mathrm{AML}=12$ age 1 to 20 years with median age 11.5 years, $M / F=19 / 23(M / F A L L=14 / 16, A M L=5 / 7)$ underwent $S C T$ in our hospital (from 2012-2015). Forty-one patients transplanted Allo HSCT and 1 pt AML Auto HSCT. Conditioning regimens consisted of Busulfan (IV)+Cyclophosphamide for Allo and Cyclophosphamide+VP16 +Cytarabine for Auto HSCT. Peripheral blood (PB) was the source of progenitor cells in 34 patients, Bone marrow (BM) in 7 patients and cord blood in one patient. In Allo HSCT, 37 patient transplanted $6 / 6$ matched and 4 patients $5 / 6$ matched.GVHD prophylaxis regimen was cyclosporine+Mtx. All patients engrafted.

Results: In allogeneic PBSCT ALL patients' median time to absolute neutrophil count (ANC) $>0.5 \times 10^{9} / \mathrm{L}$ was 11 days, and the median time to platelet count $>20 \times 10^{9}$ was 14 days vs 18 and 22 days in Allo BM ALL patients. In allogeneic PBSCT AML patients median time to ANC $>0.5 \times 10^{9} / \mathrm{L}$ was 13 days, and the median time to platelet count $>20 \times 10^{9}$ was 15 days. (All patients with $A M L$ transplanted with $\mathrm{PB}$ ). At present 32 pts are alive (24 ALL, $8 \mathrm{AML}$ ) and 10 pts died due to ARDS, VOD, hemorrhagic stroke, sepsis and relapse. TRM was $9.5 \%$ at 100 days. Median time of death after transplantation was 165 days in ALL and 145 in AML. In Allo PBSCT ALL patients hospitalization period were 38 days vs. 46 in Allo BM ALL patients. Acute GVHD appeared in $80 \%$ pts. Chronic GVHD appeared in $48 \%$ pts. With a median follow-up of 15 months (3-41 months) after transplant the event-free survival were $74 \%$ and three years overall survival $78 \%$ in ALL patients. A median follow-up of 26.5 months (6-38months) after transplant the event-free survival were $70 \%$ and three years overall survival $67 \%$ in AML patients. 
Conclusion: Hematopoietic stem cell transplantation can lead to durable remissions in children and adolescents with leukemia and increase in survival of children. PBSCT in childhood ALL was consistent with significant faster ANC and platelet recovery in allogeneic PBSCT, hospitalization was shorter. Longer follow-up is required to evaluate fully efficacy and long-term results.

Disclosure of Interest: None declared.

\section{AB77}

Acute myeloid leukemia in a 19 year old patient previously treated for osteosarcoma - case report

C. Dragan ${ }^{1, *}$, M. Murat ${ }^{1}$, D. Georgescu ${ }^{1}$, M. Tevet ${ }^{1}$, F. Mihai $^{1}$

${ }^{1}$ Hematology, Colentina Clinical Hospital Bucharest, Bucharest, Romania

Introduction: As patients with osteosarcoma become longterm survivors, increasing attention has turned to the burden of late effects. Recent studies showed an increase in the incidence of secondary malignant neoplasms in patients with osteosarcoma compared with the general population. The risk of developing leukemia was reported to be in an increasing rate in the last decade. Different molecular and pathogenic agents may be implicated in the development of second malignant neoplasms in long-term survivors of osteosarcomas. Some authors believe that the treatment with topoisomerase II inhibitors may have a role in the development of $S A M L$, while others talk about cisplatin-associated SAML. ${ }^{[1,2,3]}$

Material (or patients) and methods: Case report. In this report we present the case of a 19 year old patient diagnosed in the Hematology Department of Colentina Clinical Hospital in February 2015 with Acute Myeloid Leukemia following the treatment (chemo- and radiotherapy) for an osteogenic osteosarcoma performed 3 years earlier.

Results: In this report we present the case of a 19 year old patient diagnosed in the Hematology Department of Colentina Clinical Hospital in February 2015 with Acute Myeloid Leukemia following the treatment (chemo- and radiotherapy) for an osteogenic osteosarcoma performed 3 years earlier. The cytogenetic examination revealed an abnormal karyotype with 91 chromosomes (XXY). The molecular biology exam did not find mutations in the FLT3ITD, FLT3D835 and NPM1 A genes. After the first induction course of chemotherapy the patient achieved complete remission, which was consolidated by 3 more courses of chemotherapy and an allogeneic stem cell transplant.

Conclusion: Considering the prior treatment for the osteosarcoma consisting of radiotherapy and chemotherapy we considered this to be a secondary Acute Myeloid Leukemia, this representing a major negative prognostic factor, along with the cytogenetic abnormalities found. These factors strongly indicate that the first complete remission should be consolidated by allogeneic stem cell transplant. The particularities of this case are the association of the two malignancies in a young patient, the presence of the abnormal karyotype (hyperploid), and also the good response of the patient to the induction therapy, achieving complete remission after the first induction course, and of course, sustaining that response.

References: 1 . Therapy-related acute myeloid leukemia with $\mathrm{t}(2 ; 11)(\mathrm{q} 37 ; \mathrm{q} 23)$ after treatment for osteosarcoma. Bielorai $\mathrm{B}$, Meyer C, Trakhtenbrot L, Golan H, Rozner E, Amariglio N, Izraeli S, Marschalek R, Toren A. Cancer Genet Cytogenet. 2010 Dec;203(2):288-91. doi: 10.1016/j.cancergencyto.2010.08.002. 2. Second malignant neoplasm in patients with osteosarcoma of the extremities treated with adjuvant and neoadjuvant chemotherapy. Bacci G, Ferrari C, Longhi A, Ferrari S, Forni C, Bacchini $P$, et al. J Pediatr Hematol Oncol. 2006;28:774-80.

3 . Secondary acute myeloid leukemia after successful treatment for osteosarcoma. Rakesh Mittal, N. V. Ramaswamy,1R. Pandita,1S. Al Bahar,1N. Khalifa, and S. Omar, Indian J Med Paediatr Oncol. 2010 Jan-Mar; 31(1): 33-35.

Disclosure of Interest: None declared.

\section{AB78}

Outcome of the pediatric patients with normal karyotype acute myeloid leukemia in Yeungnam region, South Korea: multi-center retrospective analysis

H. W. Chueh ${ }^{1, *}$, J. M. Lee ${ }^{2}$, K. S. Lee,, Y. T. Lim ${ }^{4}$, J. Y. Lim $^{5}$ E. S. Park , H. S. Kim ', Y. J. Shim ${ }^{6}$, E. J. Choi', J. Park ${ }^{8}$, J. O. Hah', J. A. Park ${ }^{10}$, S. K. Park ${ }^{11}$, J. Y. Kim ${ }^{3}$ on behalf of Yeongnam Pediatric Hematology Oncology Working Group

${ }^{1}$ Department of Pediatrics, Dong-A University Hospital, Busan, ${ }^{2}$ Department of Pediatrics, Yeungnam University, ${ }^{3}$ Department of Pediatrics, Kyungpook National University, Daegu, ${ }^{4}$ Department of Pediatrics, Busan National University, Busan, ${ }^{5}$ Department of Pediatrics, Gyeongsang National, Jinju, ${ }^{6}$ Department of Pediatrics, Keimyung University, 7 Department of Pediatrics, Daegu Catholic University, Daegu, ${ }^{8}$ Department of Pediatrics, Inje University Busan Paik Hospital, Busan, ${ }^{9}$ Department of Pediatrics, Fatima Hospital, Daegu, ${ }^{10}$ Department of Pediatrics, Inje University Haeundae Paik Hospital, Busan, ${ }^{11}$ Department of Pediatrics, Ulsan University, Ulsan, Korea, Republic Of

Introduction: Recent progress on genetics, many karyotypes and genetic information which can refer prognosis, and furthermore, guiding the future treatment, have been found in acute myeloid leukemia (AML). However, normal karyotype $A M L$, which consist almost half of the AML, doesn't have any conclusions about its prognosis. Therefore, in this study, outcome of the patients with normal karyotype AML in Yeungnam region of South Korea to make the guide for future treatment.

Material (or patients) and methods: Medical information of the patient who diagnosed and treated in Yeungnam region hospitals between 1st Jan 2000 and 1st Aug 2015 were collected and analyzed retrospectively.

Results: Total of 235 patients were diagnosed as AML in Yeungnam region, and the 197 patients received treatment at the hospital located in Yeungnam region. Among them, 36 patients were normal karyotype AML. 8 patients showed CR after $1 \mathrm{st}$ induction chemotherapy, but 26 patients achieved CR after all induction phase. Among the patient who achieved CR, 5 patients relapsed, and only 1 patient alieved. 14 patients received allogeneic hematopoietic stem cell transplantation (HSCT), and rest of them received only chemotherapy. 7 patients who received only chemotherapy were alive. The overall survival rate was $63.8 \%$, and the median survival time was 7.5 years. The overall survival of the patients who received allogeneic HSCT was $78.6 \%$, and the median survival time was 8.5 years. The survivals of the patients who received allogeneic HSCT superior significantly compared with that of the patients who did not received HSCT.

Conclusion: Patients with normal karyotype AML showed relatively good outcome. The survival rate of the patients who received allogeneic stem cell transplantation was significantly higher than that of the patients who did not. But due to the short-hand of this study, the roll of HSCT should be evaluated through further study.

Disclosure of Interest: None declared.

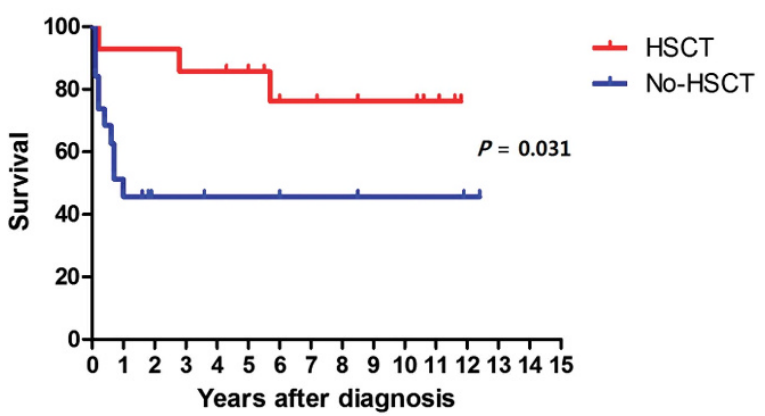


AB79

Treatment outcome of pediatric acute myeloid leukemia at Yeungnam region in South Korea - Multicenter retrospective study

J. M. Lee ${ }^{1, *}$, K. S. Kee ${ }^{2}$, J. Y. Kim ${ }^{2}$, J. Y. Lim ${ }^{3}$, E. S. Park ${ }^{3}$, H. S. Kim ${ }^{4}$ Y. J. Shim ${ }^{4}$, E. J. Choi ${ }^{5}$, H. Chueh ${ }^{6}$, J. Park', J. A. Park', S. K. Park', J. O. $\operatorname{Hah}^{10}$, Y. T. $\operatorname{Lim}^{1}$, Y. T. $\operatorname{Lim}^{11}$

${ }^{1}$ Pediatrics, Yeungnam University, ${ }^{2}$ Pediatrics, Kyungpook National University, Daegu, ${ }^{3}$ Pediatrics, Gyeongsang National University, Jinju, ${ }^{4}$ Pediatrics, Keimyung University, ${ }^{5}$ Pediatrics, Daegu Catholic University, Daegu, ${ }^{6}$ Pediatrics, Dong-A University, ${ }^{7}$ Pediatrics, Inje University Busan Paik Hospita, ${ }^{8}$ Pediatrics, Inje University Haeundae Paik Hospital, Busan, ${ }^{9}$ Pediatrics, Ulsan University, Ulsan, ${ }^{10}$ Pediatrics, Fatima Hospital, Daegu, ${ }^{11}$ Pediatrics, Busan National University, Busan, Korea, Republic Of

Introduction: Acute myeloid leukemia (AML) is second most common leukemia in children, accounting for $25-30 \%$ of childhood leukemia. Recently, intensive therapy with effective supportive care has increased survival rates to $70 \%$.

Material (or patients) and methods: We performed a retrospective study on patients with AML diagnosed from 2000 to 2013 Yeungnam region. Total of 263 patients were enrolled from 10 institutes.

Results: Age at diagnosis was $8.8 \pm 5.7$ years. Male to Female ratio was 1.48:1. In FAB classification, M2 (34.3\%) were most common, followed by M1 (14\%), M3 (11\%), M4 (11\%), M5 (11\%). M7 (5.5\%), and M0 (3\%). After induction, $77 \%$ of patients were achieved complete remission (CR). After first CR, hematopoietic stem cell transplantation (HSCT) was performed as consolidation in $54 \%$ of patients. The overall survival for patients who did not undergo transplantation was $73.2 \%$ whereas it was $75.62 \%$ for HSCT $(P=0.935)$. The 2 -year overall survival rate was $64.7 \%$. The 2 -year overall survival rate for patients who diagnosed before and after 2006 were $55.5 \%$ and $72.3 \%$, respectively $(P=0.049)$. $2-y r$ OS and EFS of AML in Yeungnam region is $62.5 \%, 58.3 \%$, respectively. The outcome has improved significantly after 2006. (OS 71.7\%, EFS 64.4\%). Multivariate analysis showed age under 2 years has poor outcome.

Conclusion: The clinical outcome of pediatric AML patient had been improved. The outcome has improved significantly after 2006. Age under $2 y$ has poor outcome. HLA-matched HSCT is an effective treatment for pediatric patients with intermediaterisk AML. Large prospective clinical trial is needed Disclosure of Interest: None declared.

AB80

Hematopoietic stem cell transplantation in AML patients; a battle to be won - 15 years single centre experience

L. Chadievski ${ }^{1, *}$, S. Genadieva Stavrik ${ }^{1}$, Z. Stojanoski ${ }^{1}$, A. Pivkova Veljanovska $^{1}$, D. Miloska ${ }^{1}$, L. Cevreska $^{1}$, B. Georgievski ${ }^{7}$

${ }^{1}$ University Clinic for Hematology, Skopje, Macedonia, The Former Yugoslav Republic Of

Introduction: Acute myeloid leukemia (AML) is a well-defined hematologic disorder that presents as a big challenge to all physicians. Can a doctor from the haematology department be a noble man confronting this disease? We represent a small bone marrow transplant unit that manifests a big,,struggle, during the past 15 years, achieving results that fuel up the battle for the future to more aggressive and more productive. Maybe, hematopoietic stem cell transplantation (HSCT) has the only curative potential in the therapeutic approach to this patients.

Material (or patients) and methods: During the last 15 years we have performed $360 \mathrm{HSCT}$. The gender distribution of our patients was males 184, and females 176 . The most frequent indication for HSCT was AML, representing $44 \%$ of all transplanted patients, or precisely 129 patients. In this group of patients, $78(60 \%)$ were autologous HSCT in first complete remission (CR), and $41(40 \%)$ allogeneic sibling HSCT. In the autologous setting, we used Bu-Cy conditioning regimen in 50 (62\%) patients, G-CSF+etoposide $2000 \mathrm{mg} / \mathrm{m}^{2}$ total dose in 39 (63\%) patients as standard mobilising regimen. Peripheral blood stem cells (PBSC) were used in 62 patients $(80 \%)$. The mean age at diagnosis was 41 years, we performed an average of 1.59 apheresis procedures, with the mean amount of $3.0 \times 10^{8} \mathrm{MNC}$. The average days to engraftment was 12 . In the allogeneic setting, the mean age to transplantation was 34 years. We used Bu-Cy conditioning regimen in 39 (76\%) patients, and Bu-Cy-Mel in $10(19 \%)$ patients. Acute GvHD was confirmed in 19 patients $(38 \%)$, and chronic in $16(31 \%) .32(63 \%)$ patients were transplanted in CR, and 19 (37\%) in active disease.

Results: The 5 year overall survival (OS) in AML patients treated with allogeneic sibling HSCT was $55 \%$. In term of the disease status before, those patients that were in CR, had 5 year OS of $73 \%$, despite those that were transplanted in active

[AB79]

\section{Treatment outcome (HSCT)}

\section{Overall Survival}
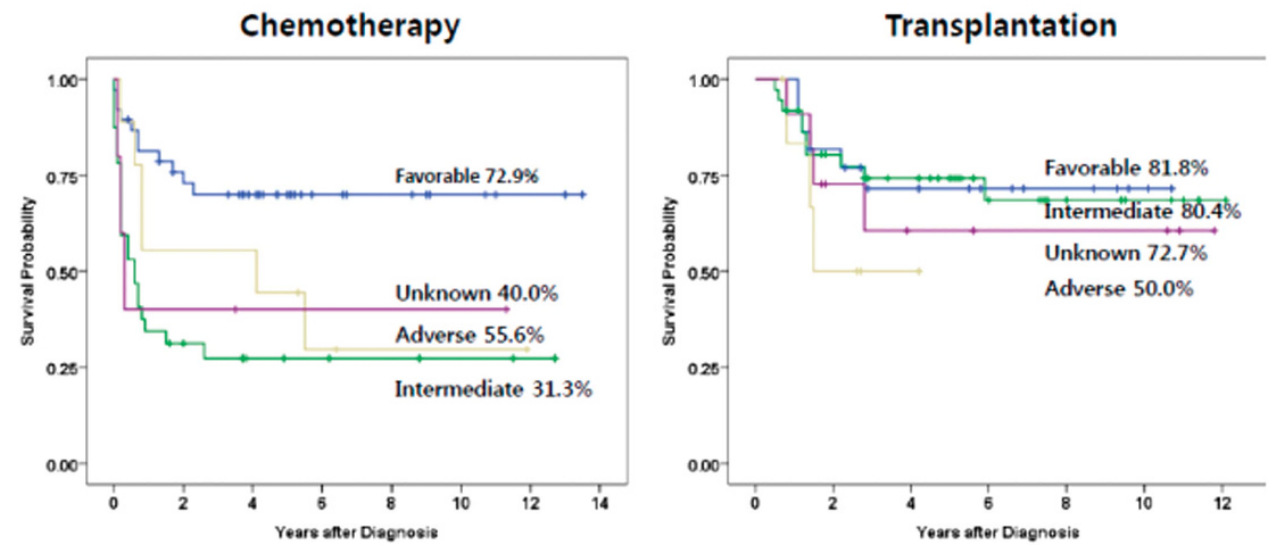
disease, with 5 year OS of only $15 \%$. In the autologous setting, the 5 year OS was $44 \%$. The main reason for mortality was relapse of the primary disease.

Conclusion: HSCT is a well-established, justified and proven therapeutic option for AML patients, especially for patients in $\mathrm{CR}$ and appropriate risk profile. Still there is much to be done, maybe on the field of the initial treatment, conditioning protocols, or the management of GvHD.

References: 1. Jemal A, Thomas A, Murray T, Thun M (2002). "Cancer statistics, 2002". CA Cancer J Clin 52 (1): 23-47;

2. Biggs JC, Horowitz MM, Gale RP, et al Bone marrow transplants may cure patients with acute leukemia never achieving remission with chemotherapy. Blood 80:1090, 1992; 3. Immunologic purging of autologous peripheral blood stem cell products based on CD34 and CD133 expression can be effectively and safely applied in half of the acute myeloid leukemia patients. Feller N1, van der Pol MA, Waaijman T, Weijers GW, Westra G, Ossenkoppele GJ, Schuurhuis GJ.;

Disclosure of Interest: None declared.

AB81

purely myeloablative conditioning regimen in aggressive multiple sclerosis: two case reports

C. Innocenti ${ }^{1, *}$, A. Barilaro ${ }^{2}$, A. Fani ${ }^{1}$, A. Mariottini ${ }^{2}$, L. Massacesi ${ }^{2}$, A. M. Repice, ${ }^{2}$. Saccardi ${ }^{1}$

${ }^{7}$ Hematology Dept, ${ }^{2}$ Neurology Dept, Careggi University Hospital, Florence, Italy

Introduction: High dose chemotherapy with autologous hematopoietic stem cell transplantation (aHSCT) is a promising approach for treatment of aggressive multiple sclerosis (MS). The most widely used conditioning regimens worldwide are the intermediate intensity BEAM+ATG and the low intensity cyclophosphamide/ATG, often reported as "Lymphoablative" as opposite to "Myeloablative" with reference to all the others. Outcome analysis of either single center series or Registry cohorts showed no advantage in using high intensity regimens, assuming that Myeloablative regimens are supposed to have a high transplant-related toxicity and a similar therapeutic impact on an Autoimmune Disease. We report here two successful aHSCT in very active MS forms using two intermediate intensity, myeloablative regimens.

Material (or patients) and methods: Case 1. A 33 years old woman with aggressive MS and advanced disability (EDSS 6.5) was enrolled in a transplant program and planned to receive a BEAM/ATG conditioning regimen. A severe anaphylactic reaction occurred after $\mathrm{BCNU}$ infusion, requiring her transfer to ICU. After her return to BMT Unit we decided to finalize the conditioning regimen with just Melphalan, without ATG.

Case 2. A 27 years old woman with relapsing remitting MS and moderate disability (EDSS 4.5) was also planned to receive a BEAM/ATG conditioning regimen. The rectal swamp collected upon admission showed a Carbapenems-Resistant Klebsiella Pneumoniae (KPC); therefore we decided to exclude melphalan and ATG from the conditioning regimen.

Results: Complete engraftment occurred in a normal range for both patients;. no viral reactivations nor major adverse events after transplant were shown. Patients underwent periodic follow-up after HSCT for 6 years and 18 months, respectively. Both patients didn't show any clinical relapses, disability progression nor new lesions at MRI (NEDA3 negative); clinical assessment showed an EDSS score improvement (from 6.5 to 5.5 and from 4.5 to 4.0 , respectively) in both patients.

Conclusion: Despite the administration of a purely myeloablative regimen and the lack of ATG, both patients experienced a full neurological response and a very good hematological tolerability. The optimal conditioning regimen for MS is still to be defined.

Disclosure of Interest: None declared.

\section{AB82}

Immune neutropenia mimicking graft failure after granulocyte transfusions and allogeneic stem cell transplantation in a child severe congenital neutropenia B. Kremens ${ }^{1, *}$, O. Basu ${ }^{1}$, C. Kreissig ${ }^{2}$, R. Buescher ${ }^{3}$, U. Sachs ${ }^{4}$ ${ }^{1}$ Pediatric Hematology Oncology, University Hospitals of Essen, Essen, ${ }^{2}$ DRK (German Red Cross), Blutspendedienst West, Breitscheid, ${ }^{3}$ Department of Pediatric Nephrology, University Hospitals of Essen, Essen, ${ }^{4}$ Clinical Immunology and Transfusion Medicine, Justus Liebig University, Giessen, Germany

Introduction: Children with Severe Congenital Neutropenia (SCN), an inborn failure of granulopoiesis with recessive inheritance, suffer from life-threatening infections even under treatment with G-CSF. Allogeneic stem cell transplantation is the only curative measure.

Material (or patients) and methods: Case report A 2-year-old girl with SCN (heterogeneous missense mutation in exon 4 of ELANE gene) developed, although under treatment with G-CSF, an acute appendicitis with perforation. She survived under antibiotics, 3rd party donor granulocyte transfusions and after surgery. Her bone marrow cytology at that time showed an arrest of granulopoietic precursors at the level of promyelocytes, increased eosinophils and normal erythropoiesis and thrombopoiesis, the characteristic BM picture found in SCN. Having survived this event, she received peripheral hematopoietic stem cells from a 9/10 matched unrelated donor after conditioning with fludarabin, thiotepa, treosulfan and ATG at 2 years and 10 months of age. The graft was manipulated to contain $3.3 \times 10 \mathrm{E} 6 \mathrm{CD} 34+$ cells/kg and $8.4 \mathrm{x}$ $10 \mathrm{E} 6 \mathrm{CD} 3+/ \mathrm{kg}$. Ciclosporin A was given for GVHD prophylaxis, G-CSF was instituted from day +4).

Results: Hematopoietic recovery: for platelets $>20 / \mathrm{nl}$ day 11 ; $>50 / \mathrm{nl}$ day 12 ; for leukocytes $>1.0 / \mathrm{nl}$ day 12 . PMNs rose to $>0.5 / \mathrm{nl}$ on day 14 , reached a maximum of $4.6 / \mathrm{nl}$ on day 16 , and then declined to $0-0.01 / \mathrm{nl}$ on day 23 at which level they remained even under G-CSF treatment. Bone marrow cytology at this time showed exactly the same arrest of granulopoiesis at the level of promyelocytes, increased eosinophils and fully recovered erythro- and megakaryopoiesis as before SCT. However, graft failure was ruled out as the BM cells expressed $100 \%$ donor chimerism. In search of an etiology, antibodies against granulocyte antigen HNA-2 (CD 177) were detected at titers of $>1: 4,000$. Plasmapheresis was started 47 days post SCT. The titers decreased to $1: 2,000$ after the first, to $1: 2$ after the 4th, and to 1:1 or 0 after the 9th (and final) plasmapheresis. Peripheral PNM counts increased to $>1.0 / \mathrm{nl}$ after the 4 th plasmapheresis, oscillated somewhat during 3 weeks and remained within a range of 1.0 to $3.1 / \mathrm{nl}$ since then. The patient successfully tackled a skin aGvHD (stage 4), CMV reactivation, ADV infection and interstitial pneumonia, and is now alive, thriving and immunocompetent, at 3 years post SCT, enjoying stable PNM counts.

Conclusion: Granulocyte transfusions - needed to surmount an appendicitis - apparently led to immunization against HNA-2 in this child with SCN, either because she was one of the $3 \%$ individuals who lack this glycoprotein, or because she has never expressed it since the very beginning of her embryonal granulopoiesis due to her underlying SCN. The bone marrow cytologies of SCN and of alloimmune neutropenia looked undistinguishably similar. The complete chimerism led us to correctly diagnose and treat this post SCT enigma.

Disclosure of Interest: None declared. 


\section{AB83}

Mismatched Unrelated Donor Hematopoietic Stem Cell Transplant for Sickle Cell Dlsease

G. Kharya ${ }^{1, *}$, D. Doval ${ }^{1}$, D. choudary ${ }^{1}$, V. Khandelwal ${ }^{1}$, E. Kaul ${ }^{1}$ S. Lunkad ${ }^{1}$, K. Pessi ${ }^{1}$, A. Handoo', T. Dadu', G. Dhamija ${ }^{1}$, R. Setia ${ }^{1}$, B. Sharma ${ }^{1}$, S. Sharma ${ }^{1}$

${ }^{1}$ Hemato-oncology and BMT, BLK Super specialty hospital, Delhi, India

Introduction: Hematopoietic stem cell transplant (HSCT) can cure sickle cell disease (SCD). This option is however limited by availability of matched sibling (MSD) or related unaffected donors. Over the years results of alternative donor HSCT have increased significantly. Outcome of matched unrelated donor BMT for SCD is as good as MSD but literature on mismatched unrelated donor HSCT for SCD is scarce. We report a child with SCD with complications being successfully transplanted using mismatched (mismatch at A locus) unrelated donor.

Material (or patients) and methods: 18 year old boy with SCD on regular transfusions was taken up for mismatch unrelated donor HSCT in the absence of suitably matched sibling or unrelated donor. He was conditioned using busulfan $3.2 \mathrm{mg} / \mathrm{kg} /$ day $x 4$ days, cyclophosphamide $50 \mathrm{mg} / \mathrm{kg} /$ day $\mathrm{x}$ 4 days and hATG (pfizerATGAM) $30 \mathrm{mg} / \mathrm{kg} /$ day x 3 days. GVHD prophylaxis included cyclosporine and methotrexate. Patient received GCSF mobilized peripheral blood stem cells harvested from unrelated donor with a target MNC $>8 \times 10^{8} / \mathrm{kg}$. He received $9.03 \times 10^{\wedge} 8 / \mathrm{kg}$ mononuclear cells, $8.5 \times 10^{\wedge} 6 / \mathrm{kg}$ CD34 cells.

Results: Polymorphonuclear cell and platelet engraftment were seen on $\mathrm{D}+9$ and $\mathrm{D}+16$ respectively. Whole blood chimerism on day +21 showed $99.45 \%$ donor cells. He developed engraftment syndrome which was managed with steroids. He later suffered from reactivated CMV which was managed with ganciclovir. He was discharged on $\mathrm{D}+20$ in good clinical condition. He is currently $D+380$ post HSCT having limited oral GVH on tapering doses. Post HSCT his sickle percentage has gone down from $88 \%$ to $22 \%$.

Conclusion: Mismatched unrelated donor HSCT can be successfully done in the absence of suitably matched sibling or unrelated donor for SCD using myeloablative conditioning. Options of mismatched unrelated donor HSCT for SCD needs to be explored further.

Disclosure of Interest: None declared.

\section{AB84}

Autologous stem cell transplantation for children and adolescents with relapsed and refractory Hodgkin's lymphoma

A. A. Hedayati- Asl ${ }^{1, *}$, A. Mehrvar ${ }^{1}$, M. Faranoush ${ }^{1}$, P. Dinarooni ${ }^{1}$, R. Zangooei ${ }^{1}$

${ }^{1}$ MAHAK Cancer Children's Hospital, Tehran, Iran, Islamic Republic Of

Introduction: Despite the generally excellent prognosis of children and adolescents with Hodgkin's lymphoma, approximately $20 \%$ of patients relapse. High-dose chemotherapy followed by autologous stem cell transplantation (ASCT) is a recognized treatment option for patients with relapsed Hodgkin's lymphoma. This study evaluates the results and outcome of non-cryopreserved autologous stem cell transplant of 25 patients with Hodgkin lymphoma.

Material (or patients) and methods: Twenty-five patients age 5 to 25 years (median 15.5 years, $M / F=18 / 7$ ), with relapsed, refractory or poor prognosis $\mathrm{HD}$, underwent ASCT in our hospital (from 2012-2015). Status at transplant was: second complete remission (CR2): $n=13$; further $\mathrm{CR}(\mathrm{CR}>2): n=10$ partial remission (PR): $n=2$. Twenty-five patients received chemotherapy- based conditioning regimens: cyclophosphamide, carmustine and etoposide (CBV): 6, CCNU, etoposide, cytarabine and melphalan (CEAM): 19, Peripheral blood (PB) was the source of progenitor cells in 25 patients. All patients engrafted.
Results: The median mononuclear cell dose was $5.8 \times 10^{8} / \mathrm{kg}$. The median time to reach absolute neutrophil count $>0.5 \times$ $10^{9} / \mathrm{L}$ was 12 days, and the median time to platelet count $>20 \times 10^{9}$ was 14 days. Grade 2 and grade 3 mucositis was seen in $65 \%$ of our patients. Transplant-related mortality at 100 days not occurred. Only two patient relapsed 15 and 18 months after transplant (mean $16.5 \mathrm{~m}$.). With a median follow-up of 32 months (5-41 months) after transplant the event free survival were $87 \%$. Only one patient had death, two years after transplantation. No significant different between CBV group vs. CEAM group in engraftment day.

Conclusion: High-dose therapy with stem cell rescue can lead to durable remissions in children and adolescents with advanced HD. Future investigations should focus on strategies designed to decrease relapse after auto-transplantation, particularly in patients at high risk for relapse. Our analysis suggests that these regimens (CEAM, CBV) are feasible in pediatric patients with acceptable engraftment and toxicity.

Disclosure of Interest: None declared.

\section{AB85}

Chronic Active EBV (T-Cell subtype) infection successfully treated with Flu-Mel-Cam unrelated stem cell transplantation

C. Andrews' ${ }^{1}$, C. Ryan ${ }^{2}$, L. Bacon ${ }^{1}$, M. Jeffers' ${ }^{2}$, E. Vandenberghe ${ }^{1, *}$ ${ }^{1}$ Haematology, ${ }^{2}$ Pathology, St. James's Hospital, Dublin, Ireland

Introduction: Chronic active EBV (CAEBV) is a rare life threatening disorder which presents with fever, lymphadenopathy and splenomegaly. It is caused by severe immune dysregulation and clonal proliferation of B or T-lymphocytes following EBV infection in the absence of underlying immunodeficiency. It can only be cured by allogeneic SCT but this is only in $50 \%$ of cases (1). We report the successful treatment of a patient with CAEBV (T-subtype).

Material (or patients) and methods: Case report: A 19 year old previously well female was referred with an ECOG of 3 and 9 week history of pyrexia, weight loss and abdominal pain. This was secondary to hepatosplenomegaly, mild pancytopenia $\left(\mathrm{Hb} 8.4 \mathrm{~g} / \mathrm{L}\right.$, WCC $2.05 \times 10^{9} / \mathrm{L}$, Neutrophils $1.35 \times 10^{9} / \mathrm{L}$ and Platelets of $134 \times 10^{9} / \mathrm{L}$ ), abnormal LFTs (Bilirubin $17 \mathrm{mg} / \mathrm{dl}$, ALT 341 IU/L, Alk Phos 1675IU/L) and an LDH of 2086IU/L. Infection work up was negative apart from a positive EBV lgG. Her EBV DNA viral load was $>3.5 \times 10^{6}$. She was commenced on prednisolone and valganciclovir for four weeks for suspected infectious mononucleosis prior to referral with no improvement.

A CT thorax/abdomen/pelvis confirmed hepatosplenomegaly and $1 \mathrm{~cm}$ mesenteric/periportal adenopathy. A bone marrow aspirate and biopsy was performed which showed trilineage haematopoiesis with reactive features. The mesenteric node and liver were biopsied and were consistent with CAEBV, T-cell subtype with EBER positive T-lymphocytes and clonality confirmed by TCR beta and gamma rearrangement. Haemophagocytic lymphohistiocytosis was excluded as she had a ferritin $<800 \mathrm{ng} / \mathrm{ml}$, normal triglycerides and had no evidence of haemophagocytosis in the biopsies.

Results: She was treated with a hybrid protocol of gemcitabine, etoposide and dexamethasone for 4 cycles because of her poor performance status and abnormal LFTs and achieved a PR with improvement in LFTs, blood count, scan and undetectable EBV DNA. She was consolidated with a reduced intensity matched unrelated SCT using fludarabine, busulphan and alemtuzumab conditioning resulting in engraftment at day 14 . She had grade one skin graft versus host disease but no post-transplant EBV reactivation. She had LFT normalization after six months and remains well eighteen months post- transplantation.

Conclusion: We have presented this case to raise awareness of a rare manifestation of EBV infection and effectiveness of allo-SCT in its treatment (1). This case is unusual in that the patient had serological evidence of previous EBV infection with an unknown re-activation trigger, whereas it is usually triggered by a de novo infection. 
We are grateful to Dr E Jaffe $(\mathrm{NCl})$ for confirming the diagnosis of CAEBV and Dr J Cohen ( $\mathrm{NCl}$ ) for clinical management advice. References: (1) Characterization and treatment of chronic active Epstein-Barr virus disease: a 28 year experience in the United States. Cohen et al. Blood 2011.

Disclosure of Interest: None declared.

\section{AB86}

Salvage Therapy with Allogeneic Hematopoietic Cell Transplantation For Relapsed/Refractory Aggressive B-Cell Lymphomas: A Single-Center Experience

D. Dörfel ${ }^{1,2, *}$, S. P. Haen ${ }^{1}$, W. Vogel', L. Kanz ${ }^{1}$, R. Möhle ${ }^{1}$, S. Wirths ${ }^{1}$, C. Faul ${ }^{1}$, W. A. Bethge

${ }^{1}$ Department of Hematology and Oncology, University of Tuebingen, ${ }^{2}$ Clinical Collaboration Unit Translational Immunology, German Cancer Consortium (DKTK) and German Cancer Research Center (DKFZ), Partner site Tuebingen, Tuebingen, Germany

Introduction: Patients with relapse of high grade lymphoma after autologous transplantation or with primary refractory disease have a dismal prognosis. Allogeneic hematopoietic cell transplantation (allo-HCT) is a potentially curative salvage treatment but data on outcome with this approach are limited.

Material (or patients) and methods: We retrospectively analyzed all adult patients with high-grade lymphoma who underwent allo-HCT at our center between 2002 and 2013.

Results: A total of 38 adult patients received allo-HCT during this period. Histologies were diffuse large B cell lymphoma (DLBCL, $n=18)$, grey zone lymphoma $(n=2)$, and transformation from indolent lymphoma ( $\mathrm{FL}, n=9 ; \mathrm{CLL}, n=8$; marginal zone lymphoma, $n=1)$. The median age at time of allo-HCT was 46 (range, 28-68) years. 68\% ( $n=26)$ of patients had received an autologous transplantation prior to allo-HCT. If allo-HCT was used as primary transplantation strategy, refractory disease or inadequate mobilization of hematopoietic stem cells was present. Prior to allo-HCT patients were treated with a median of 5 chemotherapeutic regimens (range, $1-8)$ and $87 \%(n=33)$ of patients received rituximab prior to allo-SCT. At start of conditioning therapy, $32 \%$ of patients had refractory disease and $37 \%$ were in complete remission. Myeloablative conditioning regimens (MAC) were used in $45 \%$ of patients and $55 \%$ were treated with reduced intensity conditioning (RIC). There were no differences in median HCT-Cl score between RIC and MAC group (range 0-4). The median age for patients receiving MAC and RIC was 44 years and 54 years, respectively. Peripheral blood stem cells were exclusively used as graft source. Donors were matched related (MRD, $n=16)$, matched unrelated (MUD, $n=16$ ), mismatched unrelated (MMUD, $n=4)$, or haploidentical $(n=2)$. Median follow-up of patients alive was 64 months with a Kaplan Meier estimated overall survival (OS) for all patients of $53 \%, 47 \%$ and $36 \%$ and a progression-free survival (PFS) of $47 \%$, $35 \%$, and $32 \%$ at 1,2 , and 5 years. Transformation in high grade lymphoma did not result in inferior OS $(P=0.37)$. The use of RIC regimen resulted in significantly better median survival rates irrespective of disease risk index (MAC 2 months vs RIC 52 months, $P=0.01)$. The non-relapse mortality (NRM) at day 100,1 year and 3 years was $18 \%, 26 \%$ and $31 \%$. NRM after 1 year was higher after MAC with $35 \%$ compared to $14 \%$ after RIC. The cumulative incidence of acute $\mathrm{GvHD} \geq \mathbb{I}^{\circ}$ was $18 \%$ and of chronic GvHD 37\% (limited 21\%, extensive 16\%). After RIC patients had increased rates of limited chronic GvHD with $28 \%$ vs. only $11 \%$ in the MAC group. Rates for extensive chronic GvHD were similar with $18 \%$ vs. $14 \%$. Rates for limited chronic GvHD were $13 \%, 33 \%$ and $25 \%$ in the MRD, MUD and MMUD group. Extensive chronic GvHD was observed in 19\% and $13 \%$ in the MRD and MUD group.

Conclusion: Allogeneic HCT is a potentially curative treatment option even in advanced and refractory patients with a long term OS of $36 \%$ and PFS of $32 \%$ regardless of conditioning regimen with RIC resulting in significantly better OS.

Disclosure of Interest: None declared.
AB87

Early metabolic remission post autologous hematopoietic transplantation in Hodgkin Lymphoma conditioned with either BEAM or Busulfan-based regimen is correlated with significantly superior disease free survival

D. Mallouri ${ }^{1, *}$, V. Constantinou ${ }^{1}$, M. Iskas ${ }^{1}$, I. Batsis ${ }^{7}$ S. Papadimitriou' ', A. K. Panteliadou' ', V. Kalaitzidou' , A. Bouinta' S. Papaemmanouil ${ }^{2}$ P. Palladas ${ }^{3}$, I. Datseris ${ }^{4}$, A. Anagnostopoulos ${ }^{1}$, I. Sakellari ${ }^{1}$

${ }^{1}$ Haematology department - BMT unit, ${ }^{2}$ Pathology Department, ${ }^{3}$ Radiology Department, George Papanicolaou Hospital, Thessaloniki, ${ }^{4}$ Nuclear Medicine and PET/CT Department, Evangelismos General Hospital, Athens, Greece

Introduction: Fluorine-18-Deoxyglucose Positron Emission Tomography Scan (PET) is a valuable diagnostic tool in Hodgkin lymphoma (HL). In the autologous haematopoietic cell transplantation (AHCT) setting, the prognostic value of pre-AHCT PET has been established by current studies, but the significance of early post-AHCT PET negativity has not yet been fully investigated.

Material (or patients) and methods: In this retrospective, single center analysis, we evaluated the role of early postAHCT ( +3 to +6 months) metabolic remission in 58 consecutive HL patients (pts), aged 36 (13-62), who underwent AHCT during 2009-2014, for primary refractory (43), or relapsed disease (15) post 3 (1-9) lines of treatment. Disease status was chemosensitive to salvage treatment in 29 pts. The conditioning regimen was BEAM (17) or Busulfan-based (Busulfan $9.6 \mathrm{mg} / \mathrm{kg}$, Etoposide $400 \mathrm{mg} / \mathrm{m}^{2}$ and Melphalan $140 \mathrm{mg} / \mathrm{m}^{2}$, BuEM) (41).

Results: Forty eight pts were evaluated by PET at +3 to +6 months $(\mathrm{m})$ while the rest had disease progression by $C T$ assessment (7 received additional involved field irradiation post$\mathrm{AHCT}$, as part of the treatment plan). Thirteen pts had positive and 35 negative PET. The overall early-response rate by PETevaluation was $73 \%$ (35/48 evaluated pts). In univariate analysis, overall survival (OS) and disease free survival (DFS) were superior in pts achieving early post-AHCT negativity (5y-OS $89 \%$ vs $29 \%$; $P=0.007,5 y-D F S 78 \%$ vs $22 \% ; P=0.012$ ). Patients' characteristics according to the conditioning regimen were similar in terms of disease phase and chemosensitivity ( $x^{2}$ test). With a median follow up of 29 (2-76) m (BEAM 18, BuEM $45 \mathrm{~m}$ ), the estimated 5year DFS and time to progression (TTP) were similar in both conditionings. At last follow-up 40/48 (83\%) pts were alive and 32 $(66 \%)$ in CR. Treatment related mortality was low with both regimens (1\%). Overall survival was high and similar in both conditioning groups, with no significant difference in DFS. In multivariate analysis disease phase, chemosensitivity, type of conditioning and early-PET evaluation were incorporated. Early PET-negativity was the only significant factor correlated with superior OS $(P=0.02)$. In terms of DFS, disease chemosensitivity identified as significant favorable factor $(P=0.02)$, while early metabolic remission was of borderline significance $(P=0.06)$.

Conclusion: With the limitations of this retrospective analysis, early metabolic complete remission, along with disease chemosensitivity to salvage treatment, seems to be the most significant predictor in $\mathrm{HL}$ pts for improved overall survival and time to disease progression. Both conditioning regimens proved to be similarly efficacious and feasible, remaining the key players for a successful outcome of AHCT in lymphomas. Additionally, early PET can optimize the outcome in $\mathrm{HL}$ patients regarding the need for post AHCT consolidation in the era of novel targeted agents.

References: Ulaner GA, Goldman DA, Sauter CS et al. Prognostic Value of FDG PET/CT before Allogeneic and Autologous Stem Cell Transplantation for Aggressive Lymphoma. Radiology 2015.

Sakellari I, Mallouri D, Batsis I et al. Busulfex, etoposide and melphalan conditioning regimen for autologous hematopoietic cell transplant: a retrospective matched-pair analysis in advanced Hodgkin and non-Hodgkin lymphomas. Leuk Lymphoma 2015, 56(11):3071-81.

Disclosure of Interest: None declared. 


\section{AB88}

The role of hematopoietic stem cell transplantation for pediatric relapsed or refractory non-Hodgkin's lymphoma: Results of the Turkish Pediatric Bone Marrow Transplantation Study Group

V. Hazar 1,* V. Kesik', G. Tezcan Karasu ${ }^{3}$, G. Öztürk ${ }^{4}$, A. Küpesiz S. Çakı Kılıç ${ }^{3}$ E. Ataş ${ }^{2}$, V. Uygun ${ }^{6}$, N. Eker ${ }^{5}$, F. Erbey ${ }^{4}$, S. Yılmaz Bengoa', S. Emir $^{8}$, S. Anak ${ }^{9}$, H. Öniz ${ }^{10}$, H. Daloğlu ${ }^{6}$, S. Aksoylar ${ }^{11}$, Ü. Koçak ${ }^{12}{ }^{3}$ A. Tanyeli ${ }^{13}$, M. Karakükcü̈ ${ }^{14}, M$. Elli ${ }^{15}$, N. Kurucu ${ }^{16}$, A. Yeşilipek ${ }^{3}$ on behalf of on behalf of the Turkish Pediatric Bone Marrow Transplantation Study Group

${ }^{1}$ Pediatric Hematology/Oncology and BMT Unit, Medipol University Faculty of Medicine, Medipol Mega Hospital, Istanbul,

${ }^{2}$ Pediatric Oncology and BMT Unit, Gülhane Military Medical Academy, Ankara, ${ }^{3}$ Pediatric Hematology/Oncology and BMT Unit, Bahçeșehir University Faculty of Medicine, Göztepe Medical Park Hospital, ${ }^{4}$ Pediatric Hematology/Oncology and BMT Unit, Aclbadem University Faculty of Medicine, Acıbadem Atakent Hospital, Istanbul, ${ }^{5}$ Pediatric Hematology/Oncology and BMT Unit, Akdeniz University Faculty of Medicine, ${ }^{6}$ Pediatric BMT Unit, Bahçeșehir University Faculty of Medicine, Antalya Medical Park Hospital, Antalya, 'Pediatric Hematology and BMT Unit, Dokuz Eylül University Faculty of Medicine, Izmir, ${ }^{8}$ Pediatric Hematology/Oncology and BMT Unit, Ankara Child Health and Diseases Hematology and Oncology Training and Research Hospital, Ankara, ${ }^{9}$ Pediatric Hematology/Oncology and BMT Unit, Istanbul University Istanbul Faculty of Medicine, Istanbul, ${ }^{10}$ Pediatric Oncology and BMT Unit, Tepecik Child Health and Diseases Training and Research Hospital, ${ }^{11}$ Pediatric Hematology/Oncology and BMT Unit, Ege University Faculty of Medicine, Izmir, ${ }^{12}$ Pediatric Hematology/Oncology and BMT Unit, Gazi University Faculty of Medicine, Ankara, ${ }^{13}$ Pediatric Oncology and BMT Unit, Çukurova University Faculty of Medicine, Adana, ${ }^{14}$ Pediatric Hematology and BMT Unit, Erciyes University Faculty of Medicine, Kayseri, ${ }^{15}$ Pediatric Hematology/Oncology and BMT Unit, On Dokuz Mayıs University Faculty of Medicine, Samsun, ${ }^{16}$ Pediatric Oncology and BMT Unit, Oncology Training and Research Hospital, Ankara, Turkey

Introduction: High-dose chemotherapy and either autologous or allogeneic hematopoietic stem cell transplantation (HSCT) is one of the considered treatment options for patients with relapsed or refractory non-Hodgkin's lymphoma (NHL) but there are limited data about this issue in the pediatric setting Material (or patients) and methods: We examined the role of HSCT for patients aged less than or equal to 18 years at the time of transplantation with relapsed or refractory lymphoblastic $(n=24)$, Burkitt (12), diffuse large B cell (13) and anaplastic large cell lymphoma $(n=17)$, receiving autologous $(n=38)$ or allogeneic $(n=28) \quad(n=20$ matched sibling/family, $n=5$ unrelated and $n=3$ mismatched related donor) registered at the Turkish Pediatric Bone Marrow Registry. Risk factors affecting survival were evaluated using stratified Cox regression. Fifty-seven patients $(84 \%)$ had chemo-sensitive disease at the time of transplantation.

Results: Overall survival (OS) and event-free survival (EFS) were $68 \%$ and $59 \%$ with a median follow-up of 39 months; OS and EFS according to histopathological classification were $51 \%$ and $49 \%$ for lymphoblastic, $67 \%$ and $67 \%$ for Burkitt, $\% 86$ and $65 \%$ for anaplastic large cell and, $77 \%$ and $61 \%$ for diffuse large cell lymphoma, respectively. The outcomes were similar in regard of both OS and EFS in patients receiving autologous and allogeneic HSCT $(68 \%$ vs $70 \%$ and $55 \%$ vs $62 \%$, respectively). Both OS and EFS among patients with chemosensitive disease at the time of HSCT were significantly higher than those in patients with chemo-resistant disease $(74 \%$ vs $33 \%, P=0.001 ; 71 \%$ vs $22 \%, P<0.001$, respectively). Multivariate analysis showed that chemoresistant disease at the time of transplantation was the only factor predicting limited both OS (hazard ratio $=5.46$ ) and DFS (hazard ratio $=4.73$ ).
Conclusion: Intensive chemotherapy followed by either allogeneic or autologous HSCT is an effective strategy for children with relapsed or refractory $\mathrm{NHL}$ and offers durable disease free survival for a significant group of pediatric patients, especially for patients with chemosensitive disease at the time of transplantation.

References:

1. Gross TG, Hale GA, He W, et al. Hematopoietic stem cell transplantation for refractory or recurrent non-Hodgkin lymphoma in children and adolescents. Biol Blood Marrow Transplant 2010; 16: 223-230.

2. Harris RE, Termuhlen AM, Smith LM, et al. Biol Blood Marrow Transplant 2011; 17: 249-258.

3. Giulino-Roth L, Ricafort R, Kernan NA, et al. Pediatr Blood Cancer 2013; 60: 2018-2024.

Disclosure of Interest: None declared.

\section{AB89}

Outcome of autologous stem cell transplantation on aggressive non-Hodgkin lymphoma- a single center retrospective analysis

Y. Liu' ${ }^{1}$ X.-H. Chen', J.-L. Jia-Li', C. Zhang ${ }^{1}$, L. Gao', L. Gao', X. Zhang ${ }^{i, *}$

${ }^{7}$ Department of hematology, Xinqiao Hospital, Third Military Medical University, Chongqing, China

Introduction: Retrospectively analyzethe role of sequential autologous stem cell transplantation (ASCT) in aggressive NHL.

Material (or patients) and methods: 121 newly diagnosed patients with aggressive NHL were included from March 2007 to December 2013 in our center. Before proceeding transplantation, cases of patients in CR1 are 65, 28 cases of CR2, 18 cases of PR and 10 cases of NR. All patients were been treated by chemotherapy of three times (CTOD or MOED) plus highdose MTX $\left(1-3 \mathrm{~g} / \mathrm{m}^{2}\right)$ before transplantation. CEAC is considered as the preparative regimen that including additional local supplementary radical radiotherapy for patients with large mediastinal masses. The total four weeks biological treatment including five days of IL-2 and two days of rest, that was applied for the patients with hematopoietic recovery. The patients who showed $\mathrm{CR}$ before and after transplantation adopted consolidation treatment every three months, of which total courses are 4 to 6 and 6 to 8 . Patients who showed PR following transplantation accepted autologous transplantation in 3 months after transplantation. Patients who presented NR or RE accepted allogeneic transplantation.

Results: All patients acquired hematopoietic reconstitution, in which the average reconstituted time of ANC was $+11.6 \mathrm{~d}$ and PLT was $+14.2 \mathrm{~d}$ respectively. The main complications are tolerable, including gastrointestinal reactions $(82.9 \%$ of incidence rate), oral mucositis (51.2\% of incidence rate) and mild liver damage (19.55\% of incidence rate) which does not affect the progress of sequential treatment. The median follow-up time was 54 months (range 6-97 months). Among CR1 survivors, 55 patients are disease-free (84.6\%) and 10 survivors with disease $(15.6 \%)$ and no death; 20 CR2 survivors are disease-free $(71.4 \%), 15$ CR2 survivors with disease $(15.2 \%)$ and 5 deaths (17.8\%); 8 PR survivors are disease-free (61.5\%), 2 PR survivors in disease (15.4\%) and 3 deaths (23.1\%); 2 NR survivors are disease-free (20\%), 3 NR survivors with disease (30\%) and 5 deaths (50\%). The overall survival and progression-free survival of 5 years for all patients are $78.9 \%$ (95\% Cl 48.1-78.5\%) and 65.3\% (95\% Cl 52.3-74.6\%), respectively.

Conclusion: Sequential ASCT showed its efficacy in treatment for aggressive non-Hodgkin's lymphoma by increasing the cure rate of disease.

Disclosure of Interest: None declared. 


\section{AB90}

Tandem auto transplants of peripheral blood stem cells for lymphoblastic lymphoma: a single center retrospective study

J.-L. Li $^{1}$, Q. Wen ${ }^{1}$, L. Zhu ${ }^{1}$, X. Xiang ${ }^{1}$, L. Gao ${ }^{1}$, L. Gao ${ }^{1}$, D. Zeng ${ }^{1}$, C. Zhang ${ }^{1}$, P. Kong $P^{1}$, H. Liu ${ }^{1}$, X. Zhang ${ }^{1, *}$

${ }^{1}$ Department of hemaotlogy, Xinqiao Hospital,Third Military Medical University, Chongqing, China

Introduction: In order to evaluate the effecacy and safety of tandem autologous peripheral blood stem cell transplants (APSCT) for lymphoblastic lymphoma.

Material (or patients) and methods: The clinic data of 21 cases with lymphoblastic lymphoma in Xinqiao hospital were analyzed. The patients were classified by Ann Arbor staging system, including 5 stage III cases, 16 stage IV cases. Before the first transplantation, 16 cases were achieved complete remission and 5 cases achieved partial remission. Mobilizetion regimen was Mit+Vds+VP16+Dex+MTX combined with G-CSF $5 \mathrm{ug} /(\mathrm{kg} . \mathrm{d})$. The conditioning regimens for the tandem transplantation were CCNU+VP-16+Ara-c+CTX and IDA+Ara-C + CTX respectively. The interval of the tandem transplantation was 4 to 6 months. In tandem transplantation, the cells of MNC transfused were $4.3 \times 10^{8} / \mathrm{kg}(2.5 \sim 8.4) \times 10^{8} / \mathrm{kg}$ and $3.8(2.2 \sim 9.4) \times 10^{8} / \mathrm{kg} ; \mathrm{CD} 34^{+}$cells were $2.9(1.8 \sim 7.0) \times 10^{6} / \mathrm{kg}$ and $2.5(1.6 \sim 7.8) \times 10^{6} / \mathrm{kg}$ respectively $(P>0.05)$.

Results: All patients obtained prompt and sustained hematopoietic reconstitution. The interval of ANC $\geq 0.5 \times 10^{9} / \mathrm{L}$ was $+12 \mathrm{~d}(+9 \sim+21 \mathrm{~d})$ days and $+14 \mathrm{~d}(+10 \sim+17 \mathrm{~d})$ days; platelet $\geq 20 \times 10^{9} / \mathrm{L}$ was $+13 \mathrm{~d}(+9 \sim+31 \mathrm{~d})$ days and $+10 \mathrm{~d}(+8 \sim+50 \mathrm{~d})$ days, respectively $(P>0.05)$. After the tandem transplants, 3 cases relapsed, among 2 case died of recurrence, 1 case died of disease progression, 1 case died of transplantation related mortality, 12 cases were still in disease-free survival, 4 remained stable after median followup of 24 (2 81) months. The 3 -years disease-free survival (DFS) was $68.9 \%$. The 3 -years overall survival (OS) was $72.6 \%$. The main complication of transplantation were fever (76.2\%) and gastrointestinal symptoms (nausea (66.7\%) and vomiting (33.3\%)). There was no transplantation related mortality.

Conclusion: The tandam APSCT was safe and effective for lymphoblastic lymphoma.

Disclosure of Interest: None declared.

AB91

Experience on treatment outcome of transplant-eligible myeloma patients in a resource limited center in Asia

T. Jenq Tzong ${ }^{1}$, O. Tee Chuan ${ }^{1}$, Z. Z. Muhd ${ }^{1}$, L. Pek Kuen', J. Suriar ${ }^{1}$, C. Kian Meng ${ }^{1}$, T. Sen Mui ${ }^{1, *}$

${ }^{1}$ hematology, Hospital Ampang, selangor, Malaysia

Introduction: High-dose chemotherapy with autologous stem cell transplantation (ASCT) remains the standard of care for patients with multiple myeloma (MM) in Malaysia despite the promising development of novel agents. Here we present the treatment outcomes of our MM patients who have undergone high-dose chemotherapy with ASCT for the last 13 years with different induction regimens.

Material (or patients) and methods: We retrospectively analyzed a total of 156 patients who received high-dose melphalan (HD-MEL) followed by ASCT from August 2002 till October 2015 in our centre.

Results: The majority of the patients were from the Malay ethnicity, comprising of $56.41 \%$ of the cohort. Most of our patients were above 50 years old with $49.36 \%$ in the 50-59 years age-group and $21.79 \%$ aged above 60 years respectively. The induction strategy prior to ASCT has evolved from VAD regimen accounting for $15.38 \%$ to Thalidomide(T)based or Velcade (bortezomib) (V)-based regimens totaling $77.56 \%$ with time. About $7.05 \%$ of the patients had dual-novel agents (both thalidomide and Velcade) as induction treatment prior to ASCT. There were no significant age differences among the 4 groups. The Velcade-based and dual-novel agent groups had more male patients, accounting for $75 \%$ and $64 \%$ respectively. A majority had IgG subtype MM (VAD 61\%, Thalidomide-based: $73 \%$, Velcade-based: $70 \%$ and dual-novel agent: $80 \%$ ). At least $2 / 3$ of patients in all 4 groups achieved VGPR after induction with the highest $\mathrm{CR} / \mathrm{nCR}$ among patients receiving dual-novel agents at induction. More patients in the Thalidomide-based, Velcade-based group and dual-novel group attained $\mathrm{CR} / \mathrm{nCR}(26 \%, 29 \%$ and $36 \%$ respectively) as compared to VAD regimen (22\%) prior to ASCT. All patients successfully underwent PBSC mobilization and proceed with ASCT. The median day of neutrophils engraftment was 1 day earlier for Thalidomide- and Velcade-based as well as dualnovel group as compared to VAD for which median engraftment was Day+11. The incidence of TRM was $2 \%$. The overall survival (OS) of all MM patients from the time of diagnosis was $50 \%$ at 86 months. The median survival post transplant was 66 months from the time of transplant.

Conclusion: ASCT remains a relevant treatment option to deepen the response post induction and to prolong survival of MM patients in resource-limited settings such as Malaysia as compared to maintenance with new novel agents which are not as easily accessible as compared to ASCT.

Disclosure of Interest: None declared.

\section{AB92}

Comparison of double transplantation- tandem transplantation or transplantation in relapse - in patients with multiple myeloma

A. Jungova ${ }^{1, *}$, S. Vokurka ${ }^{1}$, M. Schutzova ${ }^{1}$, K. Steinerova ${ }^{1}$, L. Mohammadova', M. Karas' ${ }^{1}$, D. Lysak ${ }^{1}$, P. Jindra

${ }^{1}$ Hemato-oncology department, Charles University Hospital Pilsen, Pilsen, Czech Republic

Introduction: Multiple myeloma (MM) is malignant haematological disease affecting up to 600 people a year in the Czech Republic. Treatment options are constantly improved but autologous transplantation (Tx) remains an integral part of treatment protocols despite the development of modern drugs. Profit in using two autologous $T x$ versus single $T x$ is documented but without a recommendation of time interval between these two Tx. We compared 2 groups of patients with $2 \mathrm{Tx}$ in our study - in the first group (28 patients) was performed second Tx calculatedly on reaching PR and worse after induction in median of 5.5 months $(2-11)$. In the second group (38 patients) was performed second $\mathrm{Tx}$ in relapse without previous re-induction in median 8 months (4-8). Subanalysis divided this group in 2 subgroups according value of paraprotein before the second Tx (cutt of for ... low level" of paraprotein was $10 \mathrm{~g} / \mathrm{l}$,event. $1000 \mathrm{mg} / \mathrm{l}$ in MM with light chains), other findings were taken as "high level" including 3 patients with extramedullary disease. Other parameters were comparable (age, type of induction treatment, cytogenetic risk) without statistic difference in both groups.

Material (or patients) and methods: Retrospective analysis of patients with $\mathrm{MM}$ in order to compare time to initiation other treatment after last autologous Tx (TFS), overall survival (OS) in two different groups and baseline level of paraprotein by second $T x$ in patients transplanted in relapse.

Results: The analysed group consisted of 66 patients. Group was divided in two parts. In patients in the first part autologous Tx was performed as tandem Tx $(n=28)$, in the second part was until the time of relapse $(n=38)$. Median follow-up 46.5 months (21 - 165) vs. 33 months (20 - 112) $P=0.36$. In October 2015 lived 34/66 patients (52\%), died 32 patients $(48 \%)$. Median age in both groups is 59 years $(P=0.36)$, representation of genders - the ratio of men/ women $14 / 14$ vs. $17 / 21(P=0.13)$, stage 3 according ISS $11 / 28$ (39\%) vs $15 / 38(39 \%) \quad(P=1.00)$. Cytogenetic favourable aberrations in $10 / 28(36 \%)$ vs. $13 / 38(34 \%), P=0.61$ and unfavourable findings in $7 / 28(25 \%)$ vs. $10 / 38(26 \%)(P=1.00)$. Median TFS was 10 months vs. 18 months $(P=0.04)$ and median OS was not yet reached vs, 57 months $(P=0.005)$. At the dividing of second group in two subgroups - "low level of 
paraprotein" in time of Tx $(n=20)$ and "high level" in time of Tx $(n=18)$. Median TFS in subgroup ...high" are 4 months vs. 11 months $(P=0.0016)$, OS 44 months vs. 65 months $(P=0.03)$. Conclusion: We compared the files of tandem Tx and Tx in relapse in comparable groups of patients (age, cytogenetics, inductive input mode). From our results is evident favourable effect of tandem Tx on the TFS as on OS. In patients in relapse are clearly better TFS and OS in patients with lower baseline of paraprotein than in rapid relapse.

Disclosure of Interest: None declared.

AB93

Autologous stem cell transplantation in older multiple myeloma patients is safe and effective - a single centre experience

A. F. Baptista ${ }^{1, *}$, C. Geraldes ${ }^{2}$, R. Tenreiro ${ }^{1}$, A. Roque ${ }^{1}$ R. Guilherme', P. Cesar', S. Ramos', E. Cortesão ${ }^{2}$, A. I. Espadana ${ }^{2}$ M. L. Ribeiro ${ }^{2}$

${ }^{1}$ Centro Hospitalar e Universitário de Coimbra, Coimbra, Portugal, ${ }^{2}$ Centro Hospitalar e Universitário de Coimbra, Centro Hospitalar e Universitário de Coimbra, Coimbra, Portugal

Introduction: At the present time multiple myeloma (MM) is a consensual indication for high dose chemotherapy (HDC) followed by autologous stem cell transplantation (ASCT). However, most of the larger trials evaluated the role of ASCT in MM patients with 65 years of age $(Y)$ or younger. Once MM is frequently diagnosed in the elderly, it is crucial to understand if there is a role for ASCT in an older group of patients. In this study we aimed to compare the immediate toxicity and long term results of MM patients submitted to ASCT according to their age.

Material (or patients) and methods: We retrospectively evaluated all MM patients eligible to HDC and ASCT in our centre between January/2007 and December/2014. Analyzed data: age at the time of ASCT, number of hospitalization days (HD), number of days with neutropenia $<0,5 \times 10^{9} / \mathrm{L}$, number of days with thrombocytopenia $<20 \times 10^{9} / \mathrm{L}$, gastro-intestinal toxicity, oral mucositis, need for endovenous antibiotherapy, number of packed red blood cells (RBC) and platelet's concentrate transfusions, need for parenteral nutrition, treatment related mortality (TRM), progression free survival (PFS) and overall survival (OS). For the purpose of toxicity analysis, all the transplants were considered $(n=161)$ whereas for the outcome investigation, only first transplants were analyzed $(n=135)$.

Results: The male:female ratio was 1.3. At the time of the ASCT the median age was 59Y [28-70]. 132 patients were $<65 Y$ and $29 \geq 65 Y$. The elderly group had higher number of HD (mean 27 days vs 20 days; $P=0.0024$ ). The number of days with thrombocytopenia <20×109/L (8 Vs 5 days) and with neutropenia ( 7 Vs 9 days) and the number of RBC units transfused (1.4 Vs 2.5) were superior in the older group as well $(P=0.0025,0.0339$ and $P<0.0016$, respectively). There were no significant differences regarding other toxicity related parameters and the TRM was null in both groups. Regarding long term outcomes, PFS was similar in both groups (median PFS was $3.8 Y$ in the $<65 Y$ group and 4.0 in the $\geq 65 Y$, $P=0.35$ ). We did not find statistically significant differences in OS as well (median OS not reached in both groups, $P=0.17$ ). Conclusion: Despite the higher HD and hematological toxicity in the $\geq 65 \mathrm{Y}$ group, there was no major impact in patients' prognosis and also no increase in TRM. On the other hand, the outcome was comparable in both groups. Even aware of the limitations regarding the small sample size and the retrospective analysis, our experience indicates that ACST is safe and effective in $\mathrm{MM}$ patients older than $65 \mathrm{Y}$ as in the younger group.

Disclosure of Interest: None declared.
AB94

Are timing and quality of response predictors of asct outcome in multiple myeloma patients? - a single centre experience

A. F. Baptista ${ }^{1, *}$, C. Geraldes ${ }^{2}$, R. Tenreiro ${ }^{1}$, A. Roque ${ }^{1}$ R. Guilherme ${ }^{1}$, P. Cesar', S. Ramos ${ }^{2}$, E. Cortesão ${ }^{2}$, A. I. Espadana ${ }^{2}$ M. L. Ribeiro ${ }^{2}$

${ }^{1}$ Centro Hospitalar e Universitário de Coimbra, Coimbra, Portugal, ${ }^{2}$ Centro Hospitalar e Universitário de Coimbra, Centro Hospitalar e Universitário de Coimbra, Coimbra, Portugal

Introduction: Autologous stem cell transplantation (ASCT) remains a standard of care for multiple myeloma (MM) patients who are eligible to receive high-dose therapy. Factors that can predict success of this approach are still object of various studies. We aimed to investigate if early ASCT or better response to this therapy were predictive of treatment success. Material (or patients) and methods: We retrospectively reviewed the outcome of first ASCT in MM patients from 20072014. All tandem transplants were excluded. Groups analyzed: 1) patients receiving ASCT less than 1 year $(\mathrm{Y})$ after diagnosis $(<1 \mathrm{Y})$ Vs the ones who received ASCT $\geq 1 Y$ after and 2) patients who reached very good partial response or better ( $\geq$ VGPR) after ASCT Vs the ones with inferior responses $(<\mathrm{VGPR})$. Responses were evaluated 100 days after ASCT according to the International Myeloma Working Group response criteria.

Results: There were 135 patients with a median age at the time of the transplant of 58Y [28-69] and male:female ratio of 1.3. In 68 cases ASCT took place $<1 \mathrm{Y}$ and in $64 \geq 1 \mathrm{Y}$. Three patients were excluded from this analysis due to lack of clear information. We found that 74 reached very good parcial response or better ( $\geq$ VGPR) and 61 inferior responses ( $<$ VGPR). Median progression free survival (PFS) was superior in the early ASCT group (4.0Y Vs 1.5Y; $P=0.0021$ ). Overall survival (OS) was also superior in the $<1 Y$ ASCT (not reached Vs $4 Y, P=0.0112$ ). Median PFS was higher in the $\geq$ VGPR group (4.5Y Vs $2.8 \mathrm{Y}, P=0.0196)$. OS was slightly better in the $\geq$ VGPR group but there was no statistically significant difference (median OS not reached in any group; $P=0.161$ ).

Conclusion: Both timing of transplantation and quality of treatment responses seemed to be good predictors of clinical outcomes. Early transplantation associated with better PFS and OS. Moreover, the better response group showed higher PFS and OS, even though only superiority of PFS was proven statistically significant. Although being a small and retrospective study with considerable limitations, our experience favors the effort to proceed to ASCT as soon as possible and to achieve better responses in order to obtain a more effective control of the disease.

Disclosure of Interest: None declared.

\section{AB95}

Treatment of bence-jones multiple myeloma

with chemotherapy with/without stem cell

transplantation-treatment of bence-jones multiple myeloma with chemotherapy with/without stem cell transplantation-single centre experience

A. Zivanovic-Ivic ${ }^{1}$, D. Stamatovic ${ }^{1}$, O. Tarabar ${ }^{1}$, L. Atanaskovic ${ }^{1}$ M. Elez ${ }^{1, *}$, S. Marjanovic ${ }^{1}$, Z. Tatomirovic ${ }^{2}$, O. Radic-Tasic ${ }^{3}$, B. Balint ${ }^{4}$, G. Ostojic ${ }^{4}$, Z. Mijuskovic ${ }^{5}$, L. Tukic $^{\prime}$

${ }^{1}$ Military medical academy, Clinic of haematology, ${ }^{2}$ Military medical academy, ${ }^{3}$ Institut of pathology, ${ }^{4}$ Military medical academy, Institut of transfuziology, ${ }^{5}$ Military medical academy, Institut of medical biochemistry, Belgrade, Serbia

Introduction: Bence-Jones myeloma multiplex is a progressive disease characterized by excesssive numbers of abnormal plasma cells in the bone marrow and overproduction of incomplete immunoglobulins, containing only the light chain portion of the immunoglobulini. This type of myeloma occurs $15-20 \%$. The median overall survival is approximately 4 years. Pts outcome in BenceJones myeloma has been remarkably 
improved due to the use of combination therapies including chemotherapy and stem cell transplantation.

Material (or patients) and methods: A retrospective analysis of outcome of treatment Bence-Jones myeloma. Since 1995. until 2013. we treated 60 pts ( 39 men and 21 female), average age 55,5 years.ISS1 15 pts, ISS2 10 pts, ISS3 35 pts.Renal insufficiency was present in 35 pts. High risik pts was defined by the presence of following factors:b2M $>5,5 \mathrm{mg} / \mathrm{l}$, albumine $<3,5 \mathrm{gr} / \mathrm{dl}$, CRP $>6$, high lactat dehydrogenase, renal failure,stage III.Pts treated with induction,consolidation and maintenance therapy.Conventional induction treatments were applied as following regimens: $\operatorname{VAD}(42), M P(9), C T D(3), P A D(2), T A D(4)$

Results: Clinical response was achieved in 41 pts $(70 \%)$, while in 19 pts (30\%) established disease was resistant. SCT had been done with 33 pts (55\%), while 27 pts $(45 \%)$ were treated with conventional chemotherapy adjusted to the vital age and comorbiditity. In the group of pts with transplantation done tandem had been carried out with 11 pts and secondary SCT had been done in 4 relapsed pts. With 1 pts with tandem SCT allogenic (singen) SCT had been done. TRM is 3\%. Maintenance therapy had been done in 31 pts.Impact of high risk factors on outcome/PFS/OS was of no significance, except for elevated high lactat dehydrogenase values that are associated with rapid relapse. The presence of bone lesions associated with short PFS and with no difference in OS. In the group of transplanted no difference in OS with/without a response to primary therapy, but in the nontransplanted pts OS was significance shorter in patients who did not achieve a response.The transplanted patients had significantly longer PFS (mediana 14 months vs 8 months, $P<0,05$ ) and longer OS (mediana 50 months vs 20 months, $P<0,001) .20$ pts $(33 \%)$ of treated pts are living, while 40 pts $(67 \%)$ died. Univariate log. regres. analysis showed that non-transplant patients are 10,41 times more likely to terminate lethal compared to transplant patients (RR 10,41(95\%C.I.43,47-2,52), $P<0,001$ ).

Conclusion: Our study showed ASCT is a more effective method of treatment of patients with BenceJones myeloma compared to the conventional chemotherapy, but the results are still unsatisfactory. Impact of high risk factors on outcome/ PFS/OS was of no significance, except for elevated high lactat dehydrogenase values that are associated with rapid relapse. One of the major efforts to improve the results of intensive therapy and ASCT involves the integration of novel agents (proteasome inhibitors and immunomodulatory drugs) into the transplantation sequence.

Disclosure of Interest: None declared.

\section{AB96 \\ The outcome of bortezomib-based regimen for the first time induction chemotherapy in patients with multiple myeloma after auto SCT

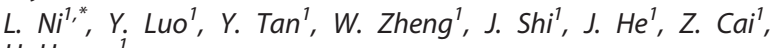 H. Huang ${ }^{i}$ \\ ${ }^{1}$ Bone Marrow Transplantation Center, The First Affiliated Hospital, Zhejiang University School of Medicine, hangzhou, China}

Introduction: The outcome of multiple myeloma (MM) has been significantly changed by novel agents. Bortezomib, as the representative, has been recommended as first-line treatment in guideline for many years. But whether bortezomib-based regimen as first induction chemotherapy has different outcome remains unknown.

Material (or patients) and methods: We retrospectively analyzed 38 patients with MM undergoing autologous stemcell transplantation to compare different outcomes in patients treated with bortezomib-based regimen $(n=25)$ for the first time induction chemotherapy to those without $(n=13$, including 9 cases administrating bortezomib later because of ineffectiveness of original plans or relapse) after newly diagnosed between 2007and 2014.

Results: Before transplantation, all patients achieved at least partial response (PR) according to International Myeloma
Working Group uniform response criteria. The median (range) numbers of peripheral blood mononuclear cells and $\mathrm{CD}_{3}{ }^{+}$ cells in each group were 9.43 and $10.12 \times 10^{8} / \mathrm{kg}(P=0.59), 5.02$ and $5.28 \times 10^{6} / \mathrm{kg}(P=0.87)$, respectively. The median (range) time to reach a neutrophil count $>0.5 \times 10^{9} / \mathrm{L}$ was $14(12.5-15)$ and $13(12-14)$ days $(P=0.89)$, respectively. Meanwhile, Platelets ( $>20 \times 10^{9} / \mathrm{L}$ without transfusion lasts for 7 days) recovered in average time of 15.5(12.25-20.75) and 14 (14-16) days $(P=0.17)$, respectively. The median overall survival (OS) after transplantation had not been reached in bortezomib-based group, and 54months in group without bortezomib. 1-year OS and 3 -year OS showed no difference ( $96 \%$ vs $85 \% ; 78 \%$ vs $75 \%, P=0.3$, respectively). Median time to disease progression in two groups was both 43 months.

Conclusion: Bortezomib-based regimen as the first induction chemotherapy may have no significant benefit in terms of OS and TTP. But further prospective randomized trials and multicenter researches are still required to verify whether this regimen make sense or not.

Disclosure of Interest: None declared.

\section{AB97}

Allogeneic hematopoietic stem cell transplantation in patients with relapsed or refractory multiple myeloma: possible cures and acceptable toxicities

M. M. M. Greco ${ }^{1, *}$, A. M. Carella ${ }^{1}$, E. Merla ${ }^{1}$, A. Falcone ${ }^{1}$, R. Angarano $^{1}$, N. Cascavilla

${ }^{1}$ Haematology, Ircss Casa Sollievo Della Sofferenza, San Giovanni Rotondo, Italy

Introduction: Although novel agents are providing uncontested benefits, multiple myeloma (MM) still remains an incurable malignancy. Allogeneic haematopoietic transplantation (allo-HSCT) may be a therapeutic option because of the inability of current therapies to eradicate myeloma cells.

Material (or patients) and methods: Our study retrospectively evaluated a distinct group of patients with relapsed/refractory multiple myeloma who underwent an allo-HSCT after 1-3 lines of treatment addressed to reduce the burden disease. Between January 2001 and December 2013, 30 relapsed/refractory patients (16 $\mathrm{M}$ and $14 \mathrm{~F}$, median age 52 ) received an allo-HSCT in advanced disease. Median age at start of diagnosis was 49 years, median age at time of allo-HSCT was 51 years. In pts with previous auto-HSCT the median interval from auto- to allo-HSCT was 15 months. Indications for allo-HSCT were: -refractory disease (response less than PR) after 2 lines of induction therapy (6 pts); -relapse or progression disease after single or double auto-HSCT (24 pts). The conditioning regimen was IC in 25 pts and MAC in 5 pts. Disease status at the time of transplant was partial response (PR) after 2-3 lines of treatment except of auto-HSCT in 24 pts $(80 \%)$ and refractory disease in 6 pts (20\%) The hematopoietic cell donors were 20 siblings and 10 URDs.

Results: Overall, 15 pts (55\%) achieved a CR; 8 pts (30\%) had a PR and 4 pts (15\%) showed a persistent refractory disease. 5 pts in CR (19\%) relapsed after the transplantation. The cumulative incidence of acute GVHD (aGVHD) grade II-IV was $27 \%$. About half pts developed chronic graft versus host disease (cGVHD), with an equal frequency as limited or extensive and a cumulative incidence of $52 \%$. TRM before day $100^{\text {th }}$ regarded 2 cases due to aGVHD grade IV. NRM was $17 \%$. 2 pts in CR with extensive CGVHD died because of obliterans bronchiolitis at +60 and +32 months respectively. Interestingly, 2 additional pts in CR with oral CGVHD incurred a fatal tongue neoplasia and a non-fatal intestinal neoplasia. The relapse and/or progression of disease were observed in 14 pts $(51 \%)$ and resulted the cause of death in 12 pts (44\%). A total of $10(33 \%)$ patients are still alive and $8(26 \%)$ pts are in a complete remission state. The median time of follow-up was 54 months. TRM at 1 and 5 years was respectively $6 \%$ and $19 \%$. OS at five years was $51 \%$. PFS at 3 years was $45 \%$, while nearly $25 \%$ at 6 years. Disease status prior to allo-HSCT and cGVHD was significantly associated with the risk of mortality, with limited cGVHD resulting protective. 
Conclusion: The role of allo-HSCT in multiple myeloma is strongly discussed and frequently neglected. Due to of small numbers, heterogeneity of patients and follow-up from periods with different therapeutic protocols, there is no conclusive consensus of allo-HSCT implementation. Our retrospective analysis suggests that in the setting of primary refractory disease allo-HSCT is probably ineffective. However, in the contest of responsive therapy disease allo-HSCT could determine $C R$ and possibly offer permanent cures.The current use of new agents can determine a more incisive reduction of burden disease before allo-HSCT trough increasing its efficacy. Disclosure of Interest: None declared.

\section{AB98}

The relation between initial 18F-FDG PET/CT findings and baseline clinical parameters in multiple myeloma

T. Tuglular ${ }^{1, *}$, F. Pepedil Tanrikulu' ${ }^{1}$, T. Toptas ${ }^{1}$, A. Karadeniz ${ }^{1}$ A. Eser ${ }^{1}$, O. Kara ${ }^{1}$, I. Atagunduz

${ }^{1}$ Marmara University Hospital, Istanbul, Turkey

Introduction: In a recent study, a positive correlation was reported between baseline fluorodeoxyglucose (FDG) positron emission tomography (PET) findings and clinical parameters in patients with multiple myeloma (MM). FDG-PET findings were associated with disease outcomes.

Material (or patients) and methods: We aimed to investigate the correlation between baseline FDG-PET findings and clinical parameters in our MM patients. We conducted a retrospective analysis in a total of 385 patients that were followed up between January 2005 and December 2013. Forty-eight chemotherapy naïve patients were found to have baseline FDG-PET evaluation. We compared the initial clinical characteristics of these patients with their PET findings: the number of focal bone lesions (FLs) and the maximum standardized uptake value (SUVmax).

Results: Twenty-three out of 48 patients were male. The median age was 62 years. Among patients with secretory disease (97.9\%), $\lg A, \lg G$, and light chain only disease were evident in $8.3 \%$, $62.5 \%$, and $27.1 \%$, respectively. The median percentage of bone marrow plasma cells was $40 \%$ (range $25-60$ ). Median serum beta2 microglobulin was $3.5 \mathrm{mg} / \mathrm{L}$ (range: $2.5-5.0$ ) and $20 \%$ of patients had international staging system (ISS) stage 3. Durie-Salmon (DS) stage III disease was detected in $43.8 \%$. Forty-one $(85.4 \%)$ had bone lesions on FDG-PET imaging. Of those, 17 (54.8\%) had more than three FLs. The median SUVmax for those FLs was 6.5 (range: 4.9-10). The number of FLs on PET correlated only with DS stage III disease.

Conclusion: A statistically significant positive correlation was found between baseline FDG-PET findings and DS stage in MM patients.

Disclosure of Interest: None declared.
AB99

Outcome of $\mathbf{2 9}$ myeloablative allogenic hematopoietic cell transplantations in multiple myeloma

Z. Csukly, ${ }^{1,}$, A. Barta ${ }^{1}$, A. Batai ${ }^{1}$, L. Gopcsa ${ }^{1}$, L. Lengyel ${ }^{1}$, P. Remenyi ${ }^{1}$, M. Reti ${ }^{1}$, E. Torbagyi ${ }^{1}$, T. Masszi ${ }^{1}$

${ }^{1}$ Dept. of Haematology and stem cell transplantation, St. Laszlo Hospital, Budapest, Hungary

Introduction: The purpose of this study was to assess prognostic factors which determine overall survival (OS) and progression free survival (PFS) of patients with multiple myeloma who received myeloablative conditioning and allogenic stem-cell transplantation (allo-SCT).

Material (or patients) and methods: Data were reviewed of 28 consecutive patients who received allo-SCT between 2007 and 2015. Patients caracteristics was 19 men and 9 women, median age at allo-SCT 47 years (28-58). The median number of therapies before allo-SCT was 4 (2-7 ).Most of the patients $(26 / 28)$ received high dose melphalan and autologous stem cell transplantation before allo-SCT. Tumor status at allo-SCT: complett remission (CR) $n=5(18 \%)$, very good partial remission (VGPR) $n=3(11 \%)$, partial remission (PR) $n=11$ (39\%), progressive disease (PD) $n=9(32 \%)$. Median time from diagnosis to allo-SCT 35 month (12-148), conditioning: total body irradiation (12 Gy) /melphalan $(140 \mathrm{mg} / \mathrm{m} 2) \quad n=22$ (79\%), busulphan $(16 \mathrm{mg} / \mathrm{kg}) / \mathrm{melphalan}(140 \mathrm{mg} / \mathrm{m} 2) n=6$ (21\%), donor type: HLA identical sibling $n=15(54 \%)$, matched unrelated (MUD) $n=6(21 \%)$, mismatched unrelated $n=7$ (25\%), median follow up from allo-SCT 10,1 month (0,3-95,8). All the patients received tacrolimus/sirolimus GVHD profilaxis. Results: One-year and three-year OS/PFS was 44/44\% and $28 / 20 \%$. One-year and three-year PFS in the CR group was $100 / 67 \%$, in the VGPR $50 / 50 \%$, in the PR $40 / 20 \%$ and in the PD $12,5 / 0 \%$. Transplantation related mortality (TRM) was $42,8 \%$, in the CR group 0\%, in the VGPR 33\%, in the PR 60\% and in the PD $62,5 \%$. Acut GVHD was $62 \%$ (grade I-II 55\%, III-IV 45\%). TRM in the acut GVHD group was $67 \%$. Chronic GVHD was $28 \%$. The incidence of CGVHD among the three-year survivors was $42 \%$. Two patients was given DLI during of progression or disease relapse without effectivity. There was no significant difference neither in OS nor in PFS depending from the donor type, conditioning therapy, time from the diagnosis to allo-SCT, age (under/above 47 years), cytogenetic abnormalities and number of therapies before allo-SCT.

Conclusion: TRM is very frequent in PR and PD groups. In CR group TRM is zero so it seems to be important to treat the patients before allo-SCT until CR or VGPR. Allo-SCT is useless for the patients with PD. Chronic limited GVHD is necessary for long survival. DLI treatment could be given for patients with residual disease after allo-SCT routinely. In the low residual tumor groups (CR, VGPR) the myeloablative allo-SCT can be curative.

Disclosure of Interest: None declared.

\begin{tabular}{|c|c|c|c|c|c|c|}
\hline \multirow[b]{2}{*}{ Variables } & \multicolumn{4}{|c|}{ Number of focal bone lesions $>3$} & \multicolumn{2}{|c|}{ SUVmax $\geq 6.5$} \\
\hline & OR & $95 \% \mathrm{Cl}$ & $\mathbf{P}$ & OR & $95 \% \mathrm{Cl}$ & $\mathbf{P}$ \\
\hline Age at diagnosis $>62$ years & 0.57 & $0.09-13.89$ & 0.71 & 1.18 & $0.23-6.20$ & 1.00 \\
\hline Male gender & 1.74 & $0.32-10.75$ & 0.71 & 1.12 & $0.22-5.78$ & 1.00 \\
\hline $\lg A$ & 0.16 & $0-2.12$ & 0.16 & 0.32 & $0-4.31$ & 0.39 \\
\hline Lambda light chain & 0.28 & $0.04-1.71$ & 0.20 & 0.42 & $0.07-2.44$ & 0.45 \\
\hline ISS, stage II or III & 1.33 & $0.14-12.05$ & 1.00 & 2.22 & $0.23-24.48$ & 0.70 \\
\hline Durie-Salmon stage III & 7.48 & $1.13-88.55$ & 0.03 & 0.84 & $0.16-4.34$ & 1.00 \\
\hline Serum creatinine $\geq 2.0 \mathrm{mg} / \mathrm{dL}$. & 1.34 & $0.09-78.64$ & 1.00 & 0.26 & $0.004-3.68$ & 0.50 \\
\hline Hemoglobin $<10 \mathrm{~g} / \mathrm{dL}$ & 0.31 & $0.03-2.01$ & 0.31 & 0.80 & $0.14-4.25$ & 1.00 \\
\hline Corrected serum calcium $>11 \mathrm{mg} / \mathrm{dL}$ & 1.96 & $0.16-109.28$ & 0.99 & 0.58 & $0.04-6.01$ & 0.93 \\
\hline Serum albumin $<3.5 \mathrm{~g} / \mathrm{dL}$ & 0.14 & $0.003-1.38$ & 0.12 & 0.31 & $0.04-1.85$ & 0.26 \\
\hline Serum beta- 2 microglobulin $>3.5 \mathrm{mg} / \mathrm{L}$ & 0.27 & $0.02-2.47$ & 0.35 & 3.46 & $0.34-54.32$ & 0.44 \\
\hline Serum $\mathrm{CRP}>4 \mathrm{mg} / \mathrm{dL}$. & 1.00 & $0.10-10.33$ & 1.00 & 1.52 & $0.17-14.48$ & 1.00 \\
\hline Bone marrow plasma cells $>30 \%$ & 1.57 & $0.23-10.27$ & 0.86 & 1.41 & $0.23-9.01$ & 0.96 \\
\hline
\end{tabular}


AB100

Distribution Patterns of Angiogenesis in Colorectal Cancer: Study Protein Expression of CD105 (Endoglin)

R. Minhajat ${ }^{1,2, *}$, D. Bayu ${ }^{2}$, A. F. Benyamin ${ }^{2}$, U. A. Miskad ${ }^{3}$, T. Harjianti $i^{2}$

${ }^{1}$ Histology, ${ }^{2}$ Hematology and Medical Oncology Division, Internal Medicine Department, 3 Pathology Department, Medical Faculty Hasanuddin University, Makassar, Indonesia

Introduction: Angiogenesis plays an important role in the growth and metastasis of colorectal carcinoma, which is currently one of the targets of cancer therapy. It has been reported that the CD105 (Endoglin) involved in angiogenesis and is a powerful marker for angiogenesis in colorectal carcinoma. Level Quantitative angiogenesis in peritumor and intratumor area is important to know because it is closely related to the micro environmental factors that influence the occurrence of cancer angiogenesis. The goal of this study analyze the distribution pattern of angiogenesis in colorectal cancer by comparing the distribution of angiogenesis in peritumor and intratumor areas between well, moderate and poorly differentiated colorectal carcinoma, and between carcinoma with metastasis and nonmetastasis.
Material (or patients) and methods: This study analysed fifty samples of resected colorectal adenocarcinoma. Angiogenesis was assessed by immunohistochemical method using a primary monoclonal antibody CD105. Positive expression of CD105 was assessed through the CD105 protein expression in vascular endothelial cells, while the distribution pattern of angiogenesis assessed by counting the positive expression of CD105 protein in hot spots by using the MVD (microvessel density) in the peritumor and intratumor areas and then performed statistical analysis.

Results: There is a significant difference between quantitative level of angiogenesis in peritumor and intaratumor areas of well $(P<0.01)$, moderate $(P<0.01)$ and poorly $(<0.05)$ differentiated adenocarcinoma. Significant difference between the quantitative levels of angiogenesis in peritumor and intratumor areas of non-metastatic colorectal cancer $(P<0.01)$ and lymph node metastases $(<0.05)$ was found, but not in colorectal cancer with liver metastatis.

Conclusion: The results of this study provide a fundamental data regarding the distribution patterns of angiogenesis in colorectal carcinoma that may be beneficial in development strategies of antiangiogenesis.

Disclosure of Interest: None declared. 